\title{
Espaços finitos de probabilidade
}

\author{
Daniel Polacchini Octaviano
}



Data de Depósito:

Assinatura:

\title{
Daniel Polacchini Octaviano
}

\section{Espaços finitos de probabilidade}

\begin{abstract}
Dissertação apresentada ao Instituto de Ciências Matemáticas e de Computação - ICMC-USP, como parte dos requisitos para obtenção do título de Mestre em Ciências - Programa de Mestrado Profissional em Matemática. EXEMPLAR DE DEFESA.
\end{abstract}

Área de Concentração: Matemática

Orientador: Prof. Dr. Hermano de Souza Ribeiro

\section{USP - São Carlos}

Agosto de 2015 
Ficha catalográfica elaborada pela Biblioteca Prof. Achille Bassi e Seção Técnica de Informática, ICMC/USP, com os dados fornecidos pelo(a) autor(a)

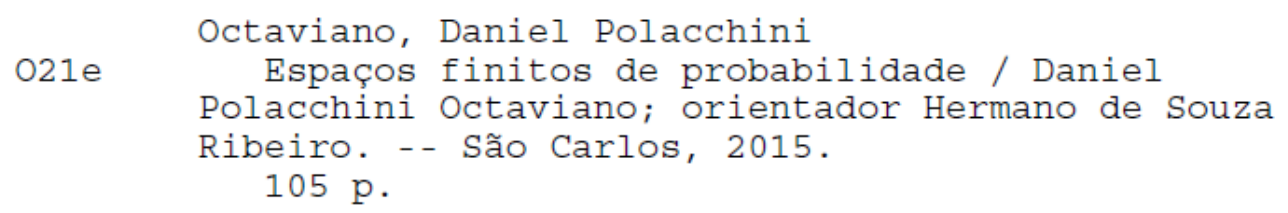

1. Probabilidades . 2. Princípio da inclusãoexclusão. 3. Teorema de Bayes. 4. Ensino da matemática. I. Ribeiro, Hermano de Souza, orient. II. Título. 


\section{Daniel Polacchini Octaviano}

\section{Finite probability spaces}

Master dissertation submitted to the Instituto de Ciências Matemáticas e de Computação - ICMCUSP, in partial fulfillment of the requirements for the degree of Mathematics Professional Master's Program. EXAMINATION BOARD PRESENTATION COPY.

Concentration Area: Mathematics

Advisor: Prof. Dr. Hermano de Souza Ribeiro

USP - São Carlos

August 2015 

Dedico este trabalho a toda minha família, mas em especial aos meus pais Clodoaldo e Ezilia que sempre me incentivaram com muito amor e carinho. 


\section{Agradecimentos}

Primeiramente agradeço a Deus por ter me dado saúde e paz durante a realização deste trabalho.

À minha noiva Marcela pelo apoio, amizade, carinho e amor que sempre me dedicou.

Ao meu professor orientador Prof. Dr. Hermano de Souza Ribeiro pelo apoio, incentivo e amizade.

À toda minha família, em especial aos meus irmãos, cunhadas e sobrinhos, pelo incentivo e por fazerem parte da minha vida. 


\section{Resumo}

Este trabalho aborda aspectos básicos do cálculo de probabilidades em espaço finito de probabilidade, aplicados a eventos como lançamentos de moedas, jogos de pôquer, loterias, problemas de nascimento de crianças e problemas com urnas, e tem como objetivo principal exibir, em cada fenômeno aleatório, o espaço finito de probabilidade que é o modelo probabilístico do fenômeno. Em cada exemplo de aplicação, foram definidos os espaços amostrais e a função de probabilidade correspondente, além do evento cuja probabilidade é a resposta do problema apresentado. A visualização concreta do espaço dos eventos associado a um processo aleatório facilita a compreensão do cálculo de probabilidades.

Palavras-chave: Probabilidade. Espaços finitos de probabilidades. Noções de probabilidades. 


\section{Abstract}

This dissertation approaches basic aspects of the probability calculus in finite probability space, applied to events such as coins tosses, poker matches, lotteries, birth problems and problems with voting machines, and it aims at displaying in each random phenomenon, the finite probability space which is the probability model of the phenomenon. In each working example, the sample spaces and their corresponding probability function have been defined, besides the event whose probability is the response of the problem presented. The concrete visualization of the space of events associated with a random process facilitates the understanding of the probability calculus.

Keywords: Probability. Finite probability spaces. Notions of probability. 


\section{Sumário}

\begin{tabular}{|lll}
1 & PROBABILIDADES - DEFINIÇÕES & 15
\end{tabular}

1.1 ANÁLISE COMBINATÓRIA $\ldots \ldots \ldots \ldots \ldots$

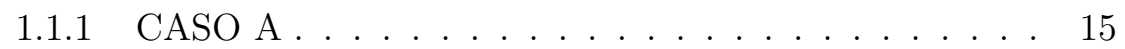

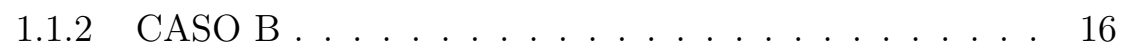

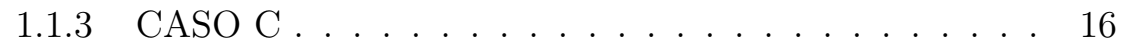

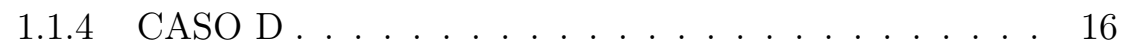

1.2 ESPAÇO FINITO DE PROBABILIDADE . . . . . . . . . . 18

1.3 ESPAÇO FINITO EQUIPROVÁVEL DE PROBABILIDADE 19

$\begin{array}{llll}1.4 & \text { ESPACCO DE PROBABILIDADE } \ldots \ldots \ldots & \ldots\end{array}$

1.5 NASCIMENTO DE FILHOS $\ldots \ldots \ldots \ldots \ldots$

1.6 LANÇAMENTO DE MOEDAS . . . . . . . . . . . . . . . . . 23

1.7 PROBLEMAS DE PROBABILIDADE COM DADOS . . . . 26

1.7 .1 LANÇAMENTO DE UM DADO . . . . . . . . . . . . 26

1.7.2 LANÇAMENTO SIMULTÂNEO DE DOIS DADOS

\begin{tabular}{|lll}
\hline & DISTINTOS $\ldots \ldots \ldots$. . . . . . . . . . . . . . . . & 27 \\
\hline 1.7 .3 & LANÇAMENTO SIMULTÂNEO DE CINCO DADOS \\
\hline
\end{tabular}

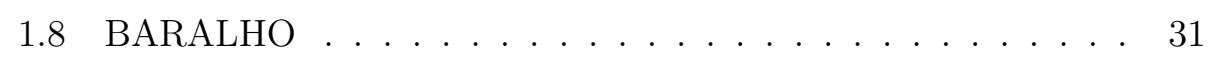

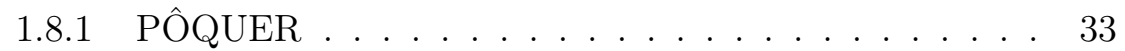

$1.8 .2 \quad$ PÔQUER COM DADOS $\ldots \ldots \ldots \ldots$

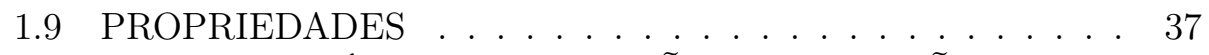

1.9.1 PRINCÍPIO DA INCLUSÃO E EXCLUSÃO PARA DOIS EVENTOS . . . . . . . . . . . . . . . . . . 39

1.9.2 PRINCÍPIO DA INCLUSÃO E EXCLUSÃO PARA TRÊS EVENTOS . . . . . . . . . . . . 40

1.9.3 PRINCÍPIO DA INCLUSÃO E EXCLUSÃO PARA N EVENTOS .................. 41

1.9 .4 TEOREMA DE WARING PARA DOIS EVENTOS . 43

1.9.5 TEOREMA DE WARING PARA TRÊS EVENTOS . 44 
1.9 .6 TEOREMA DE WARING . . . . . . . . . . . . . . . 45

1.9 .7 DESIGUALDADE DE BOOLE . . . . . . . . . . . . . 48

1.9 .8 DESIGUALDADE DE BONFERRONI . . . . . . . . 49

1.10 PROBABILIDADE CONDICIONAL . . . . . . . . . . 50

1.11 FÓRMULA SEQUENCIAL DE BAYES . . . . . . . . . 54

1.12 TEOREMA DE BAYES $\ldots \ldots \ldots \ldots \ldots \ldots$

1.13 TEOREMA DE BAYES PARA PROBABILIDADE CONDI-

CIONAL . . . . . . . . . . . . . . . . . . . . . . 59

1.14 EVENTOS INDEPENDENTES . . . . . . . . . . . . . . . . 63

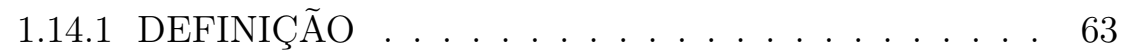

1.14 .2 PROPRIEDADES $\ldots \ldots \ldots$. . . . . . . . . . . . 64

1.15 ESPAÇO PRODUTO CARTESIANO DE DOIS EPAÇOS DE

PROBABILIDADE . . . . . . . . . . . . . . . . . 72

1.16 PROBABILIDADES BINOMIAIS . . . . . . . . . . 73

1.16.1 TEOREMA DAS PROBABILIDADES BINOMIAIS . 73

1.16.2 TEOREMA DAS PROBABILIDADES MULTINOMI-

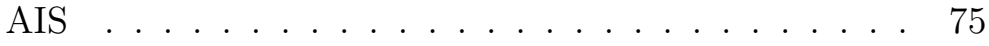

1.16.3 TEOREMA DAS PROBABILIDADES BINOMIAIS

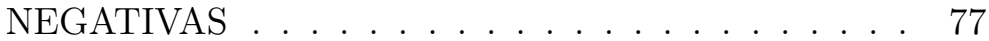

1.17 PROBLEMAS DE PROBABILIDADE COM URNAS . . . 78

1.18 UM PROBLEMA COM N URNAS . . . . . . . . . . . . . . 84

1.18 .1 AMOSTRAS NÃO ORDENADAS . . . . . . . . . 85

1.19 CÁLCULO DE PROBABILIDADE DE AMOSTRAS ORDE-

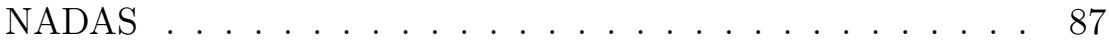

1.19.1 CASO A - Cálculo de probabilidade de amostras or-

\begin{tabular}{|l|}
\hline denadas com reposição de elementos de um conjunto \\
\hline \hline não vazio $\ldots \ldots \ldots \ldots \ldots \ldots \ldots \ldots \ldots \ldots \ldots$ \\
\hline
\end{tabular}

1.19.2 CASO B - Cálculo de probabilidade de amostras ordenadas sem reposição de elementos de um conjunto não vazio . . . . . . . . . . . . . . . . . . 88

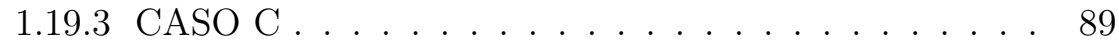

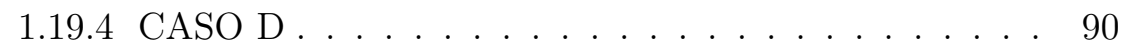

1.19 .5 CASO E . . . . . . . . . . . . . . . . . . . . 90

1.19 .6 CASO F $\ldots \ldots \ldots \ldots \ldots$

1.20 CÁLCULO DE PROBABILIDADE DE AMOSTRAS NÃO ORDENADAS . . . . . . . . . . . . . . . 91

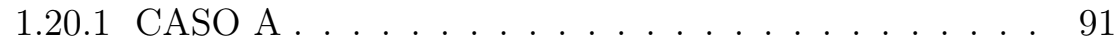

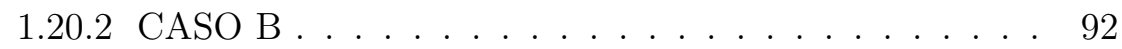

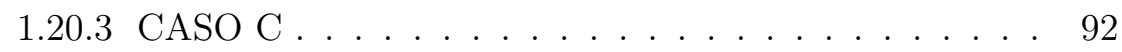

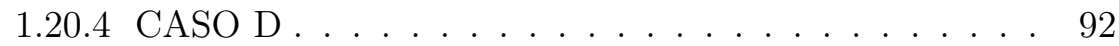




\subsection{PROBLEMAS DE OCUPAÇÃO EM URNAS COM BOLAS}

IDÊNTICAS $\ldots \ldots \ldots \ldots \ldots . \ldots \ldots$

1.21 .1 CASO A $\ldots \ldots \ldots \ldots$

1.21 .2 CASO B $\ldots \ldots \ldots \ldots . \ldots \ldots$

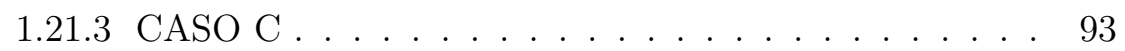

1.21 .4 CASO D . . . . . . . . . . . . . . . . . 95

1.22 PROBLEMAS DE OCUPAÇÃOO EM URNAS COM BOLAS

IDÊNTICAS OU COM BOLAS NUMERADAS . . . . . . . . 95

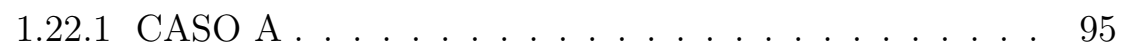

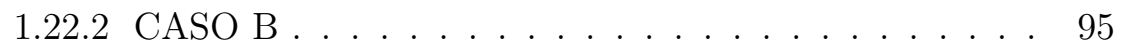

1.22 .3 CASO C $\ldots \ldots \ldots \ldots \ldots$

1.22 .4 CASO D $\ldots \ldots \ldots \ldots$

1.22 .5 CASO E . . . . . . . . . . . . . . . . 96

1.23 PROBLEMAS DE OCUPAÇÃO EM URNAS COM BOLAS

NUMERADAS . . . . . . . . . . . . . . . . . . . . 97

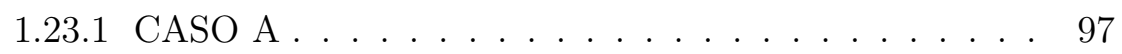

1.23 .2 CASO B . . . . . . . . . . . . . . . . . . . . 97

1.23 .3 CASO C $\ldots \ldots \ldots \ldots$

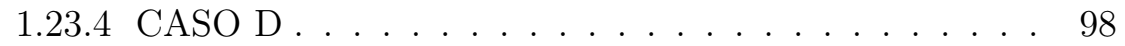

\begin{tabular}{l}
1.24 LOTERIAS $\ldots \ldots \ldots$ \\
\hline
\end{tabular}

1.24 .1 MEGA-SENA . . . . . . . . . . . . . . . . . . . . . . 102 


\section{Introdução}

A teoria matemática de probabilidade tem como objetivo a análise de processos aleatórios que são processos, cujos resultados não podem ser determinados a priori antes da ocorrência dos mesmos, mas o resultado de um processo aleatório é um de vários resultados possíveis.

O espaço amostral do experimento aleatório é constituído por todos os resultados possíveis e, a cada resultado possível está associado uma probabilidade que é um número real compreendido entre zero e um.

A teoria da probabilidade tem início no século XVII quando o matemático francês Blaise Pascal considerou a probabilidade de por exemplo de obtenção do valor 1 ou 6 no lançamento de um dado e neste caso, o espaço amostral é constituído pelos seis valores $1,2,3,4,5,6$ possíveis e a probabilidade de obtenção de 1 ou 6 foi definida no século seguinte por Laplace como o número de resultados favoráveis 1 ou 6 dividido pelo número de valores possíveis 1 , $2,3,4,5,6$ ou seja $\frac{2}{6}$.

Os eventos associados a um experimento aleatório são subconjuntos do espaço amostral: no caso do exemplo acima $A=\{1,6\} \subset\{1,2,3,4,5,6\}$.

A dissertação trata de espaços finitos de probabilidade associados a processos aleatórios em que o número de resultados possíveis de processo é finito, isto é, o espaço amostral é constituído por um número finito de elementos.

Os espaços finitos equiprováveis de probabilidade estudados inicialmente na dissertação, são os espaços em que a definição de probabilidade é dada por Laplace e são os espaços associados a problemas com dados não viciados, moedas não viciadas, loterias e jogos honestos de cartas como pôquer e bridge.

Espaços finitos não equiprováveis também são apresentados no texto como é o caso de moedas viciadas em que a probabilidade de cara é o triplo da probabilidade de coroa. Quando uma moeda viciada é lançada três vezes e é observado que no primeiro lançamento ocorreu coroa, a probabilidade de que ocorra cara nos próximos dois lançamentos origina o conceito de probabilidade condicional e o conceito de eventos independentes os quais também 
são estudados na dissertação.

Os teoremas clássicos, o princípio da inclusão-exclusão, teorema de Bayes, de Waring, das probabilidades binomiais, das probabilidades multinomiais e das probabilidades binomiais negativas além das desigualdades de Boole e Bonferroni, embora válidas para espaços quaisquer de probabilidades, são apresentados em espaços finitos de probabilidade.

Problemas envolvendo uma ou várias urnas, exemplificam os resultados clássicos e problemas com reposição ou sem reposição em urnas em que as bolas das urnas são numeradas ou idênticas entre si são analisados resultando a diferença existente entre as amostras ordenadas e não ordenadas. Problemas de ocupação em urnas encerram a dissertação.

O objetivo principal da dissertação foi exibir, em cada fenômeno aleatório, o espaço finito de probabilidade que é o modelo probabilístico do fenômeno. Em cada exemplo de aplicação, foram definidos os espaços amostrais e a função de probabilidade correspondente, além do evento cuja probabilidade é a resposta do problema apresentado. A visualização concreta do espaço dos eventos associado a um processo aleatório facilita a compreensão do cálculo de probabilidades.

O tema da dissertação, além de estar presente no nosso cotidiano com os jogos de azar e as loterias da Caixa Econômica Federal, faz parte do currículo oficial da Secretaria de Educação do Estado de São Paulo referente ao $9^{\mathrm{o}}$ ano do Ensino Fundamental (Probabilidade: Problemas de Contagem e Introdução a Probabilidade) e a segunda série do Ensino Médio (Probabilidade Simples, Probabilidade da união e/ou intersecção de eventos, Probabilidade condicional, distribuição binomial de probabilidades) e é tema muito frequente em grandes vestibulares do país e no ENEM. 


\section{Capítulo 1}

\section{PROBABILIDADES - DEFINIÇÕES}

\subsection{ANÁLISE COMBINATÓRIA}

Abaixo seguem algumas técnicas de contagem, para que possamos utilizar posteriormente para o cálculo do número de elementos de eventos e também para o número de elementos dos espaços amostrais.

Seja um conjunto $U$ não vazio com $n$ elementos

\subsubsection{CASO A}

Para cada $k \in \mathbb{N}=\{1,2,3, \ldots\}$ existem $n^{k}$ sequências distintas com $k$ elementos $\left(u_{1}, u_{2}, \ldots, u_{n}\right)$ em que $u_{j} \in U$ para cada $j=1,2,3, \ldots, k$.

Exemplo 1.1.1. O número de respostas distintas de um teste com 10 questões do tipo verdadeiro ou falso é dado por $2^{10}$. O número de folhas distintas de resposta na primeira fase do vestibular da FUVEST, com 90 questões de multipla escolha e cinco alternativas cada questão é a $5^{90}$.

$\mathrm{O}$ cadastro de pessoas físicas $(\mathrm{CPF})$ é o registro de um cidadão na Receita Federal brasileira. O CPF é composto por 11 dígitos da forma abc.def.ghi.XY em que $X Y$ são dígitos verificadores, e além disso, o algarismo $i$ que antecede os dígitos verificadores indica o estado que o CPF foi emitido, por exemplo se o algarismo $i$ for igual a 6 significa que o CPF foi emitido no estado de Minas Gerais e se $i$ for igual a 8, o CPF foi emitido no estado de São Paulo, logo os números dos CPF emitidos pelo estado de São Paulo são da forma abc.def.gh8.XY, sendo assim seria possível a emitir $10^{8}$ cadastros de pessoas físicas. 


\subsubsection{CASO B}

Para cada $k \in \mathbb{N}=\{1,2,3, \ldots\}$ com $k \leq n$ existem $(n)_{k}=n \cdot(n-1) \cdot(n-$ $2) \cdot \ldots \cdot(n-k+1)$ sequências distintas com $k$ elementos $\left(u_{1}, u_{2}, \ldots, u_{n}\right)$ em que $u_{j} \in U$ para cada $j=1,2,3, \ldots, k$ e que $u_{i} \neq u_{j}$ para $i, j \in\{1,2,3, \ldots, k\}$ e $i \leq j$.

Exemplo 1.1.2. O número de modos diferentes que podem ser escolhidos um presidente, um vice presidente e um secretário entre os elementos de um conselho com 10 membros é dado por $(10)_{3}=10 \cdot 9 \cdot 8=720$.

$\mathrm{Na}$ final de uma prova de natação, 50 metros livres, na qual 8 atletas estão disputando medalhas de ouro, prata e bronze, há $(8)_{3}$ maneiras de ocorrer a premiação.

\subsubsection{CASO C}

Para cada $k \in \mathbb{N}=\{1,2,3, \ldots\}$ com $k \leq n$ existem $\left(\begin{array}{l}n \\ k\end{array}\right)=\frac{n !}{k ! \cdot(n-k) !}$ subconjuntos distintos de $U$ com $k$ elementos.

Exemplo 1.1.3. O número de maneiras distintas de escolhermos um grupo de três pessoas entre as 10 pessoas de uma sala é $\left(\begin{array}{c}10 \\ 3\end{array}\right)=120$.

O número de comissões formadas por dois professores e três estudantes de uma escola, escolhidos entre 5 professores e 10 estudantes é dado por

$$
\left(\begin{array}{l}
5 \\
2
\end{array}\right) \cdot\left(\begin{array}{c}
10 \\
3
\end{array}\right)
$$

\subsubsection{CASO D}

Para cada $k \in \mathbb{N}=\{1,2,3, \ldots\}$ e para cada sequência finita $n_{1}, n_{2}, \ldots, n_{k}$ de números inteiros não negativos com a propriedade $n_{1}+n_{2}+\ldots+n_{k}=n$, exitem $\frac{n !}{n_{1} ! n_{2} ! \ldots n_{k} !}$ partições distintas $\left(A_{1}, A_{2}, \ldots, A_{k}\right)$, isto é

$$
A_{1} \cup A_{2} \cup \ldots \cup A_{k}=U \text { e } A_{i} \cap A_{j}=\phi \text { para } i, j \in\{1,2, \ldots, k\} \text { com } i \neq j
$$

com a propriedade de que para cada $j \in\{1,2, \ldots, k\}$ o número $\left|A_{j}\right|$ de elementos do subconjunto $A_{j} \subset U$ é igual a $n_{j}$, isto é, $\left|A_{j}\right|=n_{j}$.

Demonstração. (d)

A escolha dos elementos do primeiro subconjunto $A_{1}$ da partição de $U$ com $\left|A_{1}\right|=n_{1}$ é feita de $\left(\begin{array}{c}n \\ n_{1}\end{array}\right)$ manieras distintas.

A escolha dos elementos do segundo subconjunto $A_{2}$ da partição de $U$ com 
$\left|A_{2}\right|=n_{2}$ é feita de $\left(\begin{array}{c}n-n_{1} \\ n_{2}\end{array}\right)$ manieras distintas.

Sendo assim, a escolha dos elementos do último subconjunto $A_{k}$ da partição de $U$ com $\left|A_{k}\right|=n_{k}$ é feita de $\left(\begin{array}{c}n-n_{1}-n_{2}-\ldots-n_{k-1} \\ n_{k}\end{array}\right)$ maneiras distintas.

O princípio multiplicativo da contagem implica que a escolha de uma partição de $U$ com $k$ subconjuntos de $U$ é igual ao produto das escolhas de $A_{1}, A_{2}, \ldots, A_{k}$ isto é,

$$
\frac{n !}{n_{1} ! n_{2} ! \ldots n_{k} !}
$$

Exemplo 1.1.4. O número de maneiras distintas de uma rodada de cartas de uma mesa de bridge (em que os jogadores são nomeados por Norte, Oeste, Sul e Leste) é igual a

$$
\left(\begin{array}{l}
52 \\
13
\end{array}\right) \cdot\left(\begin{array}{l}
39 \\
13
\end{array}\right) \cdot\left(\begin{array}{l}
26 \\
13
\end{array}\right) \cdot\left(\begin{array}{l}
13 \\
13
\end{array}\right)=\frac{52 !}{13 ! \cdot 13 ! \cdot 13 ! \cdot 13 !}
$$

cujo o valor é superior a $5 \cdot 10^{28}$ enquanto que o número de mãos distintas de 13 cartas de um jogador de uma mesa de bridge é igual a

$$
\left(\begin{array}{l}
52 \\
13
\end{array}\right)=\frac{52 !}{13 ! \cdot 39 !}
$$

cujo valor é superior a $6 \cdot 10^{11}$.

O mesmo raciocínio pode ser aplicado na distribuição de peças de um jogo de dominó, em que 28 peças são distribuídas para quatro jogadores também nomeados Norte, Oeste, Sul e Leste em que cada um dos jogadores recebe 7 peças cada um. O número de maneiras distintas de distribuir as 28 peças para os 4 jogadores é dada por

$$
\left(\begin{array}{c}
28 \\
7
\end{array}\right) \cdot\left(\begin{array}{c}
21 \\
7
\end{array}\right) \cdot\left(\begin{array}{c}
14 \\
7
\end{array}\right) \cdot\left(\begin{array}{l}
7 \\
7
\end{array}\right)=\frac{28 !}{7 ! \cdot 7 ! \cdot 7 ! \cdot 7 !} .
$$




\subsection{ESPAÇO FINITO DE PROBABILIDADE}

O conceito básico da teoria de probabilidades no caso finito é o de espaço finito de probabilidade ou modelo probabilístico finito $\left(\Omega, 2^{\Omega}, P\right)$, em que $\Omega$ é o conjunto de todos as possíveis amostras de um dado experimento aleatório denominado espaço amostral do experimento aleatório, $2^{\Omega}$ é o conjunto constituído por todos os subconjuntos de $\Omega$ denominado conjunto dos eventos de um experimento aleatório e $P$ é uma função que associa a cada evento $A$ do experimento uma probabilidade $P(A)$, que é um número real não negativo e menor ou igual a um, denominado função de probabilidade do experimento aleatório sujeita aos postulados de Kolmogorov explicitados nas seguintes definições.

Um espaço finito de probabilidade é uma terna ordenada $\left(\Omega, 2^{\Omega}, P\right)$ em que

a) $\Omega$ é um conjunto não vazio finito com $N$ e elementos $w_{1}, w_{2}, \ldots, w_{N}$ denominado espaço amostral do espaço finito de probabilidade;

b) $2^{\Omega}$ é o conjunto das partes de $\Omega$ constituído pelos $2^{N}$ subconjuntos de $\Omega$ denominado espaço dos eventos do espaço finito de probabilidade;

c) $P$ é uma função de probabilidade, isto é, é uma função definida sobre o espaço dos eventos $2^{\Omega}$ cujos valores são números reais não negativos e menores ou iguais a um satisfazendo os três postulados de KOLMOGOROV:

(C1) $\mathrm{P}(\Omega)=1$;

(C2) $\forall A \subset \Omega \longrightarrow 0 \leq P(A) \leq 1$;

(C3) $\forall A, B \subset \Omega$ e $\mathrm{A} \cap \mathrm{B}=\phi \longrightarrow \mathrm{P}(\mathrm{A} \cup \mathrm{B})=\mathrm{P}(\mathrm{A})+\mathrm{P}(\mathrm{B})$.

A fim de construir uma função de probabilidade sobre um conjunto (espaço amostral) finito $\Omega=\left\{w_{1}, w_{2}, \ldots, w_{N}\right\}$ basta definir $P$ sobre os subconjuntos unitários $\left\{w_{j}\right\}$ para $j=1,2, \ldots, N$, isto é, basta escolher números reais não negativos e menores ou iguais a um $p_{1}, p_{2}, \ldots, p_{N}$ de tal modo que $p_{1}+p_{2}+\ldots+p_{N}=1$ e $P\left(\left\{w_{j}\right\}\right)=p_{j}$ para $j=1,2, \ldots, N$.

Assim, para cada $A \subset \Omega, P$ é definido por

$$
P(A)=\sum_{w \in A} P(\{w\})
$$

e $P$ definida desta maneira é uma função que satisfaz os postulados de Kolmogorov.

Dado um conjunto finito $\Omega$, existem infinitos espaços finito de probabilidade $\left(\Omega, 2^{\Omega}, P\right)$ cujo espaço amostral é o conjunto dado $\Omega$ e $P$ é definida da maneira acima. 
Em espaço finito de probabilidade $\left(\Omega, 2^{\Omega}, P\right)$ em que o espaço amostral $\Omega$ tem $N \geq 1$ elementos, existem no máximo $2^{N}-2$ funções distintas de probabilidade associadas a $P$ : para cada subconjunto não vazio $B \subset \Omega \mathrm{e}$ $B \neq \Omega$, com $P(B)>0$, a função $P_{B}$ definida sobre o espaço dos eventos $2^{\Omega}$ por

$$
P_{B}(A)=\frac{P(A \cap B)}{P(B)}, \forall A \subset \Omega
$$

é uma função de probabilidade sobre o espaço amostral $\Omega\left(P_{\Omega}\right.$ coincide com a função de probabilidade $P$ ). Além disso, para cada evento $B \subset \Omega$ com $P(B)>0$, a terna ordenada $\left(\Omega, 2^{\Omega}, P_{B}\right)$ é um espaço finito de probabilidade.

\subsection{ESPAÇO FINITO EQUIPROVÁVEL DE PRO- BABILIDADE}

Um espaço finito equiprovável de probabilidade é uma terna ordenada $\left(\Omega, 2^{\Omega}, P\right)$ cujo espaço amostral $\Omega$ é constituído por $N$ elementos $w_{1}, w_{2}, \ldots, w_{N}$ e cuja função de probabilidade $P$ é definida nos subconjuntos unitários de $\Omega$ por

$$
P\left(\left\{w_{j}\right\}\right)=\frac{1}{N}, \text { para cada } j=1,2, \ldots, N
$$

o que implica que, para cada $A \subset \Omega$,

$$
P(A)=\frac{|A|}{|\Omega|}
$$

em que $|A|$ é o número de elementos do subconjunto de $A$ de $\Omega$.

A definição de um espaço finito ou infinito de probabilidade é dado a seguir. 


\subsection{ESPAÇO DE PROBABILIDADE}

Um espaço de probabilidade é uma terna $(\Omega, F, P)$ em que

a) $\Omega$ é um conjunto não vazio denominado espaço dos eventos do espaço de probabilidade;

b) O espaço dos eventos $F \subset 2^{\Omega}$ do espaço de probabilidade é uma $\sigma$-algebra de subconjuntos de $\Omega$, isto é,

(b1) $\phi \in F$

(b2) Se $A \in F$ então $A^{c} \in F$

(b3) Se $\left(A_{n}\right)_{n \in \mathbb{N}}$ é uma sequência infinita com a propriedade $A_{n} \in F$ para cada $n \in \mathbb{N}$ então

$$
\bigcup_{1}^{\infty} A_{n} \in F
$$

c) $P$ é uma função definida sobre o espaço dos eventos $F$ a qual associa a cada evento um número real não negativo menor ou igual a um que satisfaz os postulados de Kolmogorov

(c1) $P(\Omega)=1$

(c2) $\forall A \subset F \longrightarrow 0 \leq P(A) \leq 1$;

(c3) $P\left(\bigcup_{1}^{\infty} A_{n}\right)=\sum_{1}^{\infty} P\left(A_{n}\right)$ desde que para cada $n \in \mathbb{N}, A_{n} \in F$ e para cada $i, j \in \mathbb{N}=\{1,2,3, \ldots\}, i<j$ e $A_{i} \cap A_{j}=\phi$.

\subsection{NASCIMENTO DE FILHOS}

O nascimento de uma criança constitui um experimento aleatório devido ao genero do recém nascido. O modelo probabilístico é o espaço de probabilidade finito $\left(\Omega, 2^{\Omega}, P\right)$ cujo espaço amostral $\Omega=\{0,1\}$ em que 0 indica nascimento de um filho do sexo feminino e 1 indica o nascimento de um filho do sexo masculino e a função de probabilidade $P$ é calculada de maneira que $P(\{1\})=p$ e $P(\{0\})=1-p$ em que $p$ é um número real não negativo e menor ou igual a um.

No conjunto das famílias com um número fixo $n \geq 2$ de filhos, conhecida a probabilidade $p$, com $0 \leq p \leq 1$, de ocorrência do sexo masculino no nascimento de um filho, e com a hipótese de que o sexo de uma criança é independente dos sexos das outras crianças da família, então a probabilidade de ocorrência, em uma família de $n$ filhos, com 
a) todos os filhos do mesmo sexo é igual a

$$
p^{n}+(1-p)^{n}
$$

b) as primeiras $k \leq n$ crianças mais velhas da família são do sexo masculino e as $n-k$ crianças mais novas do sexo feminino é igual a

$$
p^{k}(1-p)^{n-k}
$$

c) as primeiras $k \leq n$ crianças mais velhas da família são do mesmo sexo, enquanto as demais crianças mais novas $n-k$ são do sexo oposto é igual a

$$
p^{k}(1-p)^{n-k}+(1-p)^{k}(p)^{n-k}
$$

d) existirem exatas $k$ crianças do sexo masculino, com $k \leq n$ e as demais $n-k$ crianças do sexo feminino é igual a

$$
\left(\begin{array}{l}
n \\
k
\end{array}\right) p^{k} \cdot(1-p)^{n-k}
$$

e) existirem pelo menos $k$ crianças do sexo masculino, com $k \leq n$, é igual a

$$
\sum_{j=k}^{n}\left(\begin{array}{l}
n \\
j
\end{array}\right) p^{j} \cdot(1-p)^{n-j}
$$

f) as $s$ crianças mais velhas são meninos e existem um total de $k$ meninos, com $n \geq k \geq s$, é dada por

$$
\left(\begin{array}{l}
n-s \\
k-s
\end{array}\right) p^{k} \cdot(1-p)^{n-k}
$$

g) as $s$ crianças mais velhas são meninos e existem pelo menos $k$ meninos, com $n \geq k \geq s$ é dada por

$$
\sum_{j=k-s}^{n-s}\left(\begin{array}{c}
n-s \\
j
\end{array}\right) p^{s+j} \cdot(1-p)^{n-s-j}
$$

Exemplo 1.5.1. Um casal pretende ter seis filhos, sabendo que estudos genéticos preliminares mostraram que a probabilidade de ter um filho do sexo masculino é o dobro da probabilidade de ter um filho do sexo feminino. Como a probabilidade de ocorrência de um filho do sexo masculino é igual ao dobro da probabilidade de ocorrência de um filho do sexo feminino,

$$
P(\{1\})=\frac{2}{3} \text { e } P(\{0\})=\frac{1}{3}
$$


a) todos os filhos do mesmo sexo é igual a

$$
\left(\frac{2}{3}\right)^{6}+\left(\frac{1}{3}\right)^{6}=\frac{65}{729}
$$

b) as primeiras 4 crianças mais velhas da família são sexo masculino e as 2 crianças mais novas do sexo feminino é igual a

$$
\left(\frac{2}{3}\right)^{4} \cdot\left(\frac{1}{3}\right)^{2}=\frac{16}{729}
$$

c) as primeiras 4 crianças mais velhas da família são do mesmo sexo, enquanto as demais crianças mais novas 2 são do sexo oposto é igual a

$$
\left(\frac{2}{3}\right)^{4} \cdot\left(\frac{1}{3}\right)^{2}+\left(\frac{1}{3}\right)^{4} \cdot\left(\frac{2}{3}\right)^{2}=\frac{20}{729}
$$

d) existirem exatos 4 crianças do sexo masculino e as outras duas crianças do sexo feminino é igual a

$$
\left(\begin{array}{l}
6 \\
4
\end{array}\right)\left(\frac{2}{3}\right)^{4} \cdot\left(\frac{1}{3}\right)^{2}=\frac{240}{729}
$$

e) existir de pelo menos 4 crianças do sexo masculino é igual a

$$
\begin{gathered}
\sum_{j=4}^{6}\left(\begin{array}{l}
6 \\
j
\end{array}\right) \cdot\left(\frac{2}{3}\right)^{j} \cdot\left(\frac{1}{3}\right)^{6-j} \\
=\left(\begin{array}{l}
6 \\
4
\end{array}\right)\left(\frac{2}{3}\right)^{4} \cdot\left(\frac{1}{3}\right)^{2}+\left(\begin{array}{l}
6 \\
5
\end{array}\right)\left(\frac{2}{3}\right)^{5} \cdot\left(\frac{1}{3}\right)+\left(\begin{array}{l}
6 \\
6
\end{array}\right)\left(\frac{2}{3}\right)^{6}=\frac{496}{729}
\end{gathered}
$$

pois o casal poderá ter 4,5 ou 6 filhos do sexo masculino.

f) as 3 crianças mais velhas são meninos e existem um total de 5 meninos é igual

$$
\begin{aligned}
& \left(\begin{array}{l}
6-3 \\
5-3
\end{array}\right)\left(\frac{2}{3}\right)^{5} \cdot\left(\frac{1}{3}\right)^{6-5} \\
& \left(\begin{array}{l}
3 \\
2
\end{array}\right)\left(\frac{2}{3}\right)^{5} \cdot\left(\frac{1}{3}\right)=\frac{96}{729}
\end{aligned}
$$


g) as 3 crianças mais velhas são meninos e existem pelo menos 5 meninos é igual a

$$
\begin{gathered}
\sum_{j=5-3}^{6-3}\left(\begin{array}{c}
6-3 \\
j
\end{array}\right)\left(\frac{2}{3}\right)^{3+j} \cdot\left(\frac{1}{3}\right)^{6-3-j}= \\
=\sum_{j=2}^{3}\left(\begin{array}{l}
3 \\
j
\end{array}\right)\left(\frac{2}{3}\right)^{3+j} \cdot\left(\frac{1}{3}\right)^{3-j} \\
=\left(\begin{array}{l}
3 \\
2
\end{array}\right)\left(\frac{2}{3}\right)^{5} \cdot\left(\frac{1}{3}\right)^{1}+\left(\begin{array}{l}
3 \\
3
\end{array}\right)\left(\frac{2}{3}\right)^{6}=\frac{160}{729} .
\end{gathered}
$$

\subsection{LANÇAMENTO DE MOEDAS}

O lançamento de uma moeda é um experimento aleatório que tem como modelo probabilístico $\left(\Omega, 2^{\Omega}, P\right)$, cujo espaço amostral $\Omega=\{0,1\}$ em que 0 indica a ocorrência de cara e 1 indica a ocorrência de coroa e a função de probabilidade $P$ é calculada de maneira que $P(\{0\})=p$ e $P(\{1\})=1-p$ em que $p$ é um número real não negativo e menor ou igual a um, no caso de moeda viciada e $p=\frac{1}{2}$, no caso de moeda não viciada.

No lançamento de uma moeda $n$ vezes o modelo probabilístico $\left(\Omega, 2^{\Omega}, P\right)$ tem como espaço amostral $\Omega=\{0,1\} \times\{0,1\} \times\{0,1\} \times \ldots \times\{0,1\}$ e a função de probabilidade $P$ calculada no subconjunto unitário $\left\{x_{1}, x_{2}, \ldots, x_{n}\right\}$ em que o número de elementos $x_{j}=0$ iguala $k$, é igual a

$$
P\left(\left\{\left(x_{1}, x_{2}, \ldots, x_{n}\right)\right\}\right)=p^{k}(1-p)^{n-k}
$$

Exemplo 1.6.1. Uma moeda, em que a chance de ocorrência de cara é igual a chance de ocorrência de coroa, é lançada três vezes.

a) a probabilidade de ocorrência de 3 coroas é dada por

$$
\left(\frac{1}{2}\right)^{3}=\frac{1}{8}
$$

b) a probabilidade de ocorrência de exatamente uma coroa é

$$
\left(\begin{array}{l}
3 \\
1
\end{array}\right)\left(\frac{1}{2}\right)^{1}\left(\frac{1}{2}\right)^{2}=\frac{3}{8}
$$


c) a probabilidade de ocorrência de pelo menos uma cara é

$$
\sum_{j=1}^{3}\left(\begin{array}{l}
3 \\
j
\end{array}\right)\left(\frac{1}{2}\right)^{3}=\left[\left(\begin{array}{l}
3 \\
1
\end{array}\right)+\left(\begin{array}{l}
3 \\
2
\end{array}\right)+\left(\begin{array}{l}
3 \\
3
\end{array}\right)\right] \cdot \frac{1}{8}=\frac{7}{8}
$$

A probabilidade de ocorrência de pelo menos uma cara também pode ser calculada por $1-\frac{1}{8}=\frac{7}{8}$, em que $\frac{1}{8}$ é a probabilidade de ocorrência de três coroas.

d) a probabilidade de ocorrência de nenhuma coroa é igual a probabilidade de ocorrência de três caras, ou seja, $\frac{1}{8}$.

e) a probabilidade de ocorrência de no máximo duas caras é dada por:

$$
\sum_{j=1}^{2}\left(\begin{array}{l}
3 \\
j
\end{array}\right)\left(\frac{1}{2}\right)^{3}=\left[\left(\begin{array}{l}
3 \\
1
\end{array}\right)+\left(\begin{array}{l}
3 \\
2
\end{array}\right)\right] \cdot \frac{1}{8}=\frac{6}{8}=\frac{3}{4}
$$

Exemplo 1.6.2. Uma moeda honesta é lançada 6 vezes. A probabilidade de a) ocorrência de ao menos uma cara é igual a

$$
1-\left(\frac{1}{2}\right)^{6}=\frac{63}{64}
$$

b) ocorrência de pelo menos 4 caras é igual a

$$
\sum_{j=4}^{6}\left(\begin{array}{l}
6 \\
j
\end{array}\right)\left(\frac{1}{2}\right)^{6}=\left[\left(\begin{array}{l}
6 \\
4
\end{array}\right)+\left(\begin{array}{l}
6 \\
5
\end{array}\right)+\left(\begin{array}{l}
6 \\
6
\end{array}\right)\right] \cdot \frac{1}{64}=\frac{22}{64}=\frac{11}{32}
$$

Exemplo 1.6.3. Uma moeda honesta é lançada dez vezes. A probabilidade de

a) ocorrência de 5 caras e 5 coroas é igual a

$$
\left(\begin{array}{c}
10 \\
5
\end{array}\right)\left(\frac{1}{2}\right)^{5} \cdot\left(\frac{1}{2}\right)^{5}=\frac{252}{1024}=\frac{63}{256} .
$$

b) ocorrência de 10 caras é igual a

$$
\left(\frac{1}{2}\right)^{10}=\frac{1}{1024}
$$


c) ocorrência de 4 caras e 6 coroas é igual a

$$
\left(\begin{array}{c}
10 \\
4
\end{array}\right)\left(\frac{1}{2}\right)^{4} \cdot\left(\frac{1}{2}\right)^{6}=\frac{210}{1024}=\frac{105}{512} \text {. }
$$

d) ocorrência de pelo menos 8 caras é igual a

$$
\begin{gathered}
\sum_{j=8}^{10}\left(\begin{array}{c}
10 \\
j
\end{array}\right)\left(\frac{1}{2}\right)^{j} \cdot\left(\frac{1}{2}\right)^{10-j} \\
=\sum_{j=8}^{10}\left(\begin{array}{c}
10 \\
j
\end{array}\right)\left(\frac{1}{2}\right)^{10}=\left[\left(\begin{array}{c}
10 \\
8
\end{array}\right)+\left(\begin{array}{c}
10 \\
9
\end{array}\right)+\left(\begin{array}{c}
10 \\
10
\end{array}\right)\right] \cdot \frac{1}{1024}=\frac{7}{128}
\end{gathered}
$$

e) ocorrência de no máximo 3 caras é igual a

$$
\begin{gathered}
\sum_{j=0}^{3}\left(\begin{array}{c}
10 \\
j
\end{array}\right)\left(\frac{1}{2}\right)^{j} \cdot\left(\frac{1}{2}\right)^{10-j}= \\
=\sum_{j=0}^{3}\left(\begin{array}{c}
10 \\
j
\end{array}\right)\left(\frac{1}{2}\right)^{10} \\
=\left[\left(\begin{array}{c}
10 \\
0
\end{array}\right)+\left(\begin{array}{c}
10 \\
1
\end{array}\right)+\left(\begin{array}{c}
10 \\
2
\end{array}\right)+\left(\begin{array}{c}
10 \\
3
\end{array}\right)\right] \cdot \frac{1}{1024}=\frac{176}{1024}=\frac{11}{64}
\end{gathered}
$$

Exemplo 1.6.4. A, B e C se alternam, nessa ordem, jogando uma moeda. O primeiro a tirar cara vence. Qual é a probabilidade de A vencer?

O jogador A pode vencer o jogo na primeira rodada, com probabilidade igual a $\frac{1}{2}$. Caso não vença na primeira rodada, os outros participantes, B e C, não poderão tirar cara, sendo assim o jogador A poderá vencer na segunda rodada com probabilidade $\left(\frac{1}{2}\right)^{3} \cdot \frac{1}{2}=\frac{1}{16}$.

No caso em que A não tirou cara na segunda rodada e B e C também não tiraram cara na segunda rodada, o jogador A poderá vencer na terceira rodada, com probabilidade $\left(\frac{1}{2}\right)^{3} \cdot\left(\frac{1}{2}\right)^{3} \cdot \frac{1}{2}=\frac{1}{128}$ e assim sucessivamente.

As probabilidades do jogador A vencer na primeira rodada ou na segunda rodada ou na terceira rodada e assim sucessivamente, são termos de uma progressão geométrica (P.G.) infinita de primeiro termo $a_{1}=\frac{1}{2}$ e razão 
$q=\frac{1}{8}$. Logo a probabilidade do jogador A vencer será a soma infinita da P.G.,

$$
\frac{1}{2}+\frac{1}{16}+\frac{1}{128}+\ldots=\frac{\frac{1}{2}}{1-\frac{1}{8}}=\frac{4}{7}
$$

\subsection{PROBLEMAS DE PROBABILIDADE COM DA- DOS}

\subsubsection{LANÇAMENTO DE UM DADO}

O experimento aleatório do lançamento de um dado não viciado, em que cada valor do dado tem a mesma chance de ser obtido, tem como modelo probabilístico o espaço finito equiprovável de probabilidade $\left(\Omega, 2^{\Omega}, P\right)$, cujo espaço amostral $\Omega$ é dado por

$$
\Omega=\{1,2,3,4,5,6\}
$$

A probabilidade de ocorrência do valor 1 ou do valor 6 é a probabilidade $P(A)$ do evento $A=\{1,6\} \subset \Omega$ calculado por

$$
P(A)=\frac{|A|}{|\Omega|}=\frac{2}{6}=\frac{1}{3}
$$

A probabilidade de ocorrência de um número primo é a probabilidade $P(B)$ do evento $B=\{2,3,5\} \subset \Omega$ calculado por

$$
P(B)=\frac{|B|}{|\Omega|}=\frac{3}{6}=\frac{1}{2}
$$

Exemplo 1.7.1. Um dado é viciado, de modo que a probabilidade de observarmos um número na face de cima é proporcional a esse número. Como o espaço amostral é não equiprovável, vamos inicialmente determinar a probabilidade de cada evento elementar. Seja $P(i)$ a probabilidade de no lançamento de um dado ocorrer o resultado $i \in \Omega=\{1,2,3,4,5,6\}$. Temos:

- $P(2)=2 P(1)$

- $P(3)=3 P(1)$

- $P(4)=4 P(1)$ 
- $P(5)=5 P(1)$

- $P(6)=6 P(1)$

$$
\begin{gathered}
\sum_{i=1}^{6} P(i)=1 \Longrightarrow P(1)+2 P(1)+2 P(1)+3 P(1)+4 P(1)+5 P(1)+6 P(1)=1 \\
\Longrightarrow P(1)=\frac{1}{21}
\end{gathered}
$$

Então:

$$
\begin{aligned}
& P(1)=\frac{1}{21}, P(2)=\frac{2}{21}, P(3)=\frac{3}{21}, P(4)=\frac{4}{21}, P(5)=\frac{5}{21} \text { e } P(6)= \\
& \frac{6}{21} \text {. }
\end{aligned}
$$

a) a probabilidade de ocorrer número par é igual a

$$
P\{2,4,6\}=\frac{2}{21}+\frac{4}{21}+\frac{6}{21}=\frac{12}{21}=\frac{4}{7} .
$$

b) a probabilidade de ocorrer número maior ou igual a 5 .

$$
P\{5,6\}=\frac{5}{21}+\frac{6}{21}=\frac{11}{21} .
$$

\subsubsection{LANÇAMENTO SIMULTÂNEO DE DOIS DADOS DISTINTOS}

O experimento aleatório do lançamento de dois dados não viciados de cores diferentes (um branco e um vermelho) em que a chance de um valor qualquer em cada um dos dados é a mesma e igual para todos os valores tem como modelo probabilístico o espaço finito equiprovável de probablidade $\left(\Omega, 2^{\Omega}, P\right)$ cujo espaço amostral $\Omega$ é

$$
\Omega=\{(i, j): i, j \in\{1,2,3,4,5,6\}\}
$$

\section{Exemplo 1.7.2.}

a) A probabilidade de ocorrência de soma 7 ou 8 pontos nos dois dados é a probabilidade do evento $A \cup B$ em que

$$
A=\{(1,6),(2,5),(3,5),(4,3),(5,2),(6,1)\}
$$




$$
B=\{(2,6),(3,5),(4,4),(5,3),(6,2)\}
$$

calculada por

$$
P(A \cup B)=P(A)+P(B)=\frac{|A \cup B|}{|\Omega|}=\frac{|A|}{|\Omega|}+\frac{|B|}{|\Omega|}=\frac{6}{36}+\frac{5}{36}=\frac{11}{36}
$$

b) A probabilidade de ocorrerem números iguais é dada pela probabilidade do evento $A=\{(1,1),(2,2),(3,3),(4,4),(5,5),(6,6)\}$, ou seja

$$
P(A)=\frac{|A|}{|\Omega|}=\frac{6}{36}=\frac{1}{6}
$$

c) A probabilidade de ocorrerem números diferentes é a probabilidade do evento $\bar{A}$, dada por:

$$
P(\bar{A})=1-P(A)=1-\frac{1}{6}=\frac{5}{6}
$$

d) A probabilidade da soma dos números ser 12 é a probabilidade do evento $C=\{(6,6)\}$ dada por

$$
P(C)=\frac{|C|}{|\Omega|}=\frac{1}{36}
$$

e) A probabilidade de aparecer número 3 em ao menos um dado é a probabilidade do evento $D=\{(1,3),(2,3),(3,1),(3,2),(3,3),(3,4),(3,5),(3,6),(4,3),(5,3),(6,3)\}$, calculada por

$$
P(D)=\frac{|D|}{|\Omega|}=\frac{11}{36}
$$

f) a probabilidade da soma dos pontos ser 6 , se a face observada de $d_{1}$ for 2 é a probabilidade do evento $E \cup F$ em que $E=\{(1,5),(2,4),(3,3),(4,2),(5,1)\}$ e $F=\{(2,1),(2,2),(2,3),(2,4),(2,5),(2,6)\}$ calculada por

$$
\begin{gathered}
P(E \cup F)=P(E)+P(F)-P(E \cap F) \\
=\frac{|E|}{|\Omega|}+\frac{|F|}{|\Omega|}-\frac{|E \cap F|}{|\Omega|}=\frac{5}{36}+\frac{6}{36}-\frac{1}{36}=\frac{5}{18}
\end{gathered}
$$

g) a probabilidade de o segundo dado sair com o valor maior do que o primeiro é a probabilidade do evento

$$
\begin{gathered}
G=\{(2,1),(3,1),(3,2),(4,1),(4,2),(4,3),(5,1),(5,2), \\
(5,3),(5,4),(6,1),(6,2),(6,3),(6,4),(6,5)\}
\end{gathered}
$$

que é igual a

$$
P(G)=\frac{|G|}{|\Omega|}=\frac{15}{36}=\frac{5}{12}
$$


Exemplo 1.7.3. Dois dados distintos são lançados n vezes em sequência. Determine quão grande deve ser o valor de $\mathrm{n}$ para que a probabilidade de que um duplo 6 apareça pelo menos uma vez seja pelo menos igual a $1 / 2$.

Em um único lançamento de dois dados distintos (um verde e um amarelo), a probabilidade de que ocorra um duplo 6 é $\frac{1}{36}$. Já em dois lançamentos desses dois dados a probabilidade de que ocorra pelo menos um duplo 6 é dada por

$$
1-\left(\frac{35}{36}\right)^{2}
$$

e em $n$ lançamentos, a probabilidade de que ocorra pelo menos um duplo 6 é dada por

$$
1-\left(\frac{35}{36}\right)^{n}
$$

O valor de $n$ para que a probabilidade de que um duplo 6 apareça pelo menos uma vez seja pelo menos igual a $1 / 2$, ou seja

$$
\begin{gathered}
1-\left(\frac{35}{36}\right)^{n} \geq \frac{1}{2} \\
\Longrightarrow\left(\frac{35}{36}\right)^{n} \leq \frac{1}{2} \\
\Longrightarrow n \cdot[\log 35-\log 36] \leq \log 1-\log 2 \\
\Longrightarrow-0,02 \cdot n \leq-0,30 \\
\Longrightarrow n \geq 15
\end{gathered}
$$

$\operatorname{logo}$, para $n \geq 15$ teremos a probabilidade de que um duplo 6 apareça pelo menos uma vez seja pelo menos igual a $\frac{1}{2}$.

\subsubsection{LANÇAMENTO SIMULTÂNEO DE CINCO DADOS DISTINTOS}

No lançamento simultâneo de 5 dados distintos (um amarelo, um branco, um verde, um vermelho e um preto) ou cinco lançamentos sucessivos de um mesmo dado, a probabilidade de que a soma dos cinco valores obtidos é igual a 20 é igual ao coeficiente de $t^{20}$ no produto

$$
\left(t+t^{2}+t^{3}+t^{4}+t^{5}+t^{6}\right)^{5}
$$




$$
=t^{5} \cdot \frac{\left(1-t^{6}\right)^{5}}{(1-t)^{5}}
$$

Como

$$
\left(1-t^{6}\right)^{5}=-t^{30}+5 t^{24}-10 t^{18}+10 t^{12}-5 t^{6}+1
$$

os coeficientes em $\left(1-t^{6}\right)^{5}(1-t)^{-5}$ dos termos em $t^{15}, t^{9}$ e $t^{3}$ são respectivamente iguais a

$$
\begin{gathered}
\left(\begin{array}{c}
-5 \\
15
\end{array}\right) \cdot(-1)^{15}=\left(\begin{array}{c}
5+15-1 \\
15
\end{array}\right)=\left(\begin{array}{l}
19 \\
15
\end{array}\right)=3876 \\
\left(\begin{array}{c}
-5 \\
9
\end{array}\right) \cdot(-1)^{9}=\left(\begin{array}{c}
5+9-1 \\
9
\end{array}\right)=\left(\begin{array}{c}
13 \\
9
\end{array}\right)=715 \\
\left(\begin{array}{c}
-5 \\
3
\end{array}\right) \cdot(-1)^{3}=\left(\begin{array}{c}
5+3-1 \\
9
\end{array}\right)=\left(\begin{array}{l}
7 \\
3
\end{array}\right)=35
\end{gathered}
$$

O coeficiente de $t^{15}$ do desenvolvimento de $\left(1-t^{6}\right)^{5} \cdot(1-t)^{-5}$ é

$$
1 \cdot 3876-5 \cdot 715+10 \cdot 35=651
$$

A probabilidade de ocorrência de soma igual a 20 no lançamento de 5 dados distintos é dada por

$$
\frac{651}{6^{5}}=\frac{651}{7776}=\frac{217}{2592}
$$

Observação: Para $n$ inteiro positivo e $p$ inteiro não negativo, por definição

$$
\left(\begin{array}{c}
-n \\
p
\end{array}\right)(-1)^{p}=\left(\begin{array}{c}
n+p-1 \\
p
\end{array}\right)
$$

de fato,

$$
\begin{gathered}
\left(\begin{array}{c}
-n \\
p
\end{array}\right)(-1)^{p}=\frac{(-n)(-n-1) \ldots(-n-p+1)(-1)^{p}}{p !} \\
=\frac{(-1)^{p}(n)(n+1) \ldots(n+p-1)(-1)^{p}}{p !} \\
=\frac{(n)(n+1) \ldots(n+p-1)}{p !} \\
=\frac{(n+p-1) !}{p !(n-1) !} \\
=\left(\begin{array}{c}
n+p-1 \\
p
\end{array}\right) .
\end{gathered}
$$




\subsection{BARALHO}

O baralho padrão é constituído por 52 cartas com quatro naipes diferentes: $\diamond$ (ouro), $\diamond$ (copas), $\boldsymbol{\phi}$ (espadas) e $\boldsymbol{\beta}$ (paus) e os valores das cartas são A (ás), 2, 3, 4, 5, 6, 7, 8, 9, 10, J (valete), Q (dama) e K (rei)

O experimento aleatório da retirada simultânea de duas cartas em que todas as cartas tem chances iguais de serem retiradas tem como modelo probabilístico $\left(\Omega, 2^{\Omega}, P\right)$, cujo espaço amostral $\Omega$ é o conjunto constituído de todos os $\left(\begin{array}{c}52 \\ 2\end{array}\right)$ subconjuntos com dois elementos do conjunto $C$ das cartas do baralho.

A probabilidade de obtenção de dois ases é a probabilidade do evento $A$ que é o conjunto de todos os $\left(\begin{array}{l}4 \\ 2\end{array}\right)$ subconjuntos de dois elementos do conjunto dos quatro ases do baralho calculada por

$$
P(A)=\frac{|A|}{|\Omega|}=\frac{\left(\begin{array}{l}
4 \\
2
\end{array}\right)}{\left(\begin{array}{c}
52 \\
2
\end{array}\right)}
$$

O experimento aleatório da retirada de duas cartas em sequência uma após a outra sem devolução da primeira carta ao baralho tem como modelo probabilístico o espaço finito equiprovável de probabilidade $\left(\Omega, 2^{\Omega}, P\right)$ cujo espaço amostral $\Omega$ é dado por

$$
\Omega=\{(x, y): x, y \in C \text { e } x \neq y\}
$$

e a probabilidade de obtenção de dois ases é a probabilidade do evento $A$ constituído pelos pares ordenados cujos elementos são elementos distintos do conjunto dos ases do baralho, calculada por

$$
P(A)=\frac{|A|}{|\Omega|}=\frac{4 \cdot 3}{52 \cdot 51}
$$

que é a mesma probabilidade calculada anteriormente para cartas retiradas simultaneamente.

O experimento aleatório de obtenção de duas cartas retiradas em sequência uma após a outra com a reposição da carta retirada na primeira vez, em que a chance de retirada de uma carta é a mesma e igual para todas as cartas, tem como modelo probabilístico o espaço finito equiprovável de probabilidade $\left(\Omega, 2^{\Omega}, P\right)$ cujo espaço amostral $\Omega$ é dado por

$$
\Omega=\{(x, y): x, y \in C\}=C \times C
$$

A probabilidade de ocorrência de duas cartas de ouros é a probabilidade do evento $C_{\diamond} \mathrm{X} C_{\diamond}$ em que $C_{\diamond}$ é o conjunto das treze cartas de ouro 
calculada por

$$
P\left(C_{\diamond} \mathrm{X} C_{\diamond}\right)=\frac{\left|C_{\diamond} \mathrm{X} C_{\diamond}\right|}{|C \mathrm{X} C|}=\frac{13 \cdot 13}{52 \cdot 52}
$$

e a probabilidade de ocorrência de duas cartas do mesmo naipe é a probabilidade do evento $C_{\diamond} \mathrm{X} C_{\diamond} \cup C_{\odot} \mathrm{X} C_{\odot} \cup C_{\boldsymbol{\wedge}} \mathrm{X} C_{\boldsymbol{\phi}} \cup C_{\boldsymbol{\Delta}} \mathrm{X} C_{\boldsymbol{\beta}}$ cujo valor é igual a $4 \cdot \frac{13 \cdot 13}{52 \cdot 52}$.

Exemplo 1.8.1. Oito cartas são retiradas aleatoriamente de um baralho padrão com 52 cartas em que a chance de escolha de uma carta é a mesma para todas as cartas do baralho. A probabilidade de ocorrência de exatamente 4 das 8 cartas são pretas (naipes espadas e paus) e 4 das 8 cartas são vermelhas (naipes copas e ouros) é igual a

$$
\frac{\left(\begin{array}{c}
26 \\
4
\end{array}\right)\left(\begin{array}{c}
26 \\
4
\end{array}\right)}{\left(\begin{array}{c}
52 \\
8
\end{array}\right)}
$$

Quando uma quantidade par qualquer de cartas é retirada aleatoriamente de um baralho, a probabilidade de ocorrência de metade das cartar ser vermelhas e metade das cartas ser preta passa a ser calculada por

$$
\frac{\left(\begin{array}{c}
26 \\
1
\end{array}\right)\left(\begin{array}{c}
26 \\
1
\end{array}\right)+\left(\begin{array}{c}
26 \\
2
\end{array}\right)\left(\begin{array}{c}
26 \\
2
\end{array}\right)+\ldots+\left(\begin{array}{c}
26 \\
26
\end{array}\right)\left(\begin{array}{c}
26 \\
26
\end{array}\right)}{\left(\begin{array}{c}
52 \\
2
\end{array}\right)+\left(\begin{array}{c}
52 \\
4
\end{array}\right)+\ldots+\left(\begin{array}{c}
52 \\
52
\end{array}\right)}
$$

que é igual a

$$
\frac{\frac{52 !}{26 ! \cdot 26 !}-1}{2^{51}-1}
$$

lembrando que

$$
\begin{aligned}
&\left(\begin{array}{c}
52 \\
0
\end{array}\right)+\left(\begin{array}{c}
52 \\
2
\end{array}\right)+\ldots+\left(\begin{array}{c}
52 \\
52
\end{array}\right)=\left(\begin{array}{c}
52 \\
1
\end{array}\right)+\left(\begin{array}{c}
52 \\
3
\end{array}\right)+\ldots+\left(\begin{array}{c}
52 \\
51
\end{array}\right) \\
&=\frac{1}{2}\left[\sum_{j=0}^{52}\left(\begin{array}{c}
52 \\
j
\end{array}\right)-\sum_{j=0}^{52}(-1)^{j}\left(\begin{array}{c}
52 \\
j
\end{array}\right)\right] \\
&=2^{51}
\end{aligned}
$$

e que

$$
\left(\begin{array}{c}
26 \\
0
\end{array}\right)^{2}+\left(\begin{array}{c}
26 \\
1
\end{array}\right)^{2}+\ldots+\left(\begin{array}{c}
26 \\
26
\end{array}\right)^{2}=\left(\begin{array}{c}
52 \\
26
\end{array}\right)=\frac{52 !}{26 ! \cdot 26 !}
$$


O modelo probabilístico é o espaço de probabilidade finito não equiprovável $\left(\Omega, 2^{\Omega}, P\right)$ cujo espaço amostral $\Omega$ é constituído pela totalidade dos conjuntos com 2 elementos, dos subconjuntos com 4 elementos e assim por diante até o subconjunto com os 52 elementos do conjunto das 52 cartas.

\subsubsection{PÔQUER}

Considerando $C$ o conjunto das 52 cartas de um baralho como a união dijunta

$$
C=C_{1} \cup C_{2} \cup C_{3} \cup C_{4} \cup C_{5} \cup C_{6} \cup C_{7} \cup C_{8} \cup C_{9} \cup C_{10} \cup C_{11} \cup C_{12} \cup C_{13}
$$

em que $C_{1}$ é o subconjunto dos 4 ases, $C_{8}$ é o subconjunto dos 4 oitos, $C_{11}$ é o subconjunto dos 4 valetes, $C_{12}$ é o subconjunto das 4 damas e $C_{13}$ é o subconjunto dos 4 reis. Mãos de pôquer são subconjuntos de cinco cartas de um baralho comum e são consideradas como amostras não ordenadas de cinco cartas de um baralho de 52 cartas.

Com a hipótese de que todas as cartas tem a mesma chance de constituir uma mão de pôquer, a probabilidade de ocorrência de uma mão de pôquer com exatos

a) UM ÁS é $\frac{\left(\begin{array}{l}4 \\ 1\end{array}\right) \cdot\left(\begin{array}{c}48 \\ 4\end{array}\right)}{\left(\begin{array}{c}52 \\ 5\end{array}\right)}$

b) DOIS ASES é $\frac{\left(\begin{array}{l}4 \\ 2\end{array}\right) \cdot\left(\begin{array}{c}48 \\ 3\end{array}\right)}{\left(\begin{array}{c}52 \\ 5\end{array}\right)}$

c) TRÊS ASES é $\frac{\left(\begin{array}{l}4 \\ 3\end{array}\right) \cdot\left(\begin{array}{c}48 \\ 2\end{array}\right)}{\left(\begin{array}{c}52 \\ 5\end{array}\right)}$

d) QUATRO ASES é $\frac{\left(\begin{array}{l}4 \\ 4\end{array}\right) \cdot\left(\begin{array}{c}48 \\ 1\end{array}\right)}{\left(\begin{array}{c}52 \\ 5\end{array}\right)}$

e) UM PAR

Para simplificar, considere uma mão com dois ases, um dois, um sete e uma dama.

A escolha de dois ases entre quatro ases possíveis é dada por $\left(\begin{array}{l}4 \\ 2\end{array}\right)=6$ combinações com os quatro ases. A escolha de um dois é dada por $\left(\begin{array}{l}4 \\ 1\end{array}\right)=4$ combinações, assim como a escolha de um sete e de uma dama. Cada par pode ser combinado com as outras três cartas de $\left(\begin{array}{l}4 \\ 2\end{array}\right)\left(\begin{array}{l}4 \\ 1\end{array}\right)\left(\begin{array}{l}4 \\ 1\end{array}\right)\left(\begin{array}{l}4 \\ 1\end{array}\right)=384$ maneiras. 
Para determinar o número de mãos de pôquer com um único par, a escolha do par é feita de $\left(\begin{array}{c}13 \\ 1\end{array}\right)$ possibilidades e a escolha das outras três cartas é igual a $\left(\begin{array}{c}12 \\ 3\end{array}\right)$. Logo, a probabilidade de ocorrer um par em uma mão de pôquer é calculada por:

$$
\frac{\left(\begin{array}{c}
13 \\
1
\end{array}\right)\left(\begin{array}{c}
12 \\
3
\end{array}\right)\left(\begin{array}{c}
4 \\
2
\end{array}\right)\left(\begin{array}{l}
4 \\
1
\end{array}\right)\left(\begin{array}{l}
4 \\
1
\end{array}\right)\left(\begin{array}{l}
4 \\
1
\end{array}\right)}{\left(\begin{array}{c}
52 \\
5
\end{array}\right)}
$$

\section{f) DOIS PARES}

Inicialmente, considere uma mão de pôquer com dois ases, dois reis e um sete.

A escolha de dois ases é dado por $\left(\begin{array}{l}4 \\ 2\end{array}\right)=6$ combinações e o mesmo número é dado para a escolha de dois reis. A escolha de um sete entre os 4 do baralho é dada por $\left(\begin{array}{l}4 \\ 1\end{array}\right)=4$ combinações. Cada par de ases, pode ser combinado com cada par de reis e com cada sete de $\left(\begin{array}{l}4 \\ 2\end{array}\right)\left(\begin{array}{l}4 \\ 2\end{array}\right)\left(\begin{array}{l}4 \\ 1\end{array}\right)$ maneiras. Dentre os 13 valores das cartas tem-se $\left(\begin{array}{c}13 \\ 2\end{array}\right)$ combinações de dois pares e $\left(\begin{array}{c}11 \\ 1\end{array}\right)$ combinações para a escolha da quinta carta cujo valor é distinto das cartas dos dois pares.

Logo, a probabilidade de obtenção de dois pares em uma mão de pôquer é calculada por

$$
\frac{\left(\begin{array}{c}
13 \\
2
\end{array}\right)\left(\begin{array}{c}
11 \\
1
\end{array}\right)\left(\begin{array}{c}
4 \\
2
\end{array}\right)\left(\begin{array}{c}
4 \\
2
\end{array}\right)\left(\begin{array}{c}
4 \\
1
\end{array}\right)}{\left(\begin{array}{c}
52 \\
5
\end{array}\right)}
$$

g) THREE OF A KIND (Um trio)

Concidere a ocorrência de uma mão de pôquer com três ases, um sete e uma dama cuja a probabilidade é dado por

$$
\frac{\left(\begin{array}{l}
4 \\
3
\end{array}\right)\left(\begin{array}{l}
4 \\
1
\end{array}\right)\left(\begin{array}{l}
4 \\
1
\end{array}\right)}{\left(\begin{array}{c}
52 \\
5
\end{array}\right)}
$$

que é igual a probabilidade de ocorrência de uma mão de pôquer com três cartas de $C_{i}$, uma carta de $C_{j}$ e uma carta de $C_{k}$ em que $\{i, j, k\}$ é um subconjunto fixado de $\{1,2, \ldots, 13\}$.

A probabilidade de ocorrência de mão de pôquer com três cartas de $C_{i}$, uma carta de $C_{j}$ e uma carta de $C_{k}$ em que $\{i, j, k\}$ é um subconjunto qualquer de $\{1,2, \ldots, 13\}$ é igual a

$$
\frac{\left(\begin{array}{c}
13 \\
1
\end{array}\right)\left(\begin{array}{c}
12 \\
2
\end{array}\right)\left(\begin{array}{l}
4 \\
3
\end{array}\right)\left(\begin{array}{l}
4 \\
1
\end{array}\right)\left(\begin{array}{l}
4 \\
1
\end{array}\right)}{\left(\begin{array}{c}
52 \\
5
\end{array}\right)}
$$


desde de que há $\left(\begin{array}{c}13 \\ 1\end{array}\right)$ possibilidades para $C_{i}$ e $\left(\begin{array}{c}12 \\ 2\end{array}\right)$ possibilidades para $C_{j}$ e $C_{k}$.

h) FULL HOUSE (Um trio e um par)

A probabilidade de ocorrência de uma mão de pôquer com três ases e dois reis é igual a

$$
\frac{\left(\begin{array}{l}
4 \\
3
\end{array}\right)\left(\begin{array}{l}
4 \\
2
\end{array}\right)}{\left(\begin{array}{c}
52 \\
5
\end{array}\right)}
$$

que é igual a probabilidade de ocorrência de uma mão de pôquer com três cartas de $C_{i}$ e duas cartas de $C_{j}$ em que $\{i, j\}$ é um subconjunto fixado de $\{1,2, \ldots, 13\}$.

A probabilidade de ocorrência de mão de pôquer com três cartas de $C_{i}$ e duas cartas de $C_{j}$ em que $\{i, j\}$ é um subconjunto qualquer de $\{1,2, \ldots, 13\}$ é igual a

$$
\frac{\left(\begin{array}{c}
13 \\
1
\end{array}\right)\left(\begin{array}{c}
12 \\
1
\end{array}\right)\left(\begin{array}{l}
4 \\
3
\end{array}\right)\left(\begin{array}{l}
4 \\
2
\end{array}\right)}{\left(\begin{array}{c}
52 \\
5
\end{array}\right)}
$$

desde de que há $\left(\begin{array}{c}13 \\ 1\end{array}\right)$ possibilidades para $C_{i}$ e $\left(\begin{array}{c}12 \\ 2\end{array}\right)$ possibilidades para $C_{j}$

i) FLUSH (Cinco cartas do mesmo naipe)

A probabilidade de ocorrência de uma mão de pôquer com cinco cartas de ouro é igual a

$$
\frac{\left(\begin{array}{c}
13 \\
5
\end{array}\right)}{\left(\begin{array}{c}
52 \\
5
\end{array}\right)}
$$

e a probablidade de ocorrência de uma mão de pôquer com cinco cartas de mesmo naipe é igual a

$$
4 \cdot \frac{\left(\begin{array}{c}
13 \\
5
\end{array}\right)}{\left(\begin{array}{c}
52 \\
5
\end{array}\right)}
$$

\section{j) SEQUÊNCIAS}

A probabilidade de ocorrência de uma mão de pôquer com um três, um quatro, um cinco, um seis e um sete em que as cartas não são necessariamente do mesmo naipe é igual

$$
\frac{\left(\begin{array}{l}
4 \\
1
\end{array}\right)\left(\begin{array}{l}
4 \\
1
\end{array}\right)\left(\begin{array}{l}
4 \\
1
\end{array}\right)\left(\begin{array}{l}
4 \\
1
\end{array}\right)\left(\begin{array}{l}
4 \\
1
\end{array}\right)}{\left(\begin{array}{c}
52 \\
5
\end{array}\right)}
$$


e a probabilidade de ocorrência de uma mão de pôquer com uma carta de $C_{i}$, uma carta de $C_{i+1}$, uma carta de $C_{i+2}$, uma carta de $C_{i+3}$ e uma carta de $C_{i+4}$ em que $\{i\}$ é im subconjunto qualquer de $\{1,2, \ldots, 10\}$ (a sequência 10, J (valete), Q (dama), K (rei), A (ás) é possível em uma mão de pôquer) é igual a

$$
\frac{\left(\begin{array}{c}
10 \\
1
\end{array}\right)\left(\begin{array}{l}
4 \\
1
\end{array}\right)\left(\begin{array}{l}
4 \\
1
\end{array}\right)\left(\begin{array}{l}
4 \\
1
\end{array}\right)\left(\begin{array}{l}
4 \\
1
\end{array}\right)\left(\begin{array}{l}
4 \\
1
\end{array}\right)}{\left(\begin{array}{c}
52 \\
5
\end{array}\right)}
$$

A probabilidade de ocorrência de uma mão de pôquer com sequências de cinco cartas de um mesmo naipe é igaul a

$$
\frac{\left(\begin{array}{c}
10 \\
1
\end{array}\right)\left(\begin{array}{l}
4 \\
1
\end{array}\right)}{\left(\begin{array}{c}
52 \\
5
\end{array}\right)}
$$

\subsubsection{PÔQUER COM DADOS}

Pôquer com dados é jogado com o lançamento simultâneo de 5 dados. O experimento aleatório do lançamento de cinco dados não viciados de cores diferentes em que a chance de um valor qualquer em cada um dos dados é o mesmo e igual para todos os valores tem como modelo probabilístico o espaço finito equiprovável de probablidade $\left(\Omega, 2^{\Omega}, P\right)$ cujo espaço amostral $\Omega$ é

$$
\Omega=\{(i, j, k, l, m): i, j, k, l, m \in\{1,2,3,4,5,6\}\}
$$

A probabilidade de ocorrência de nenhum dado com o mesmo valor é a probabilidade do evento $A$ calculada por

$$
P(A)=\frac{6}{6} \cdot \frac{5}{6} \cdot \frac{4}{6} \cdot \frac{3}{6} \cdot \frac{2}{6}=\frac{120}{1296} \cong 0,0926
$$

A probabilidade de ocorrência de um par é a probabilidade do evento $B$ calculada por

$$
P(B)=\left(\begin{array}{l}
5 \\
2
\end{array}\right) \cdot \frac{6}{6} \cdot \frac{1}{6} \cdot \frac{5}{6} \cdot \frac{4}{6} \cdot \frac{3}{6}=\frac{600}{1296} \cong 0,4630
$$

A probabilidade de ocorrência de dois pares é a probabilidade do evento $C$ calculada por

$$
P(C)=\frac{5 !}{2 ! 2 !} \cdot \frac{6}{6} \cdot \frac{1}{6} \cdot \frac{5}{6} \cdot \frac{1}{6} \cdot \frac{4}{6}=\frac{300}{1296} \cong 0,2315
$$


A probabilidade de ocorrência de uma trinca é a probabilidade do evento $D$ calculada por

$$
P(D)=\left(\begin{array}{l}
5 \\
3
\end{array}\right) \cdot \frac{6}{6} \cdot \frac{1}{6} \cdot \frac{1}{6} \cdot \frac{5}{6} \cdot \frac{4}{6}=\frac{200}{1296} \cong 0,1543
$$

A probabilidade de ocorrência de uma trinca e um par é a probabilidade do evento $E$ calculada por

$$
P(E)=\left(\begin{array}{l}
5 \\
3
\end{array}\right) \cdot \frac{6}{6} \cdot \frac{1}{6} \cdot \frac{1}{6} \cdot \frac{5}{6} \cdot \frac{1}{6}=\frac{50}{1296} \cong 0,0386
$$

A probabilidade de ocorrência de quatro resultados iguais é a probabilidade do evento $F$ calculada por

$$
P(F)=\left(\begin{array}{l}
5 \\
4
\end{array}\right) \cdot \frac{6}{6} \cdot \frac{1}{6} \cdot \frac{1}{6} \cdot \frac{1}{6} \cdot \frac{5}{6}=\frac{25}{1296} \cong 0,0193
$$

A probabilidade de ocorrência de cinco resultados iguais é a probabilidade do evento $G$ calculada por

$$
P(G)=\frac{6}{6} \cdot \frac{1}{6} \cdot \frac{1}{6} \cdot \frac{1}{6} \cdot \frac{1}{6}=\frac{1}{1296} \cong 0,0008
$$

As propriedades apresentadas a seguir em espaços finitos de probabilidade são válidas em espaços de probabilidade quaisquer e as demonstrações das propriedades em espaços de probabilidade quaisquer são praticamente as mesmas provas para espaços finitos de probabilidade.

\subsection{PROPRIEDADES}

Seja $\left(\Omega, 2^{\Omega}, P\right)$ um espaço finito de probabilidade então

a) $\mathbf{P}(\phi)=\mathbf{0}$

Demonstração. Como $\Omega=\Omega \cup \Omega$ e $\Omega \cap \phi=\phi$

$\mathrm{P}(\Omega)=\mathrm{P}(\Omega \cup \phi)$

e pelo terceiro postulado de Kolmogorov,

$\mathrm{P}(\Omega)=\mathrm{P}(\Omega)+\mathrm{P}(\phi)$

o que mostra que

$\mathrm{P}(\phi)=0$. 
b) $\mathbf{P}(\bar{A})=\mathbf{1}-\mathbf{P}(\mathbf{A})$

Demonstração. Como $\Omega=\mathrm{A} \cup \bar{A}$ e $\mathrm{A} \cap \bar{A}=\phi$

$P(\Omega)=P(A \cup \bar{A})$

e pelo terceiro postulado de Kolmogorov

$1=\mathrm{P}(\Omega)=\mathrm{P}(\mathrm{A})+\mathrm{P}(\bar{A})$

o que mostra que

$\mathrm{P}(\bar{A})=1-P(A)$

c) $\mathbf{P}(\mathbf{A} \backslash \mathbf{B})=\mathbf{P}(\mathrm{A})-\mathbf{P}(\mathrm{A} \cap \mathrm{B})$

Demonstração. Como $A \backslash B=A \cap \bar{B}, A=(A \backslash B) \cup(A \cap B)$ e $(A \backslash B) \cap(A \cap B)=\phi$, pelo terceiro postulado de Kolmogorov

$\mathrm{P}(\mathrm{A})=\mathrm{P}((\mathrm{A} \backslash \mathrm{B}) \cup(\mathrm{A} \cap \mathrm{B}))=\mathrm{P}(\mathrm{A} \backslash \mathrm{B})+\mathrm{P}(\mathrm{A} \cap \mathrm{B})$

o que mostra que

$\mathrm{P}(\mathrm{A} \backslash \mathrm{B})=\mathrm{P}(\mathrm{A})-\mathrm{P}(\mathrm{A} \cap \mathrm{B})$

d) Se $\mathbf{A} \subset \mathbf{B}$, então $\mathbf{P}(\mathbf{A}) \leq \mathbf{P}(\mathbf{B})$

Demonstração. Como $\mathrm{A} \subset \mathrm{B}$, então $\mathrm{A} \cap \mathrm{B}=\mathrm{A}, \operatorname{logo} \mathrm{P}(\mathrm{A} \cap \mathrm{B})=$ $\mathrm{P}(\mathrm{A})$ e pela propriedade anterior,

$\mathrm{P}(\mathrm{B} \backslash \mathrm{A})=\mathrm{P}(\mathrm{B})-\mathrm{P}(\mathrm{A} \cap \mathrm{B})$, ou seja,

$\mathrm{P}(\mathrm{B} \backslash \mathrm{A})=\mathrm{P}(\mathrm{B})-\mathrm{P}(\mathrm{A}) \geq 0$ o que mostra que

$\mathrm{P}(\mathrm{B})-\mathrm{P}(\mathrm{A}) \geq 0$ ou $\mathrm{P}(\mathrm{A}) \leq \mathrm{P}(\mathrm{B})$

Em particular, como $A \subset \Omega, P(A) \leq P(\Omega)=1$.

e) $\forall \mathbf{A}, \mathbf{B} \subset \Omega \Longrightarrow \mathbf{P}(\mathbf{A} \cup \mathbf{B}) \cdot \mathbf{P}(\mathbf{A} \cap \mathbf{B}) \leq \mathbf{P}(\mathbf{A}) \cdot \mathbf{P}(\mathbf{B})$ 
Demonstração. Pelo terceiro postulado de Kolmogorov,

$$
\begin{gathered}
P(A \cup B)=P(A \cap B)+P(A \cap \bar{B})+P(\bar{A} \cap B) \\
P(A)=P(A \cap B)+P(A \cap \bar{B}) \\
P(B)=P(A \cap B)+P(\bar{A} \cap B)
\end{gathered}
$$

Multiplicando (2) e (3), temos:

$$
\begin{aligned}
P(A) \cdot P(B)= & P(A \cap B) \cdot P(A \cap B)+P(A \cap B) \cdot P(\bar{A} \cap B) \\
+ & P(A \cap B) \cdot P(A \cap \bar{B})+P(\bar{A} \cap B) \cdot P(A \cap \bar{B}) \\
\geq & P(A \cap B) \cdot P(A \cap B)+P(A \cap B) \cdot P(\bar{A} \cap B)+P(A \cap B) \cdot P(A \cap \bar{B}) \\
\geq & P(A \cap B) \cdot[P(A \cap B)+P(\bar{A} \cap B)+P(A \cap \bar{B})], \text { de }(1), \text { temos: } \\
& P(A) \cdot P(B) \geq P(A \cup B) \cdot P(A \cap B)
\end{aligned}
$$

Analogamente, $|A \cup B| \cdot|A \cap B| \leq|A| \cdot|B|$

\subsubsection{PRINCÍPIO DA INCLUSÃO E EXCLUSÃO PARA DOIS EVENTOS}

Seja $\left(\Omega, 2^{\Omega}, P\right)$ um espaço finito de probabilidade, então para $A, B \subset \Omega$

$$
P(A \cup B)=P(A)+P(B)-P(A \cap B)
$$

Demonstração. Como $\mathrm{A} \cup \mathrm{B}=(\mathrm{A} \backslash \mathrm{B}) \cup \mathrm{B}$ e $(\mathrm{A} \backslash \mathrm{B}) \cap \mathrm{B}=\phi$ do terceiro postulado de Kolmogorov,

$\mathrm{P}(\mathrm{A} \cup \mathrm{B})=\mathrm{P}(\mathrm{A} \backslash \mathrm{B})+\mathrm{P}(\mathrm{B})$

Da propriedade $\mathrm{c}), \mathrm{P}(\mathrm{A} \backslash \mathrm{B})=\mathrm{P}(\mathrm{A})-\mathrm{P}(\mathrm{A} \cap \mathrm{B})$, daí:

$$
\begin{aligned}
& \mathrm{P}(\mathrm{A} \cup \mathrm{B})=\mathrm{P}(\mathrm{A})-\mathrm{P}(\mathrm{A} \cap \mathrm{B})+\mathrm{P}(\mathrm{B}) \\
& \mathrm{P}(\mathrm{A} \cup \mathrm{B})=\mathrm{P}(\mathrm{A})+\mathrm{P}(\mathrm{B})-\mathrm{P}(\mathrm{A} \cap \mathrm{B})
\end{aligned}
$$


Outra prova da igualdade $P(A \cup B)+P(A \cap B)=P(A)+P(B)$ é dada pela subtração das igualdades:

$$
\begin{gathered}
P(A \cup B)=P(A)+P(\bar{A} \cap B), \text { pois } A \cap(\bar{A} \cap B)=\phi \\
P(B)=P(A \cap B)+P(\bar{A} \cap B), \text { pois }(A \cap B) \cap(\bar{A} \cap B)=\phi
\end{gathered}
$$

válidas pelo terceiro postulado de Kolmogorov, resulta na fómula $P(A \cup B)+$ $P(A \cap B)=P(A)+P(B)$

\subsubsection{PRINCÍPIO DA INCLUSÃO E EXCLUSÃO PARA TRÊS EVENTOS}

Seja $\left(\Omega, 2^{\Omega}, P\right)$ um espaço finito de probabilidade. Para quaisquer três eventos $A, B, C \subset \Omega$

$\Longrightarrow P(A \cup B \cup C)=P(A)+P(B)+P(C)-P(A \cap B)-P(A \cap C)-P(B \cap C)+P(A \cap B \cap C)$

Demonstração.

$$
P(A \cup B \cup C)=P[A \cup(B \cup C)]
$$

Pelo princípio da inclusão e exclusão válido para dois eventos,

$$
P(A \cup B \cup C)=P(A)+P(B \cup C)-P(A \cap(B \cup C))
$$

Como

$$
P(A \cap(B \cup C))=P[(A \cap B) \cup(A \cap C)]
$$

$\mathrm{e}$

$$
P[(A \cap B) \cup(A \cap C)]=P(A \cap B)+P(A \cap C)-P(A \cap B \cap C)
$$

Ainda pela probpriedade 1.9.1,

$$
P(B \cup C)=P(B)+P(C)-P(B \cap C)
$$

$\log$,

$$
\begin{aligned}
& P(A \cup B \cup C)=P(A)+P(B)+P(C)-P(B \cap C)-[P(A \cap B)+P(A \cap C)-P(A \cap B \cap C)] \\
& P(A \cup B \cup C)=P(A)+P(B)+P(C)-P(A \cap B)-P(A \cap C)-P(B \cap C)+P(A \cap B \cap C)
\end{aligned}
$$


Analogamente, o princípio da inclusão-exclusão é válido para o cálculo do número de elementos da união de dois subconjuntos de um conjunto finito

$$
|A \cup B|=|A|+|B|-|A \cap B|
$$

e para o cálculo de número de elementos da união de três subconjuntos de um conjunto finito

$|A \cup B \cup C|=|A|+|B|+|C|-|A \cap B|-|A \cap C|-|B \cap C|+|A \cap B \cap C|$

\section{Corolário}

a) $P(A \cap B \cap \bar{C})=P(A \cap B)-P(A \cap B \cap C)$

b) $P(A \cap \bar{B} \cap \bar{C})=P(A)-P(A \cap B)-P(A \cap C)+P(A \cap B \cap C)$

c) $P(\bar{A} \cap \bar{B} \cap \bar{C})=P(\overline{A \cup B \cup C})=1-P(A \cup B \cup C)$

\subsubsection{PRINCÍPIO DA INCLUSÃO E EXCLUSÃO PARA N EVENTOS}

Seja $\left(\Omega, 2^{\Omega}, P\right)$ um espaço finito de probabilidade. Se $\forall A_{1}, A_{2}, \ldots A_{n} \subset \Omega$

$$
\begin{aligned}
P\left(A_{1} \cup A_{2} \cup \ldots \cup A_{n}\right) & =\sum_{1 \leq i \leq n} P\left(A_{i}\right) \\
& -\sum_{1 \leq i<j \leq n} P\left(A_{i} \cap A_{j}\right) \\
& +\sum_{1 \leq i<j<k \leq n} P\left(A_{i} \cap A_{j} \cap A_{k}\right)-\ldots+ \\
& +(-1)^{n+1} P\left(A_{1} \cap A_{2} \cap \ldots \cap A_{n}\right)
\end{aligned}
$$

(Prova por indução finita sobre o número de eventos).

Exemplo 1.9.1. Com a hipótese de que todas as cartas tem a mesma chance de figurar em uma mão de bridge, constituída por 13 cartas de um baralho padrão, a probabilidade da mão conter a sequencia $A K Q J$ de copas é igual a

$$
\frac{\left(\begin{array}{c}
48 \\
9
\end{array}\right)}{\left(\begin{array}{c}
52 \\
13
\end{array}\right)}
$$

e a probabilidade de uma mão de bridge conter a sequência $A K Q J$ de pelo menos um dos naipes do baralho é calculada por

$$
P\left(A_{1} \cup A_{2} \cup A_{3} \cup A_{4}\right)
$$


em que $A_{1}, A_{2}, A_{3}, A_{4}$ são os eventos constituídos pelas mãos de bridge com a sequência $A K Q J$ de copas, ouro, paus e espadas respectivamente; como

$$
\begin{gathered}
P\left(A_{i} \cap A_{j}\right)=\frac{\left(\begin{array}{c}
44 \\
5
\end{array}\right)}{\left(\begin{array}{c}
52 \\
13
\end{array}\right)} i, j \in\{1,2,, 3,4\}, \text { com } i<j \\
P\left(A_{i} \cap A_{j} \cap A_{k}\right)=\frac{\left(\begin{array}{c}
40 \\
1
\end{array}\right)}{\left(\begin{array}{c}
52 \\
13
\end{array}\right)} i, j, k \in\{1,2,, 3,4\}, \text { com } i<j, k \\
P\left(A_{1} \cap A_{2} \cap A_{3} \cap A_{4}\right)=0
\end{gathered}
$$

então

$$
\begin{gathered}
P\left(A_{1} \cup A_{2} \cup A_{3} \cup A_{4}\right)= \\
=\left(\begin{array}{l}
4 \\
1
\end{array}\right) \cdot \frac{\left(\begin{array}{c}
48 \\
9
\end{array}\right)}{\left(\begin{array}{c}
52 \\
13
\end{array}\right)}-\left(\begin{array}{l}
4 \\
2
\end{array}\right) \cdot \frac{\left(\begin{array}{c}
44 \\
5
\end{array}\right)}{\left(\begin{array}{l}
52 \\
13
\end{array}\right)}+\left(\begin{array}{l}
4 \\
3
\end{array}\right) \cdot \frac{\left(\begin{array}{c}
40 \\
1
\end{array}\right)}{\left(\begin{array}{c}
52 \\
13
\end{array}\right)} .
\end{gathered}
$$

Em uma mão de bridge de 13 cartas de um baralho padrão, a probabilidade de que pelo menos 9 cartas da mão de 13 cartas são do mesmo naipe é igual a

$$
\frac{\left(\begin{array}{l}
4 \\
1
\end{array}\right)\left(\begin{array}{c}
13 \\
9
\end{array}\right)\left(\begin{array}{c}
39 \\
4
\end{array}\right)+\left(\begin{array}{l}
4 \\
1
\end{array}\right)\left(\begin{array}{l}
13 \\
10
\end{array}\right)\left(\begin{array}{c}
39 \\
3
\end{array}\right)+\left(\begin{array}{l}
4 \\
1
\end{array}\right)\left(\begin{array}{c}
13 \\
13
\end{array}\right)\left(\begin{array}{c}
39 \\
2
\end{array}\right)+\left(\begin{array}{l}
4 \\
1
\end{array}\right)\left(\begin{array}{c}
13 \\
12
\end{array}\right)\left(\begin{array}{c}
39 \\
1
\end{array}\right)+\left(\begin{array}{l}
4 \\
1
\end{array}\right)\left(\begin{array}{c}
13 \\
13
\end{array}\right)\left(\begin{array}{c}
39 \\
0
\end{array}\right)}{\left(\begin{array}{c}
52 \\
13
\end{array}\right)}
$$

Exemplo 1.9.2. Dez cartões numerados com os dígitos $0,1,2,3,4,5,6,7,8,9$ são colocados em uma urna. Um dos cartões é retirado da urna e após a leitura do dígito é recolocada na urna. A operação é repetida um número $k$ de vezes. Com a hipótese de que todos os cartões tem a mesma chance de serem retirados, a probabilidade de que entre os $k$ dígitos retirados

a) não ocorra o dígito zero é

$$
\left(\frac{9}{10}\right)^{k}
$$

b) não ocorra o dígito um é

$$
\left(\frac{9}{10}\right)^{k}
$$

c) não ocorra o dígito zero e nem o dígito um é

$$
\left(\frac{8}{10}\right)^{k}
$$


d) não ocorra o dígito zero ou não ocorra o dígito um é

$$
\left(\frac{9}{10}\right)^{k}+\left(\frac{9}{10}\right)^{k}-\left(\frac{8}{10}\right)^{k}
$$

tendo em vista que o modelo probabilístico é o espaço de probabilidade finito equiprovável $\left(\Omega, 2^{\Omega}, P\right)$ em que $\Omega$ é o conjunto de todas as sequências de comprimento $k$ cujos elementos são os dígitos $0,1,2,3,4,5,6,7,8,9$ e $A$ e $B$ são os eventos constituídos por todas as sequências de comprimento $k$ cujos os elementos são os dígitos $1,2,3,4,5,6,7,8,9$ e $0,2,3,4,5,6,7,8,9$ respectivamente. Então

$$
\begin{gathered}
P(A)=P(B)=\left(\frac{9}{10}\right)^{k} \\
P(A \cap B)=\left(\frac{8}{10}\right)^{k} \\
P(A \cup B)=P(A)+P(B)-P(A \cap B)=2 \cdot\left(\frac{9}{10}\right)^{k}-\left(\frac{8}{10}\right)^{k}
\end{gathered}
$$

\subsubsection{TEOREMA DE WARING PARA DOIS EVENTOS}

Dados dois eventos $A$ e $B$ em um espaço de probabilidade $\left(\Omega, 2^{\Omega}, P\right)$ a probabilidade de ocorrência de exatamente um dos dois eventos $A$ e $B$ é igual a

$$
\begin{aligned}
P(A \Delta B) & =P(A \cap \bar{B})+P(\bar{A} \cap B) \\
& =P(A)+P(B)-2 P(A \cap B) \\
& =P(A)-P(A \cap B)+P(B)-P(A \cap B) \\
& =P(A)+P(B)-2 \cdot P(A \cap B) \\
& =S_{1}^{(2)}-\left(\begin{array}{l}
2 \\
1
\end{array}\right) S_{2}^{(2)}
\end{aligned}
$$

quando $S_{1}^{(2)}$ e $S_{2}^{(2)}$ são definidos por

$$
\begin{aligned}
S_{1}^{(2)} & =P(A)+P(B) \\
S_{2}^{(2)} & =P(A \cap B)
\end{aligned}
$$




\subsubsection{TEOREMA DE WARING PARA TRÊS EVENTOS}

\section{CASO A}

Dados três eventos $A, B$ e $C$ em um espaço de probabilidade $\left(\Omega, 2^{\Omega}, P\right)$, a probabilidade de ocorrência de exatamente um dos três eventos $A, B$ e $C$ é igual a

$$
\begin{gathered}
P(A \cap \bar{B} \cap \bar{C})+P(\bar{A} \cap B \cap \bar{C})+P(\bar{A} \cap \bar{B} \cap C) \\
=P(A)-P(A \cap B)-P(B \cap C)+P(A \cap B \cap C) \\
+P(B)-P(A \cap B)-P(A \cap C)+P(A \cap B \cap C) \\
+P(C)-P(A \cap C)-P(B \cap C)+P(A \cap B \cap C) \\
=P(A)+P(B)+P(C) \\
\quad-\quad 2[P(A \cap B)+P(A \cap C)+P(B \cap C)] \\
+\quad 3 P(A \cap B \cap C) \\
=S_{1}^{(3)}-\left(\begin{array}{l}
2 \\
1
\end{array}\right) S_{2}^{(3)}+\left(\begin{array}{l}
3 \\
1
\end{array}\right) S_{3}^{(3)}
\end{gathered}
$$

quando $S_{1}^{(3)}, S_{2}^{(3)}$ e $S_{3}^{(3)}$ são definidos por

$$
\begin{aligned}
& S_{1}^{(3)}=P(A)+P(B)+P(C) \\
& S_{2}^{(3)}=P(A \cap B)+P(A \cap C)+P(B \cap C) \\
& S_{3}^{(3)}=P(A \cap B \cap C)
\end{aligned}
$$

\section{CASO B}

Dados três eventos $A, B$ e $C$ em um espaço de probabilidade $\left(\Omega, 2^{\Omega}, P\right)$, a probabilidade de ocorrência de exatamente dois dos eventos $A, B$ e $C$ é igual a

$$
\begin{aligned}
& P(A \cap B \cap \bar{C})+P(A \cap \bar{B} \cap C)+P(\bar{A} \cap B \cap C) \\
= & P(A \cap B)-P(A \cap B \cap C) \\
+ & P(A \cap C)-P(A \cap B \cap C) \\
+ & P(B \cap C)-P(A \cap B \cap C) \\
= & P(A \cap B)+P(A \cap C)+P(B \cap C)-3 P(A \cap B \cap C)
\end{aligned}
$$


e pode ser escrito como

$$
=S_{2}^{(3)}-\left(\begin{array}{l}
3 \\
2
\end{array}\right) S_{3}^{(3)}
$$

quando $S_{2}^{(3)}$ e $S_{3}^{(3)}$ são definidos por

$$
\begin{aligned}
S_{2}^{(3)} & =P(A \cap B)+P(A \cap C)+P(B \cap C) \\
S_{3}^{(3)} & =P(A \cap B \cap C)
\end{aligned}
$$

\subsubsection{TEOREMA DE WARING}

Dados $n$ eventos $A_{1}, A_{2}, \ldots, A_{n}$ em um espaço de probabilidade $\left(\Omega, 2^{\Omega}, P\right)$, sejam os números reias $S_{0}, S_{1}, \ldots, S_{n}$ definidos por

$$
\begin{aligned}
& S_{0}^{(n)}=1 \\
& S_{1}^{(n)}=\sum_{i=1}^{n} P\left(A_{i}\right) \\
& S_{2}^{(n)}=\sum_{i=1}^{n} \sum_{j=i+1}^{n} P\left(A_{i} \cap A_{j}\right) \\
& \text {. } \\
& S_{r}^{(n)}=\sum_{j_{1}=1}^{n} \sum_{j_{2}=i+1}^{n} \ldots \sum_{j_{r}=j_{r-1}+1}^{n} P\left(A_{j_{1}} \cap A_{j_{2}} \cap \ldots \cap A_{j_{r}}\right) \\
& \text {. } \\
& S_{n}^{(n)}=P\left(A_{1} \cap A_{2} \cap \ldots \cap A_{n}\right)
\end{aligned}
$$

Para cada $k=1,2, \ldots, n$ a probabilidade do evento $E_{k}$ em que há a ocorrência simultânea de exatamente $k$ dos $n$ eventos $A_{1}, A_{2}, \ldots, A_{n}$ é igual 
a

$$
\begin{aligned}
P\left(E_{k}\right) & =S_{k}^{(n)}-\left(\begin{array}{c}
k+1 \\
k
\end{array}\right) S_{k+1}^{(n)}+\left(\begin{array}{c}
k+2 \\
k
\end{array}\right) S_{k+2}^{(n)}+. .+(-1)^{n-k} S_{n}^{(n)} \\
& =\sum_{j=k}^{n}(-1)^{j-k}\left(\begin{array}{l}
j \\
k
\end{array}\right) S_{j}^{(n)} \\
& =\sum_{j=0}^{n-k}(-1)^{j}\left(\begin{array}{c}
k+j \\
k
\end{array}\right) S_{k+j}^{(n)}
\end{aligned}
$$

A probabilidade do evento $E_{0}$ em que não há a ocorrência de nenhum dos eventos $A_{1}, A_{2}, \ldots, A_{n}$ é igual a

$$
P\left(E_{0}\right)=1-S_{1}^{(n)}+S_{2}^{(n)}-S_{3}^{(n)}+\ldots+(-1)^{n} S_{n}^{(n)}
$$

Generalizando, para cada $m \in\{1,2, \ldots, n\}$ a probabilidade $P_{m}$ de ocorrência de pelo menos $m$ dos $n$ eventos $A_{1}, A_{2}, \ldots, A_{n}$ é igual a

$$
P_{m}=\sum_{j=0}^{n-m}(-1)^{j}\left(\begin{array}{c}
m-1+j \\
m-1
\end{array}\right) S_{m+j}^{(n)}
$$

O princípio da inclusão exclusão em probabilidade para eventos $A_{1}, A_{2}, \ldots, A_{n}$ calcula a probabilidade de ocorrência de pelo menos um dos eventos $A_{1}, A_{2}, \ldots, A_{n}$,

$$
P\left(A_{1} \cup A_{2} \cup \ldots \cup A_{n}\right)=\sum_{j=1}^{n}(-1)^{j-1} S_{j}^{(n)}
$$

Exemplo 1.9.3. O problema dos encontros é o seguinte: dados $N$ urnas numeradas de 1 a $N$ e $N$ bolas numeradas de 1 a $N$, as bolas são colocadas nas urnas de forma aleatória e de modo que haja apenas uma bola em cada urna. Caso o número da bola coincida com o número da urna que a contém, há a ocorrência de um encontro.

A probabilidade de ocorrência de pelo menos um encontro na distribuição aleatória de $N$ bolas numeradas em $N$ urnas numeradas, com a condição de uma única bola em cada urna e com a hipótese de que a chance de uma urna conter uma bola é a mesma para todas as urnas e todas as bolas, é igual a

$$
1-\frac{1}{2 !}+\frac{1}{3 !}-\frac{1}{4 !}+\ldots+(-1)^{N} \frac{1}{N !}
$$

e este valor é aproximadamente igual a

$$
1-\frac{1}{e} \cong 0,63212
$$


para $N$ suficientemente grande.

A probabilidade de ocorrência de exatos $n$ encontros na distribuição aleatória de $N$ bolas numeradas em $N$ urnas numeradas, com a condição de uma única bola em cada urna e com a hipótese de que a chance de uma urna conter uma bola é a mesma para todas as urnas e todas as bolas, é igual a

$$
\frac{1}{n !}\left[1-1+\frac{1}{2 !}-\frac{1}{3 !}+\ldots+(-1)^{N-n} \frac{1}{(N-n) !}\right]
$$

e esse valor é aproximadamente igual a

$$
\frac{1}{n !} \cdot \frac{1}{e}
$$

quando a diferença $(N-n)$ é suficientemente grande.

O modelo probabilístico é o espaço de probabilidade finito equiprovável $\left(\Omega, 2^{\Omega}, P\right)$ cujo espaço amostral $\Omega$ contém todas as $N$ ! sequências $\left(z_{1}, z_{2}, \ldots, z_{N}\right)$ cuja componente $z_{j}$ de número $j$ indica o número da bola colocada na urna de número $j$ para cada $j=1,2, \ldots, N$. O evento $A_{1} \subset \Omega$ contém todas as $(N-1)$ ! sequências cujo primeiro elemento é 1 , o evento $A_{2} \subset \Omega$ contém todas as $(N-1)$ ! sequências cujo segundo elemento é 2 , e assim por diante $A_{N} \subset \Omega$ é o evento que contém todas as $(N-1)$ ! sequências cujo último elemento é N.

Para cada $k=1,2, \ldots, N$ e para cada conjunto de $k$ elementos $\left\{j_{1}, j_{2}, \ldots, j_{k}\right\} \subset$ $\{1,2, \ldots, N\}$ a probabilidade do evento $A_{j_{1}} \cap A_{j_{2}} \cap \ldots \cap A_{j_{k}}$ é igual a

$$
P\left(A_{j_{1}} \cap A_{j_{2}} \cap \ldots \cap A_{j_{k}}\right)=\frac{(N-k) !}{N !}
$$

o que implica que os números definidos no teorema de WARING são iguais a

$$
S_{k}^{(N)}=\left(\begin{array}{c}
N \\
k
\end{array}\right) \frac{(N-k) !}{N !}=\frac{1}{k !}
$$

a probabilidade de ocorrência de exatos $m$ encontros é igual a

$$
\begin{aligned}
\sum_{j=0}^{N-n}(-1)^{j}\left(\begin{array}{c}
n+j \\
n
\end{array}\right) S_{n+j}^{(N)} & =\sum_{j=0}^{N-n}(-1)^{j} \frac{(n+j) !}{n ! j !} \cdot \frac{1}{(n+j) !} \\
& =\frac{1}{n !} \sum_{j=0}^{N-n}(-1)^{j} \frac{1}{j !} \\
& =\frac{1}{n !}\left[1-1+\frac{1}{2 !}-\frac{1}{3 !}+\ldots+(-1)^{N-n} \frac{1}{(N-n) !}\right]
\end{aligned}
$$


Pelo princípio da inclusão-exclusão, a probabilidade de ocorrência de pelo menos um encontro na distribuição aleatória de $N$ bolas numeradas em $N$ urnas numeradas é calculada por

$$
\begin{aligned}
\sum_{j=1}^{N}(-1)^{j} S_{j}^{(N)} & =S_{0}^{(N)}-S_{1}^{(N)}+S_{2}^{(N)}+\ldots+(-1)^{N} S_{N}^{(N)} \\
& =1-\frac{1}{2 !}+\frac{1}{3 !}+\ldots+(-1)^{N} \frac{1}{N !}
\end{aligned}
$$

\subsubsection{DESIGUALDADE DE BOOLE}

\section{Desigualdade de Boole para dois eventos}

A desigualdade de Boole para dois eventos $A$ e $B$ é

$$
P(A \cup B) \leq P(A)+P(B)
$$

de fato, pelo princípio da inclusão-exclusão, para dois eventos

$$
P(A \cup B)=P(A)+P(B)-P(A \cap B)
$$

como $P(A \cap B) \geq 0$,

$$
P(A \cup B) \leq P(A)+P(B)
$$

e, no caso de eventos disjuntos, quando $A \cap B=\phi$

$$
P(A \cup B)=P(A)+P(B)
$$

\section{Desigualdade de Boole para três eventos}

Para três eventos, a desigualdede de Boole é dada em particular

$$
P(A \cup B \cup C) \leq P(A)+P(B)+P(C)
$$

e, no caso de $A \cap B=\phi, A \cap C=\phi$ e $B \cap C=\phi$

$$
P(A \cup B \cup C)=P(A)+P(B)+P(C) .
$$

Demonstração. Como $A \cup B \cup C$ é expresso como união de eventos disjuntos como

$$
A \cup B \cup C=A \cup(B \backslash A) \cup(C \backslash(A \cup B))
$$




$$
P(A \cup B \cup C)=P[A \cup(B \backslash A) \cup(C \backslash(A \cup B))]
$$

como $A \cap(B \backslash A)=\phi, A \cap(C \backslash(A \cup B))=\phi$ e $(B \backslash A) \cap(C \backslash(A \cup B))=\phi$ pelo terceiro postulado de Kolmogorov,

$$
P(A \cup B \cup C)=P(A)+P(B \backslash A)+P[C \backslash(A \cup B)]
$$

como $(B \backslash A) \subset B, P(B \backslash A) \leq P(B)$

e

$[C \backslash(A \cup B)] \subset C, P[C \backslash(A \cup B)] \leq P(C)$,

$$
P(A \cup B \cup C) \leq P(A)+P(B)+P(C)
$$

No caso de $A \cap B=\phi, A \cap C=\phi$ e $B \cap C=\phi$ a demonstração é trivial.

\section{Desigualdade de Boole para $\mathbf{n}$ eventos}

Dada uma sequência finita de eventos $A_{1}, A_{2}, \ldots, A_{n}$ em um espaço finito de probabilidade $\left(\Omega, 2^{\Omega}, P\right)$. Então

$$
P\left(\bigcup_{i}^{n} A_{i}\right) \leq \sum_{i}^{n} P\left(A_{i}\right)
$$

e no caso de $A_{i} \cap A_{j}=\phi \operatorname{com} i, \mathrm{j} \in\{1,2, \ldots, n\}$ e $i \neq j$

$$
P\left(\bigcup_{i}^{n} A_{i}\right)=\sum_{i}^{n} P\left(A_{i}\right)
$$

Por indução finita é possível provar a desigualdade de Boole no caso de uma sequência finita de eventos.

\subsubsection{DESIGUALDADE DE BONFERRONI}

Dada uma sequência finita de eventos $A_{1}, A_{2}, \ldots, A_{n}$ em um espaço finito de probabilidade $\left(\Omega, 2^{\Omega}, P\right)$. Então

$$
P\left(\bigcap_{i=1}^{n} A_{i}\right) \geq P(\Omega)-\sum_{i=1}^{n}\left(P(\Omega)-P\left(A_{i}\right)\right)=1-n+\sum_{i=1}^{n} P\left(A_{i}\right)
$$


Demonstração. Da desigualdade de Boole,

$$
\begin{gathered}
P\left(\bigcup_{i}^{n} \overline{A_{i}}\right) \leq \sum_{i}^{n} P\left(\overline{A_{i}}\right) \\
P\left(\overline{A_{1}} \cup \ldots \cup \overline{A_{n}}\right) \leq P\left(\overline{A_{1}}\right)+\ldots+P\left(\overline{A_{n}}\right)
\end{gathered}
$$

Utilizando a Lei De Morgan e também a propriedade que $P(\bar{A})=1-$ $P(A)$,

$$
\begin{aligned}
& P\left(\overline{A_{1} \cap A_{2} \cap \ldots \cap A_{n}}\right) \leq 1-P\left(A_{1}\right)+1-P\left(A_{2}\right)+\ldots+1-P\left(A_{n}\right) \\
& 1-P\left(A_{1} \cap A_{2} \cap \ldots \cap A_{n}\right) \leq 1-P\left(A_{1}\right)+1-P\left(A_{2}\right)+\ldots+1-P\left(A_{n}\right) \\
& 1-P\left(A_{1} \cap A_{2} \cap \ldots \cap A_{n}\right) \leq \sum_{i=1}^{n}\left(P(\Omega)-P\left(A_{i}\right)\right. \\
& P\left(A_{1} \cap A_{2} \cap \ldots \cap A_{n}\right) \geq 1-\sum_{i=1}^{n}\left(P(\Omega)-P\left(A_{i}\right)\right. \\
& P\left(\bigcap_{i=1}^{n} A_{i}\right) \geq P(\Omega)-\sum_{i=1}^{n}\left(P(\Omega)-P\left(A_{i}\right)=1-\sum_{i=1}^{n}\left[1-P\left(A_{i}\right)\right]=1-n+\sum_{i=1}^{n} P\left(A_{i}\right)\right.
\end{aligned}
$$

\subsection{PROBABILIDADE CONDICIONAL}

Quando uma pessoa visita uma família com três filhos, a probabilidade de que essa família tenha exatos duas filhas é igual a $\frac{3}{8}$ que diminui para o valor de $\frac{1}{4}$ no momento em que o filho mais velho da família abre a porta da casa e, nesse momento, a probabilidade dessa família possuir três filhas passa a ser zero, enquanto que a probabilidade deste primogênito ter pelo menos um irmão do sexo masculino é igual a $\frac{3}{4}$.

Ao ter a informação que um homem tinha dois periquitos de estimação e que pelo menos um dos dois periquitos é macho, a probabilidade do outro periquito também ser macho, não é $\frac{1}{2}$ e sim $\frac{1}{3}$ visto que a possibilidade de duas periquitas fêmeas estar descartada. No caso de dois periquitos: um de cor verde e outro de cor amarela, a informação de que o periquito verde é macho indica que a probabilidade do outro periquito ser macho sobe a $\frac{2}{4}$ 
pois apenas duas situações agora ocorrem: o verde é macho e o amarelo é macho e o verde é macho e o preto é fêmea.

Um senhor dá uma festa em sua casa. Ao término da festa, ele descobre que sua coleção de quadros foi furtada. Após as investigações, o detetive tem certeza que o ladrão foi uma das 90 pessoas presentes à festa e que dessas pessoas 48 eram do sexo feminino. O detetive encontra na cena do crime alguns pertences do ladrão que indicam que o ladrão é do sexo masculino. Dado que o senhor Ricardo foi o primeiro suspeito do sexo masculino a ser interrogado, a probabilidade de que ele seja o ladrão é de $\frac{1}{42}$ e não $\frac{1}{90}$.

O problema de Monty Hall é um jogo em que um participante tem que escolher uma entre três portas, sendo que uma delas tem um carro como prêmio e as outras duas estão vazias. O apresentador do jogo pede ao participante que escolha uma porta. Se o participante escolher a porta que tem o carro, ele ganha o carro. Entretanto, após o participante escolher uma porta, o apresentador abre uma outra porta do jogo e mostra que aquela porta está vazia. O apresentador, então, pergunta ao participante se ele quer mudar de porta. Há apenas duas portas sobrando, aquela que o participante escolheu primeiro e aquela que não foi aberta. Qual é a probabilidade do participante ganhar o prêmio, se mudar de porta?

De maneira geral, as pessoas acham que é $50 \%$, pois o prêmio está na porta que você escolheu, ou está na outra porta que sobrou. Mas essa resposta não é correta.

Para calcular a probabilidade do participante ganhar o prêmio se mudar de porta, logo após o apresentador abrir uma das portas em que o prêmio não está. Nomeando as portas, teremos: PORTA A (não está com o carro), PORTA B (não esta com o carro) e PORTA CERTA (está com o carro). Analisando as situações:

1) A probabilidade do participante escolher a PORTA A é $\frac{1}{3}$, feito essa escolha, o apresentador irá abrir a PORTA B e o participante trocará para a PORTA CERTA, ganhando o carro.

2) A probabilidade do participante escolher a PORTA B é $\frac{1}{3}$, com essa escolha o apresentador abrirá a PORTA A e o participante trocaria para a PORTA CERTA, também ganhando o prêmio.

3) A probabilidade do participante escolher a PORTA CERTA é $\frac{1}{3}$, sendo assim o apresentador irá abrir a PORTA A ou a PORTA B e o participante trocaria a sua escolha para a porta que o apresentador deixou de abrir (PORTA A ou PORTA B) perdendo o prêmio.

Logo, o participante ganhará o prêmio se escolher inicialmente a PORTA 
A ou a PORTA B e a probabilidade de que isso ocorra é $\frac{1}{3}+\frac{1}{3}=\frac{2}{3}$ e não $\frac{1}{2}$ como geralmente as pessoas acham.

O conceito de probabilidade condicional de um evento com a certeza da ocorrência de um outro evento é agora introduzido após a descrição das situações apresentadas.

Definição 1.10.1. Em um espaço de probabilidade $\left(\Omega, 2^{\Omega}, P\right)$ a probabilidade $P(A \mid B)$ do evento $A$ condicionada a ocorrência do evento $B$, desde que $P(B)>0$, é definida por

$$
P(A \mid B)=\frac{P(A \cap B)}{P(B)}
$$

\section{Propriedade}

Se $P(A) \cdot P(B) \neq 0$

$$
\begin{aligned}
P(A \mid B) & =\frac{P(A \cap B)}{P(B)} \\
& =\frac{P(B \cap A)}{P(A)} \cdot \frac{P(A)}{P(B)} \\
& =P(B \mid A) \cdot \frac{P(A)}{P(B)}
\end{aligned}
$$

o que implica:

$$
\frac{P(A \mid B)}{P(A)}=\frac{P(B \mid A)}{P(B)}
$$

e que se

$$
P(A \mid B)>P(A) \Longrightarrow P(B \mid A)>P(B) .
$$

Exemplo 1.10.2. O sangue humano está classificado em quatro grupos distintos: A, B, AB e O. Além disso, o sangue de uma pessoa pode possuir, ou não, o fator Rhésus. Se o sangue de uma pessoa possui esse fator, diz-se que a pessoa pertence ao grupo sanguíneo Rhésus positivo $\left(R h^{+}\right)$e, se não possui esse fator, diz-se Rhésus negativo $\left(R h^{-}\right)$. Numa pesquisa, 1000 pessoas foram classificadas, segundo grupo sanguíneo e respectivo fator Rhésus, de acordo com a tabela.

\begin{tabular}{|c|c|c|c|c|}
\hline & $\mathrm{A}$ & $\mathrm{B}$ & $\mathrm{AB}$ & $\mathrm{O}$ \\
\hline$R h^{+}$ & 390 & 60 & 50 & 350 \\
\hline$R h^{-}$ & 70 & 20 & 10 & 50 \\
\hline
\end{tabular}

Dentre as 1000 pessoas pesquisadas, escolhida uma ao acaso 
a) a probabilidade de seu grupo sanguíneo ser $\mathrm{AB}$ e $R h^{+}$é

$$
P\left((A B) \cap R h^{+}\right)=\frac{50}{1000}=\frac{1}{20} .
$$

b) a probabilidade condicional de ser $\mathrm{AB}$, sabendo-se que a pessoa escolhida é $R h^{+}$

A probabilidade condicional de ser $\mathrm{AB}$, sabendo que a pessoa escolhida é $R h^{+}$é

$$
P\left((A B) \mid R h^{+}\right)=\frac{P\left((A B) \cap R h^{+}\right)}{P\left(R h^{+}\right)}=\frac{\frac{50}{1000}}{\frac{850}{1000}}=\frac{1}{17}
$$

Exemplo 1.10.3. Uma urna contém 8 boals brancas e 4 bolas pretas. O experimento aleatório consiste na repetição de quatro vezes do seguinte processo: retirada de uma bola cuja cor é anotada e a bola extraída é recolocada na urna.

Com a hipótese de que todas as bolas tem a mesma chance de serem escolhidas, e com o conhecimento prévio de que extamente três bolas brancas foram selecionadas, a probabilidade condicional de que a primeira e a terceira bolas selecionadas sejam brancas é $\frac{1}{2}$.

O modelo probabilístico definido pelo espaço finito não equiprovável de probabilidade $\left(\Omega, 2^{\Omega}, P\right)$, cujo espaço amostral $\Omega$ é dado por

$$
\Omega=\{(i, j, k, l): i, j, k, l \in\{0,1\}\}
$$

em que $(1,1,0,1)$ indica que a primeira, a segunda e a quarta bola são pretas e a terceira bola é branca e a função de probabilidade $P$ calculada no subconjunto unitário $\{(1,1,0,1)\}$ é igual a

$$
P(\{(1,1,0,1)\})=\frac{4}{12} \cdot \frac{4}{12} \cdot \frac{8}{12} \cdot \frac{4}{12}
$$

Os eventos $A, B$ e $A \cap B$ que traduzem respectivamente ocorrência de bola branca na primeira e na terceira retirada, ocorrência de exatamente três bolas brancas e ocorrência de exatamente três bolas brancas com bolas brancas ocupando a primeira e a terceira posições, são definidos por

$$
\begin{aligned}
& A=\{(0, i, 0, j): i, j \in\{0,1\}\} ; \\
& B=\{(0,0,0,1),(0,0,1,0),(0,1,0,0),(1,0,0,0)\} ;
\end{aligned}
$$




$$
\begin{aligned}
A \cap B= & (0,0,0,1),(0,1,0,0)\} \\
P(B)= & P(\{(0,0,0,1),(0,0,1,0),(0,1,0,0),(1,0,0,0)\}) \\
= & \frac{8}{12} \cdot \frac{8}{12} \cdot \frac{8}{12} \cdot \frac{4}{12}+\frac{8}{12} \cdot \frac{8}{12} \cdot \frac{4}{12} \cdot \frac{8}{12}+\frac{8}{12} \cdot \frac{4}{12} \cdot \frac{8}{12} \cdot \frac{8}{12}+\frac{4}{12} \cdot \frac{8}{12} \cdot \frac{8}{12} \cdot \frac{8}{12} \\
= & 4 \cdot\left(\frac{8}{12} \cdot \frac{8}{12} \cdot \frac{8}{12} \cdot \frac{4}{12}\right) \\
& P(A \cap B)= \\
= & \frac{8}{12} \cdot \frac{8}{12} \cdot \frac{8}{12} \cdot \frac{4}{12}+\frac{8}{12} \cdot \frac{4}{12} \cdot \frac{8}{12} \cdot \frac{8}{12} \\
= & 2 \cdot\left(\frac{8}{12} \cdot \frac{8}{12} \cdot \frac{8}{12} \cdot \frac{4}{12}\right)
\end{aligned}
$$

A probabilidade condicional procurada é

$$
\begin{aligned}
P(A \mid B) & =\frac{P(A \cap B)}{P(B)} \\
& =\frac{2 \cdot\left(\frac{8}{12} \cdot \frac{8}{12} \cdot \frac{8}{12} \cdot \frac{4}{12}\right)}{4 \cdot\left(\frac{8}{12} \cdot \frac{8}{12} \cdot \frac{8}{12} \cdot \frac{4}{12}\right)} \\
& =\frac{1}{2}
\end{aligned}
$$

\subsection{FÓRMULA SEQUENCIAL DE BAYES}

A fórmula equivalente da definição de probabilidade condicional

$$
P(A \cap B)=P(B) \cdot P(A \mid B)
$$

tem como generalização a fórmula sequencial de Bayes: dada uma sequência finita de eventos $A_{1}, A_{2}, \ldots, A_{n}$ em um espaço de probabilidade $\left(\Omega, 2^{\Omega}, P\right)$,

$P\left(A_{1} \cap A_{2} \cap \ldots \cap A_{n}\right)=P\left(A_{1}\right) \cdot P\left(A_{2} \mid A_{1}\right) \cdot P\left(A_{3} \mid A_{1} \cap A_{2}\right) \cdot \ldots \cdot P\left(A_{n} \mid A_{1} \cap A_{2} \cap \ldots \cap A_{n-1}\right)$ ou definindo para cada $k=1,2, \ldots, n$ 


$$
\begin{gathered}
B_{k}=A_{1} \cap A_{2} \cap \ldots \cap A_{k} \\
P\left(B_{n}\right)=P\left(B_{1}\right) \prod_{k=2}^{n} P\left(B_{k} \mid B_{k-1}\right)
\end{gathered}
$$

Demonstração. Observando que para $k=2, \ldots, n$

$$
\begin{gathered}
B_{k} \subset B_{k-1} \\
P\left(B_{k} \mid B_{k-1}\right)=\frac{P\left(B_{k}\right)}{P\left(B_{k-1}\right)}
\end{gathered}
$$

a fórmula

$$
P\left(B_{n}\right)=P\left(B_{1}\right) \prod_{k=2}^{n} P\left(B_{k} \mid B_{k-1}\right)=P\left(B_{n}\right)
$$

é provada.

Exemplo 1.11.1. Uma urna contém 7 bolas azuis, 6 bolas brancas e 5 bolas vermelhas. Três bolas são retiradas em sequência uma a uma da urna sem devolução na urna da bola extraída a cada passo. Com a hipótese de que todas as bolas tenham a mesma chance de serem retiradas da urna, a probabilidade de que a primeira bola extraída seja vermelha, a segunda branca e a terceira azul tem com resultado óbivio

$$
\frac{5}{18} \cdot \frac{6}{17} \cdot \frac{7}{16}=\frac{35}{816}
$$

mas o cálculo da probabilidade requerida será dado segundo o modelo probabilístico definido pelo espaço finito não equiprovável de probabilidade $\left(\Omega, 2^{\Omega}, P\right)$, cujo espaço amostral $\Omega$ é dado por

$$
\Omega=\{(i, j, k): i, j, j \in\{0,1,2\}\}
$$

em que $(0,1,2)$ indica que a primeira bola retirada é azul, a segunda branca e a terceira vermelha e a função de probabilidade $P$ calculada no subconjunto unitário $\{(0,1,2)\}$.

Os eventos $A, B$ e $C$ são respectivamente iguais a

$$
\begin{aligned}
& \mathrm{A}=\{(2, i, j): i, j \in\{0,1,2\}\} \\
& \mathrm{B}=\{(i, 1, j): i, j \in\{0,1,2\}\}
\end{aligned}
$$




$$
\mathrm{C}=\{(i, j, 0): i, j \in\{0,1,2\}\}
$$

cujas probabilidades são calculadas por

$$
P(\{(0,1,2)\})=\frac{7}{18} \cdot \frac{6}{17} \cdot \frac{5}{16}
$$

e no subconjunto unitário $\{(0,0,2)\}$

$$
\begin{gathered}
P(\{(0,0,2)\})=\frac{7}{18} \cdot \frac{6}{17} \cdot \frac{5}{16} \\
P(A)=P(\{(2,0,0)\})+P(\{(2,0,1)\})+P(\{(2,0,2)\}) \\
+\quad P(\{(2,1,0)\})+P(\{(2,1,1)\})+P(\{(2,1,2)\}) \\
+\quad P(\{(2,2,0)\})+P(\{(2,2,1)\})+P(\{(2,2,2)\}) \\
=\frac{5 \cdot 7 \cdot 6+5 \cdot 7 \cdot 6+5 \cdot 7 \cdot 4+5 \cdot 6 \cdot 7+5 \cdot 6 \cdot 5+5 \cdot 6 \cdot 4+5 \cdot 4 \cdot 7+5 \cdot 4 \cdot 6+5 \cdot 4 \cdot 3}{18 \cdot 17 \cdot 16} \\
=\frac{1360}{4896} \\
=\frac{5}{18}=\frac{5 \cdot 6 \cdot 7}{18 \cdot 17 \cdot 16} \cdot \frac{5 \cdot 6 \cdot 5}{18 \cdot 17 \cdot 16} \cdot \frac{5 \cdot 6 \cdot 4}{18 \cdot 17 \cdot 16} \\
\text { Como } A \cap B=\{(2,1, j): j \in\{0,1,2\}\} \\
P(A \cap B)=P(\{2,1,0)\})+P(\{(2,1,1)\})+P(\{(2,1,2)\}) \\
\text { Como } A \cap B \cap C=\{(2,1,0)\} \quad P(A \cap B \cap C)=P(\{(2,1,0)\}) \\
=\frac{5 \cdot 6 \cdot 7}{18 \cdot 17 \cdot 16}
\end{gathered}
$$

que é a probabilidade procurada.

Com o conhecimento prévio de que a primeira bola é vermelha, a probabilidade de que a segunda é branca é a probabilidade condicional

$$
P(B \mid A)=\frac{P(A \cap B)}{P(A)}
$$

Sabendo que a primeira bola é vermelha e a segunda é branca, a probabilidade de que a terceira bola é azul é a probabilidade condicional

$$
P(C \mid A \cap B)=\frac{P(A \cap B \cap C)}{P(A \cap B)}=\frac{\frac{5 \cdot 6 \cdot 7}{18 \cdot 17 \cdot 16}}{\frac{5 \cdot 6 \cdot 7}{18 \cdot 17 \cdot 16} \cdot \frac{5 \cdot 6 \cdot 5}{18 \cdot 17 \cdot 16} \cdot \frac{5 \cdot 6 \cdot 4}{18 \cdot 17 \cdot 16}}
$$




$$
\begin{aligned}
P(A \cap B \cap C) & =P(A) \cdot P(B \mid A) \cdot P(C \mid A \cap B) \\
& =\frac{35}{816}
\end{aligned}
$$

Exemplo 1.11.2. Uma urna contém inicialmente 5 bolas brancas e 7 bolas pretas. O experimento aleatório consiste na repetição de quatro vezes do seguinte processo: retirada de uma bola da urna cuja cor é anotada e a bola extaída é recolocada na urna junto com mais duas bolas de mesma cor.

Com a hipótese de que todas as bolas tem a mesma chance de serem escolhidas, a probabilidade a ser calculada é a probabilidade de que as duas primeiras bolas selecionadas sejam pretas e as duas bolas seguintes sejam brancas.

O modelo probabilístico é o espaço finito de probabilidade não equiprovável $\left(\Omega, 2^{\Omega}, P\right)$ cujo espaço amostral $\Omega$ é definido por

$$
\Omega=\{(i, j, k, l): i, j, k, l \in\{0,1\}\}
$$

em que $(1,0,1,1)$ indica que a primeira, terceira e quarta bola são pretas e a segunda bola é branca e a função de probabilidade $P$ calculada no subconjunto unitário $\{(1,0,1,1)\}$ é igual a

$$
P(\{(1,0,1,1)\})=\frac{7}{12} \cdot \frac{5}{14} \cdot \frac{9}{16} \cdot \frac{11}{18}
$$

Os eventos $A, B, C, D$, que traduzem respectivamente a ocorrência de bola cor preta na primeira retirada, a ocorrência de bola cor preta na segunda retirada, a ocorrência de bola cor branca na terceira retirada e a ocorrência de bola cor branca na quarta retirada, são definidos por

$$
\begin{aligned}
& \mathrm{A}=\{(1, i, j, k): i, j, k \in\{0,1\}\} \\
& \mathrm{B}=\{(i, 1, j, k): i, j, k \in\{0,1\}\} \\
& \mathrm{C}=\{(i, j, 0, k): i, j, k \in\{0,1\}\} \\
& \mathrm{D}=\{(i, j, k, 0): i, j, k \in\{0,1\}\}
\end{aligned}
$$

A probabilidade pedida é

$$
\begin{aligned}
P(A \cap B \cap C \cap D) & =\frac{7}{12} \cdot \frac{7+2}{12+2} \cdot \frac{5}{12+2+2} \cdot \frac{5+2}{12+2+2+2} \\
& =\frac{7}{12} \cdot \frac{9}{14} \cdot \frac{5}{16} \cdot \frac{7}{18} \\
& =\frac{35}{768} .
\end{aligned}
$$


A probabilidade procurada também pode ser calculada como

$P(A \cap B \cap C \cap D)=P(A) \cdot P(B \mid A) \cdot P(C \mid A \cap B) \cdot P(D \mid A \cap B \cap C)$

Para o cálculo de probabilidade condicional

$$
P(D \mid A \cap B \cap C)=\frac{P(A \cap B \cap C \cap D)}{P(A \cap B \cap C)}
$$

basta o cálculo de

$$
\begin{aligned}
P(A \cap B \cap C) & =P(\{(1,1,0, i): i \in\{0,1\}\}) \\
& =P(\{(1,1,0,0)\})+P(\{(1,1,0,1)\}) \\
& =\frac{7}{12} \cdot \frac{7+2}{12+2} \cdot \frac{5}{12+2+2} \cdot \frac{5+2}{12+2+2+2} \\
& +\frac{7}{12} \cdot \frac{7+2}{12+2} \cdot \frac{5}{12+2+2} \cdot \frac{9}{12+2+2+2} \\
& =\frac{5}{48}
\end{aligned}
$$

e assim

$$
P(D \mid A \cap B \cap C)=\frac{\frac{35}{768}}{\frac{5}{48}}=\frac{7}{16}
$$

\subsection{TEOREMA DE BAYES}

Dada uma partição finita com $\mathrm{n}$ subconjuntos $B_{1}, B_{2}, \ldots, B_{n}$ do espaço amostral $\Omega$ de um espaço finito de probabilidade $\left(\Omega, 2^{\Omega}, P\right)$, isto é, $\Omega=B_{1} \cup, B_{2} \cup \ldots \cup B_{n}$ e $B_{i} \cap B_{j}=\phi$ para $i, \mathrm{j} \in\{1,2, \ldots, n\}$ com $i \neq j$.

Para cada evento $A$,

$$
P(A)=P\left(A \mid B_{1}\right) \cdot P\left(B_{1}\right)+P\left(A \mid B_{2}\right) \cdot P\left(B_{2}\right)+\ldots+P\left(A \mid B_{n}\right) \cdot P\left(B_{n}\right)
$$

Demonstração. Como

$$
A=A \cap \Omega=A \cap\left(\bigcup_{j=1}^{n} B_{j}\right)=\bigcup_{j=1}^{n}\left(A \cap B_{j}\right)
$$

Pelo terceiro postulado de Kolmogorov 


$$
P(A)=\sum_{j=1}^{n} P\left(A \cap B_{j}\right)
$$

se $P\left(B_{j}\right)=0$, então $P\left(A \cap B_{j}\right)=0$ desde que $A \cap B_{j} \subset B_{j}$

se $P\left(B_{j}\right)>0$, então $P\left(A \cap B_{j}\right)=P\left(A \mid B_{j}\right) \cdot P\left(B_{j}\right)$, o que completa a demonstração do teorema de Bayes.

Exemplo 1.12.1. Uma empresa possui três fornecedores (A, B e C) de uma determinada peça. O fornecedor A é responsável por 30\% do fornecimento, o fornecedor B é responsável por $45 \%$ do fornecimento, e o restante vem do fornecedor C. Cada um dos fornecedores, fornecem produtos que são considerados "defeituosos" e correspondem a $1 \%, 2 \%$ e $1,5 \%$, respectivamente, dos totais fornecidos. Qual é a probabilidade de encontrar um produto defeituoso durante a inspeção de qualidade?

Seja o evento $\mathrm{D}=$ \{produto com defeito $\}, \mathrm{A}=$ \{produto fornecido pelo fornecedor $\mathrm{A}\}, \mathrm{B}=\{$ produto fornecido pelo fornecedor $\mathrm{B}\}$ e $\mathrm{C}=$ \{produto fornecido pelo fornecedor $\mathrm{C}\}$. Do enunciado, temos que

$P(A)=0,3, P(B)=0,45$ e $P(C)=0,25$

e ainda $P(D \mid A)=0,3, P(D \mid B)=0,45$ e $P(D \mid C)=0,25$.

Pelo teorema de Bayes, temos que a probabilidade de encontrar um produto com defeito durante uma inspeção de qualidade é

$$
\begin{aligned}
& P(D)=P(D \mid A) \cdot P(A)+P(D \mid B) \cdot P(B)+P(D \mid C) \cdot P(C) \\
& P(D)=0,01 \cdot 0,3+0,02 \cdot 0,45+0,015 \cdot 0,25 \\
& P(D)=0,01575=1,575 \%
\end{aligned}
$$

\subsection{TEOREMA DE BAYES PARA PROBABILI- DADE CONDICIONAL}

Dada uma partição finita com $n$ subconjuntos não vazios $B_{1}, B_{2}, B_{3}, \ldots, B_{n}$ do espaço amostral $\Omega$ de um espaço finito de probabilidade $\left(\Omega, 2^{\Omega}, P\right)$, isto é,

$$
\Omega=B_{1} \cup B_{2} \cup B_{3} \cup \ldots \cup B_{n}
$$

e

$$
B_{i} \cap B_{j}=\phi \operatorname{com} i, \mathrm{j} \in\{1,2,3, \ldots, n\} \text { e } i \neq j
$$

Com a hipótese de que $P\left(B_{j}\right) \geq 0$ para cada $j=1,2,3, \ldots, n$ para cada evento $A$ e para cada $j=1,2,3, \ldots, n$. 


$$
P\left(B_{j} \mid A\right)=\frac{P\left(A \mid B_{j}\right) \cdot P\left(B_{j}\right)}{P\left(A \mid B_{1}\right) \cdot P\left(B_{1}\right)+P\left(A \mid B_{2}\right) \cdot P\left(B_{2}\right)+\ldots+P\left(A \mid B_{n}\right) \cdot P\left(B_{n}\right)}
$$

Demonstração. Sejam os eventos $B_{1}, B_{2}, \ldots, B_{n}$ uma partição de $\Omega$, ou seja, $B_{1} \cap B_{2} \cap \ldots \cap B_{n}=\phi$ e $B_{1} \cup B_{2} \cup \ldots \cup B_{n}=\Omega$ e seja A um evento não vazio do espaço amostral $\Omega$.

Da definição de probabilidade condicional,

$$
\begin{gathered}
P\left(A \mid B_{j}\right)=\frac{P\left(A \cap B_{j}\right)}{P\left(B_{j}\right)} \\
\text { ou } P\left(A \cap B_{j}\right)=P\left(A \mid B_{j}\right) \cdot P\left(B_{j}\right) \\
\text { Como } A=\left(A \cap B_{1}\right) \cup\left(A \cap B_{2}\right) \cup \ldots \cup\left(A \cap B_{n}\right) \text { é uma união disjunta, } \\
P(A)=P\left(A \mid B_{1}\right) \cdot P\left(B_{1}\right)+P\left(A \mid B_{2}\right) \cdot P\left(B_{2}\right)+\ldots+P\left(A \mid B_{n}\right) \cdot P\left(B_{n}\right)
\end{gathered}
$$

Pela definição de probabilidade condicional,

$$
\begin{gathered}
P\left(B_{j} \mid A\right)=\frac{P\left(B_{j} \cap A\right)}{P(A)} \\
P\left(B_{j} \mid A\right)=\frac{P\left(A \mid B_{j}\right) \cdot P\left(B_{j}\right)}{P\left(A \mid B_{1}\right) \cdot P\left(B_{1}\right)+P\left(A \mid B_{2}\right) \cdot P\left(B_{2}\right)+\ldots+P\left(A \mid B_{n}\right) \cdot P\left(B_{n}\right)}
\end{gathered}
$$

Exemplo 1.13.1. "O doente sadio e o sadio doente"(MAGALHÃES, 2011, p.33)

Os testes de laboratórios para detectar doenças envolvem vários procedimentos e para avaliar a eficiência de um teste é necessário quantificar a probabilidade de erro. Vamos denominar falso-positivo para o erro que o teste indica positivo para um paciente que não tem a doença e denotaremos falso-negativo se o teste não acusar a doença para um paciente doente. Considere que um determinado teste resulta positivo para um paciente não doentes, com probabilidade 0,1 e que a probabilidade do teste ser negativo para um paciente com a doença também seja 0,1 . Vamos supor que a incidência da doença na população seja de 1 para cada 10.000 habitante. Qual a probabilidade de uma pessoa estar realmente doente se o teste deu positivo? 
Definindo os eventos:

$D$ : a pessoa está doente $\Longrightarrow \bar{D}$ : a pessoa não está doente;

$A$ : o teste é positivo $\Longrightarrow \bar{A}$ : o teste é negativo.

Do enunciado, temos: $P(D)=\frac{1}{10.000}=0,0001 ; P(A \mid \bar{D})=0,1 \mathrm{e}$ $P(\bar{A} \mid D)=0,1$.

Utilizando a propriedade de eventos complementares, temos: $P(\bar{D})=$ 0,9999 e $P(A \mid D)=0,9$.

Queremos calcular a probabilidade de uma pessoa estar realmente doente sabendo que o teste deu positiva, ou seja queremos calcular $P(D \mid A)$, pelo teorema de Bayes, temos:

$$
\begin{aligned}
P(D \mid A) & =\frac{P(A \mid D) P(D)}{P((A \mid D) P(D)+P(A \mid \bar{D}) P(\bar{D})} \\
& =\frac{0,9 \cdot 0,0001}{0,9 \cdot 0,0001+0,1 \cdot 0,9999} \\
& =0,0009
\end{aligned}
$$

Logo, a probabilidade de uma pessoa estar realmete doente, sabendo que o teste deu positivo é 0,0009 .

Exemplo 1.13.2. Três moedas estão em uma caixa: uma delas possui duas caras, a segunda é uma moeda não viciada com chances iguais para ocorrência de cara e coroa e a terceira delas é uma moeda viciada cujo resultdo é cara em $75 \%$ dos lançamentos.

Com a hipótese de que as três moedas tem a mesma chance de serem retiradas de dentro da caixa, o experimento aleatório consiste na seleção aleatória de uma das moedas.

Para a obtenção da probabilidade de ocorrência da moeda de duas caras com o conhecimento antecipado de que o resultado do lançamento é cara, o modelo probabilístico é o espaço finito de probabilidade não equiprovável $\left(\Omega, 2^{\Omega}, P\right)$, cujo espaço amostral $\Omega$ é definido por

$$
\Omega=\{(i, j): i \in\{0,1,2\} j \in\{0,1\}\}
$$

em que $(2,0)$ indica a seleção da moeda viciada e no lançamento o resultado foi cara, $(1,1)$ indica a seleção da moeda honesta com resultado no lançamento de coroa e $(0,0)$ indica a seleção da moeda de duas caras com resultado cara no lançamento. 
A função de probabilidade $P$ é definida por

$$
\begin{aligned}
P(\{(0,1)\}) & =0 \\
P(\{(0,0)\}) & =\frac{1}{3} \\
P(\{(1,0)\}) & =\frac{1}{3} \cdot \frac{1}{2}=P(\{(1,1)\}) \\
P(\{(2,0)\}) & =\frac{1}{3} \cdot \frac{3}{4} \\
P(\{(2,1)\}) & =\frac{1}{3} \cdot \frac{1}{4}
\end{aligned}
$$

Considerando os eventos:

$$
\begin{aligned}
& A=\{(i, 0): i \in\{0,1,2\}\} \\
& E_{1}=\{(0, j): j \in\{0,1\}\} \\
& E_{2}=\{(1, j): j \in\{0,1\}\} \\
& E_{3}=\{(2, j): j \in\{0,1\}\}
\end{aligned}
$$

tem-se

$$
\begin{gathered}
P\left(E_{1}\right)=P\left(E_{2}\right)=P\left(E_{3}\right)=\frac{1}{3} \\
P\left(A \mid E_{1}\right)=\frac{P\left(A \cap E_{1}\right)}{P\left(E_{1}\right)}=\frac{P(\{(0,0)\}}{P\left(E_{1}\right)}=1 \\
P\left(A \mid E_{2}\right)=\frac{P\left(A \cap E_{2}\right)}{P\left(E_{2}\right)}=\frac{P(\{(1,0)\}}{P\left(E_{2}\right)}=\frac{1}{2} \\
P\left(A \mid E_{3}\right)=\frac{P\left(A \cap E_{3}\right)}{P\left(E_{3}\right)}=\frac{P(\{(2,0)\}}{P\left(E_{3}\right)}=\frac{3}{4}
\end{gathered}
$$

A probabilidade de ocorrência da moeda de duas caras quando o resultado do lançamento é cara é a probabilidade condicional

$$
\begin{aligned}
P\left(E_{1} \mid A\right) & =\frac{P\left(A \mid E_{1}\right) \cdot P\left(E_{1}\right)}{P\left(A \mid E_{1}\right) \cdot P\left(E_{1}\right)+P\left(A \mid E_{2}\right) \cdot P\left(E_{2}\right)+P\left(A \mid E_{3}\right) \cdot P\left(E_{3}\right)} \\
& =\frac{1 \cdot \frac{1}{3}}{1 \cdot \frac{1}{3}+\frac{1}{2} \cdot \frac{1}{3}+\frac{3}{4} \cdot \frac{1}{3}} \\
& =\frac{4}{9} .
\end{aligned}
$$

calculada pelo teorema de Bayes. 


\subsection{EVENTOS INDEPENDENTES}

Definição 1.14.1. Dois eventos $A$ e $B$ em um espaço de probabilidade $(\Omega, F, P)$ são ditos eventos independentes em relação a função de probabilidade $P$ se e somente se:

$$
P(A \cap B)=P(A) \cdot P(B)
$$

o que é equivalente a

$$
P(A \mid B)=P(A) \text { desde que } P(B)>0
$$

e a

$$
P(B \mid A)=P(B) \text { desde que } P(A)>0
$$

No caso de eventos $A$ e $B$ não necessariamente independentes

$$
P(A \cap B)=P(A)+P(B)-P(A \cup B) \leq P(A)+P(B) .
$$

No caso de eventos $A$ e $B$ independentes

$$
\begin{aligned}
P(A \cup B) & =P(A)+P(B)-P(A) \cdot P(B) \\
P(A \cup \bar{B}) & =P(A)+P(\bar{B})-P(A) \cdot P(\bar{B}) \\
& =P(A)+1-P(B)-P(A)[1-P(B)] \\
P(A \cap \bar{B}) & =P(A)-P(A \cap B) \\
& =P(A)[1-P(B)] \\
& =P(A) \cdot P(\bar{B})
\end{aligned}
$$

\subsubsection{DEFINIÇÃO}

Três eventos $A, B$ e $C$ em um espaço de probabilidade $(\Omega, F, P)$ em relação a função de probabilidade $P$ são ditos eventos independentes se e somente se:

i) $P(A \cap B \cap C)=P(A) \cdot P(B) \cdot P(C)$

ii) $P(A \cap B)=P(A) \cdot P(B)$

iii) $P(A \cap C)=P(A) \cdot P(C)$

iv) $P(B \cap C)=P(B) \cdot P(C)$ 
No caso de eventos $A, B$ e $C$ não necessariamente independentes

$$
\begin{aligned}
P(A \cap B \cap C) & =P(\overline{\bar{A} \cup \bar{B} \cup \bar{C}}) \\
& =1-P(\bar{A} \cup \bar{B} \cup \bar{C}) \\
& =1-[P(\bar{A})+P(\bar{B})+P(\bar{C})] \\
& +[P(\bar{A} \cap \bar{B})+P(\bar{A} \cap \bar{C})+P(\bar{B} \cap \bar{C})] \\
& -P(\bar{A} \cap \bar{B} \cap \bar{C})
\end{aligned}
$$

\subsubsection{PROPRIEDADES}

P1) Se $A$ e $B$ são eventos independentes em relação a mesma função $P$ de probabilidade, então em relação a $P$

i) $A$ e $\bar{B}$ são eventos independentes

ii) $\bar{A}$ e $\bar{B}$ são eventos independentes

Demonstração. Como (ii) é consequência de (i), basta provar que $P(A \cap \bar{B})=$ $P(A) \cdot P(\bar{B})=P(A) \cdot[1-P(B)]$ de fato, como

$$
A=(A \cap B) \cup(A \cap \bar{B}) \text { e }(A \cap B) \cap(A \cap \bar{B})=\phi
$$

Pelo terceiro axioma de Kolmogorov, temos:

$$
\begin{gathered}
P(A)=P(A \cap B)+P(A \cap \bar{B}) \\
P(A)=P(A) \cdot P(B)+P(A \cap \bar{B})
\end{gathered}
$$

$\mathrm{e}$

$$
P(A \cap \bar{B})=P(A) \cdot P(\bar{B})=P(A) \cdot[1-P(B)]
$$

P2) Se $A, B$ e $C$ são três eventos independentes em relação a mesma função $P$ de probabilidade então, em relação a $P$,

i) $\bar{A}, B$ e $C$ são eventos independentes

ii) $\bar{A}, \bar{B}$ e $C$ são eventos independentes

iii) $\bar{A}, \bar{B}$ e $\bar{C}$ são eventos independentes 
Demonstração. Para a prova do item (i), resta provar que

$$
P(\bar{A} \cap B \cap C)=P(\bar{A}) \cdot P(B) \cdot P(C)=[1-P(A)] \cdot P(B) \cdot P(C)
$$

Como $B \cap C=(B \cap C \cap A) \cup(B \cap C \cap \bar{A})$

Pelo terceiro axioma de Kolmogorov, temos:

$$
P(B \cap C)=P(A \cap B \cap C)+P(\bar{A} \cap B \cap C)
$$

Por hipótese,

$$
P(B) \cdot P(C)=P(A) \cdot P(B) \cdot P(C)+P(\bar{A} \cap B \cap C)
$$

$\mathrm{e}$

$$
P(\bar{A} \cap B \cap C)=[1-P(A)] \cdot P(B) \cdot P(C)
$$

P3) Se os eventos $A, B$ e $C$ em um espaço de probabilidade $(\Omega, F, P)$ de tal modo que $A$ e $B$ são eventos independentes e $A$ e $C$ também são independentes, então

a) se $C \subset B$, os eventos $A$ e $B \backslash C$ são eventos independentes.

b) se $B \cap C=\phi$, os eventos $A$ e $B \cup C$ são eventos independentes.

Demonstração. item a)

$$
\begin{aligned}
P(A \cap(B \backslash C)) & =P(A \cap B \cap \bar{C}) \\
& =P[(A \cap B) \backslash(A \cap B \cap C)] \\
& =P(A \cap B)-P(A \cap B \cap C) \\
& =P(A \cap B)-P(A \cap C), \text { desde que } C \subset B \\
& =P(A) P(B)-P(A) P(C) \\
& =P(A)[P(B)-P(C)] \\
& =P(A) P(B \backslash C)
\end{aligned}
$$


Demonstração. item b)

$$
\begin{aligned}
P(A \cap(B \cup C)) & =P[(A \cap B) \cup(A \cap C)] \\
& =P(A \cap B)+P(A \cap C), \text { desde que } B \cap C=\phi \\
& =P(A) P(B)+P(A) P(C) \\
& =P(A)[P(B)+P(C)] \\
& =P(A) P(B \cup C), \text { desde que } B \cap C=\phi
\end{aligned}
$$

P4) Dados os eventos independentes $A, B$ e $C$ em um espaço de probabilidade $(\Omega, F, P)$, então os eventos

a) $A$ e $B \cup C$ são eventos independentes.

b) $A$ e $B \backslash C$ são eventos independentes.

Demonstração. item a)

$$
\begin{aligned}
P[A \cap(B \cup C)] & =P[A \backslash A \cap \overline{(B \cup C)}] \\
& =P(A)-P(A \cap \bar{B} \cap \bar{C}) \\
& =P(A)-P(A) P(\bar{B}) P(\bar{C}) \\
& =P(A)[1-P(\bar{B}) P(\bar{C})] \\
& =P(A)[1-P(\bar{B} \cap \bar{C})] \\
& =P(A)[1-P(\overline{B \cup C})] \\
& =P(A) P(B \cup C)
\end{aligned}
$$

Demonstração. item b)

$$
\begin{aligned}
P(A \cap(B \backslash C)) & =P(A \cap B \cap \bar{C}) \\
& =P(A) P(B) P(\bar{C}) \\
& =P(A) P(B \cap \bar{C}) \\
& =P(A) P(B \backslash C)
\end{aligned}
$$


No caso de três eventos $A, B, C$ independentes

$$
\begin{aligned}
& P(A \cup B \cup C)=P(A)+P(B)+P(C) \\
&-P(A) P(B)-P(A) P(C)-P(B) P(C) \\
&+P(A) P(B) P(C) \\
& P(A \cup \bar{B} \cup \bar{C})=P(A)+P(\bar{B})+P(\bar{C}) \\
&-P(A) P(\bar{B})-P(A) P(\bar{C})-P(B) P(\bar{C}) \\
&+P(A) P(\bar{B}) P(\bar{C}) \\
& \\
& P(A \cap \bar{B} \cap \bar{C})=P(A) \cdot P(\bar{B}) \cdot P(\bar{C}) \\
& \quad \\
& P(A \cup B \cup \bar{C})=P(A)+P(B)+P(\bar{C}) \\
&+\quad P(A) P(\bar{B}) P(\bar{C}) \\
& P(A \cap B \cap \bar{C})=P(A) \cdot P(B) \cdot P(\bar{C}) \\
&
\end{aligned}
$$

P5) Dadas duas sequências de eventos

$$
A_{1}, A_{2}, \ldots, A_{m}
$$

e

$$
B_{1}, B_{2}, \ldots, B_{n}
$$

mutuamente exclusivos, isto é,

$$
A_{i} \cap A_{j}=\phi, \operatorname{com} i, \mathrm{j} \in\{1,2, \ldots, m\} \text { e } i \neq j
$$

e

$$
B_{i} \cap B_{j}=\phi, \operatorname{com} i, \mathrm{j} \in\{1,2, \ldots, n\} \text { e } i \neq j
$$

de um espaço de probabilidade $(\Omega, F, P)$ com a propriedade de que, para cada escolha de índices $i$ e $j$, em relação a função $P$ de probabilidade $A_{i}$ e $B_{j}$ são eventos independentes. 
Então os eventos

$$
A=\bigcup_{i=1}^{m} A_{i} \text { e } B=\bigcup_{j=1}^{n} B_{j}
$$

são eventos independentes em relação a $P$.

P6) Dados $n \geq 1$ eventos independentes $A_{1}, A_{2}, \ldots, A_{n}$ em um espaço de probabilidade $(\Omega, F, P)$, a probabilidade de que

a) nenhum dos eventos $A_{1}, A_{2}, \ldots, A_{n}$ ocorra é igual a

$$
\begin{aligned}
P\left(\overline{A_{1}} \cap \overline{A_{2}} \cap \ldots \cap \overline{A_{n}}\right) & =P\left(\overline{A_{1}}\right) \cdot P\left(\overline{A_{2}}\right) \cdot \ldots \cdot P\left(\overline{A_{n}}\right) \\
& =\left[1-P\left(A_{1}\right)\right] \cdot\left[1-P\left(A_{2}\right)\right] \cdot \ldots \cdot\left[1-P\left(A_{n}\right)\right]
\end{aligned}
$$

b) ao menos um dos eventos $A_{1}, A_{2}, \ldots, A_{n}$ ocorra é igual

$$
\begin{aligned}
P\left(A_{1} \cup A_{2} \cup \ldots \cup A_{n}\right) & =1-P\left(\overline{A_{1}} \cap \overline{A_{2}} \cap \ldots \cap \overline{A_{n}}\right) \\
& =1-\left[1-P\left(A_{1}\right)\right] \cdot\left[1-P\left(A_{2}\right)\right] \cdot \ldots \cdot\left[1-P\left(A_{n}\right)\right]
\end{aligned}
$$

c) exatamente um dos eventos $A_{1}, A_{2}, \ldots, A_{n}$ ocorra é igual

$$
\begin{aligned}
P\left(A_{1} \cap \overline{A_{2}} \cap \ldots \cap \overline{A_{n}}\right) & +P\left(\overline{A_{1}} \cap A_{2} \cap \ldots \cap \overline{A_{n}}\right)+\ldots+P\left(\overline{A_{1}} \cap \overline{A_{2}} \cap \ldots \cap A_{n}\right)= \\
& =P\left(\overline{A_{1}}\right) \cdot P\left(\overline{A_{2}}\right) \cdot \ldots \cdot P\left(\overline{A_{n}}\right) \\
& =\left[\frac{P\left(A_{1}\right)}{P\left(\overline{A_{1}}\right)}+\frac{P\left(A_{2}\right)}{P\left(\overline{A_{2}}\right)}+\ldots+\frac{P\left(A_{n}\right)}{P\left(\overline{A_{n}}\right)}\right]
\end{aligned}
$$

Teorema 1.14.2. Se $A_{1}, A_{2}, \ldots, A_{n}$ são eventos independentes, então os eventos

i) $B_{1}, \ldots, B_{n}$, em que $B_{i}$ é $A_{i}$ ou $\overline{A_{i}}, i=1, \ldots, n$, são independente.

ii) $C_{1}, \ldots, C_{k}$, em que $k \leq n$ e $C_{1}, \ldots, C_{k}$ são formados pela união ou interseção de subconjuntos disjuntos de $A_{1}, \ldots, A_{n}$ também são independentes.

Vamos provar apenas o item i) para $\mathrm{n}=2$ e o item ii) para $\mathrm{k}=2$. As provas generalizadas para n e k são mais difíceis e as notações ficariam complicadas. 
Demonstração. Item i)

É suficiente provar i) no caso especial em que $B_{1}=A_{1}$ e $B_{2}=\overline{A_{2}}$.

Se $B_{1}=A_{1}$ e $B_{2}=\overline{A_{2}}$, então

$$
\begin{array}{cc}
B_{1} \cap B_{2}=A_{1} \cap \overline{A_{2}} \\
\Longrightarrow \quad B_{1} \cap B_{2}=A_{1} \cap\left(\Omega-A_{2}\right) \\
\Longrightarrow \quad B_{1} \cap B_{2}=A_{1} \cap \Omega-\left(A_{1} \cap A_{2}\right) \\
\Longrightarrow \quad B_{1} \cap B_{2}=A_{1}-\left(A_{1} \cap A_{2}\right) .
\end{array}
$$

Logo,

$$
\begin{aligned}
P\left(B_{1} \cap B_{2}\right) & =P\left(A_{1}-\left(A_{1} \cap A_{2}\right)\right) \\
& =P\left(A_{1}\right)-P\left(A_{1} \cap A_{2}\right) \\
& \left.=P\left(A_{1}\right)-P\left(A_{1}\right) P\left(A_{2}\right)\right) \\
& =P\left(A_{1}\right)\left[1-P\left(A_{2}\right)\right] \\
& =P\left(A_{1}\right) P\left(\overline{A_{2}}\right) \\
& =P\left(B_{1}\right) P\left(B_{2}\right)
\end{aligned}
$$

Demonstração. Item ii)

Primeiramente vamos mostrar para um caso especial em que tanto $C_{1}$ quanto $C_{2}$ são formados por interseções dos subconjuntos disjuntos de $A_{1}, \ldots, A_{n}$. Neste caso, podemos supor que

$$
C_{1}=\bigcap_{i=1}^{r} A_{i} \text { e } C_{2}=\bigcap_{i=t}^{n} A_{i}
$$

em que $1 \leq r \leq t \leq n$. Neste caso, temos:

$$
\begin{aligned}
P\left(C_{1} \cap C_{2}\right) & =P\left(A_{1} \cap \ldots \cap A_{r} \cap A_{t} \cap \ldots \cap A_{n}\right) \\
& =\left[\prod_{i=1}^{r} P\left(A_{i}\right)\right]\left[\prod_{i=t}^{n} P\left(A_{i}\right)\right] \\
& =P\left(C_{1}\right) P\left(C_{2}\right)
\end{aligned}
$$

Sendo $C_{1}$ definido como no caso acima e $C_{2}=\bigcup_{i}^{n} A_{i}$. Sendo $B_{i}=A_{i}$, i $=$ $1, \ldots$, r e $B_{i}=\overline{A_{i}}, \mathrm{i}=\mathrm{r}+1, \ldots, \mathrm{n}$. Então por i) os eventos $B=1, B_{2}, \ldots, B_{n}$ são independentes, logo 


$$
D_{1}=\bigcap_{i=1}^{r} B_{i} \text { e } D_{2}=\bigcap_{i=t}^{n} B_{i}
$$

Finalmente, se $D_{1}$ e $D_{2}$ são independentes, tome $C_{1}=D_{1}$ e $C_{2}=\overline{D_{2}}$, e novamente por i) os eventos são independentes. Os casos restantes podem ser manipulados similarmente para completar a prova.

Teorema 1.14.3. Para $i=1, \ldots, n$, sendo $A_{i} \subset S_{i}$ e seja $B_{i}$ o evento em que $A_{i}$ ocorre na i-ésima vez. Então $P\left(B_{i}\right)=P\left(A_{i}\right), i=1, \ldots, n$ e $B_{1}, \ldots, B_{n}$ são mutuamente independentes.

Demonstração. Em primeiro lugar vamos mostrar que $P\left(B_{i}\right)=P\left(A_{i}\right)$. Para cada i, temos

$$
B_{i}=S_{1} \ldots S_{i-1} \cdot A_{i} \cdot S_{i+1} \ldots S_{n}
$$

Portanto

$$
P\left(B_{i}\right)=P\left(A_{i}\right) \prod_{j \neq i} P\left(S_{j}\right)=P\left(A_{i}\right)
$$

como afirmado.

Na segunda parte, vamos estabelecer que $B_{1}, B_{2}, \ldots, B_{n}$ são mutuamente independentes. Seja $J$ um subconjunto de $\{1,2, \ldots, n\}$. Então podemos escrever

$$
\bigcap_{i \in J} B_{i}=C_{1} \cdot C_{2} \ldots \ldots C_{n},
$$

em que $C_{i}=A_{i}$ se $i \in J$ e $C_{i}=S_{i}$, caso contrário. Portanto

$$
P\left(\bigcap_{i \in J} B_{i}\right)=\prod_{i=1}^{n} P\left(C_{i}\right)=\prod_{i \in J} P\left(A_{i}\right)=\prod_{i \in J} P\left(B_{i}\right)
$$

Exemplo 1.14.4. Considere o nosso alfabeto que tem no total 26 letras. Dentre todas as palavras formadas com três letras escolhemos uma delas ao acaso. Definimos os eventos:

- A: palavra escolhida começa por s;

- B: a palavra escolhida tem s no meio;

- C: palavra escolhida tem exatamente duas letras iguais. 
Observe que $|\Omega|=26 \cdot 26 \cdot 26=26^{3}$. O evento $A$ possui todas as palavras que começam por s, então $|A|=1 \cdot 26 \cdot 26=26^{2}$, o evento $B$ são todas as palavras que possuem a letra s na posição central, $\operatorname{logo}|B|=26 \cdot 1 \cdot 26=26^{2}$ e o evento $C$ são as palavras que possuem exatamente duas letras iguais, então $|C|=3 \cdot(26 \cdot 1 \cdot 25)=26 \cdot 75$. Clculando as probabilidades dos eventos A, B e C, temos:

$$
\begin{gathered}
P(A)=\frac{26^{2}}{26^{3}} \Longrightarrow P(A)=\frac{1}{26} \\
P(B)=\frac{26^{2}}{26^{3}} \Longrightarrow P(B)=\frac{1}{26} \\
P(C)=\frac{26 \cdot 75}{26^{3}} \Longrightarrow P(C)=\frac{75}{26^{2}}
\end{gathered}
$$

Agora iremos calcular as probabilidades das intersecções dois a dois dos eventos:

O evento $A \cap B$ são palavres que começam por s e que possuem a letra do meio igual a s também, $\operatorname{logo}|A \cap B|=1 \cdot 1 \cdot 26=26$, já o vento $A \cap C$ são as palavras que começam por s e possuem exatamente duas letras iguais, ou seja, as palavras podem ter duas letras iguais a s, sendo que uma delas seria a primeira letra ou a palavra possui duas letras iguais que não são a letra s, mas a primeira letra tem que ser s, então $|A \cap C|=2 \cdot(1 \cdot 1 \cdot 25)+(1 \cdot 25 \cdot 1)=75$, o $|B \cap C|=75$, pois só teríamos apenas que alterar a letra s da primeira para a segunda. O evento $A \cap B \cap C$ são as palavras que começam por s, a letra do meio é igual a s e possui exatamente duas letras iguais, então $|A \cap B \cap C|=1 \cdot 1 \cdot 25=25$. A probabilidades das intersecções são:

$$
\begin{gathered}
P(A \cap B)=\frac{26}{26^{3}} \Longrightarrow P(A \cap B)=\frac{1}{26^{2}} \\
P(A \cap C)=\frac{75}{26^{3}} \\
P(B \cap C)=\frac{75}{26^{3}} \\
P(A \cap B \cap C)=\frac{25}{26^{3}}
\end{gathered}
$$

Verificando a independência dois a dois entre os eventos, teremos: 
- $P(A) \cdot P(B)=\frac{1}{26} \cdot \frac{1}{26}=\frac{1}{26^{2}}=P(A \cap B) \Longrightarrow A$ e $B$ são eventos independentes.

- $P(A) \cdot P(C)=\frac{1}{26} \cdot \frac{75}{26^{2}}=\frac{75}{26^{3}}=P(A \cap C) \Longrightarrow A$ e $C$ são eventos independentes.

- $P(B) \cdot P(C)=\frac{1}{26} \cdot \frac{75}{26^{2}}=\frac{75}{26^{3}}=P(B \cap C) \Longrightarrow B$ e $C$ são eventos independentes.

Entretanto, verificamos que o mesmo não ocorre com a independência dos eventos $A, B$ e $C$, verificamos que

$$
P(A) \cdot P(B) \cdot P(C)=\frac{1}{26} \cdot \frac{1}{26} \cdot \frac{75}{26}=\frac{75}{26^{3}} \neq P(A \cap B \cap C),
$$

logo não há independência entre os eventos $A, B$ e $C$, o que nos leva a concluir que mesmo que ocorra independência dois a dois entre os eventos, não implica independência entre os três eventos.

\subsection{ESPAÇO PRODUTO CARTESIANO DE DOIS EPAÇOS DE PROBABILIDADE}

Dados dois espaços finitos de probabilidade $\left(X, 2^{X}, P_{X}\right)$ e $\left(Y, 2^{Y}, P_{Y}\right)$, o espaço finito de probabilidade produto cartesiano de dois espaços de probabilidade dados e ordenados é a terna ordenada

$$
\left(X \times Y, 2^{X \times Y}, P\right)
$$

cujo espaço amostral $X \mathrm{x} Y$ é o produto cartesiano dos espaços amostrais $X$ e $Y$ correspondentes de cada espaço de probabilidade e cuja função de probabilidade $P$ é definida nos subconjuntos unitários $\{(x, y)\}$ de $X \times Y Y$ em que $x \in X$ e $y \in Y$ por

$$
P(\{(x, y)\})=P_{X}(\{x\}) \cdot P_{Y}(\{y\})
$$

No caso de espaços finitos equiprováveis de probabilidades, o espaço de probabilidade produto cartesiano de espaços equiprováveis de probabilidade, também é um espaço finito equiprovável de probabilidade. A definição de probabilidade, produto cartesiano de três ou mais espaços finitos de probabilidade, é análogo e não será explicitada apesar da utilização no decorrer do trabalho. 


\subsection{PROBABILIDADES BINOMIAIS}

\subsubsection{TEOREMA DAS PROBABILIDADES BINOMIAIS}

Dada uma sequência finita de $n \geq 1$ eventos independentes $A_{1}, A_{2}, \ldots, A_{n}$ de um espaço de probabilidades $\left(\Omega, 2^{\Omega}, P\right)$, cujas probabilidades $P\left(A_{1}\right)=$ $P\left(A_{2}\right)=\ldots=P\left(A_{n}\right)=p$.

Então a probabilidade de que

a) exatamente $k$ dos eventos $A_{1}, A_{2}, \ldots, A_{n}$ ocorrem é dado por

$$
b(k, n, p)=\left(\begin{array}{l}
n \\
k
\end{array}\right) p^{k}(1-p)^{n-k}
$$

b) no mínimo $k$ dos eventos $A_{1}, A_{2}, \ldots, A_{n}$ ocorrem é dado por

$$
\sum_{j=k}^{n} b(j, n, p)
$$

c) no máximo $k$ eventos $A_{1}, A_{2}, \ldots, A_{n}$ ocorrem é dado por

$$
\sum_{j=0}^{k} b(j, n, p)
$$

Demonstração.

a) Para cada subconjunto não vazio $J$, com $J \subset\{1,2, \ldots, n\}$ e $|J|=k$, então

$$
B_{J}=\left(\bigcap_{j \in J} A_{j}\right) \cap\left(\bigcap_{j \notin J} \overline{A_{j}}\right)
$$

é o evento cuja probabilidade é dada por

$$
P\left(B_{J}\right)=\prod_{j \in J} P\left(A_{j}\right) \prod_{j \notin J} P\left(\overline{A_{j}}\right)
$$

pela independência dos eventos $A_{1}, A_{2}, \ldots, A_{n}$

$$
P\left(B_{J}\right)=p^{k}(1-p)^{n-k}
$$

A probabilidade a ser calculada é a probabilidade do evento

$$
E_{k}=\bigcup_{|J|=k} B_{J}
$$


que pelo terceiro axioma de Kolmogorov é igual a

$$
\begin{aligned}
& P\left(E_{k}\right)=\sum_{|J|=k} P\left(B_{J}\right) \\
& =\left(\begin{array}{l}
n \\
k
\end{array}\right) p^{k}(1-p)^{n-k}
\end{aligned}
$$

desde que existem $\left(\begin{array}{l}n \\ k\end{array}\right)$ subconjuntos com k elementos do conjunto $\{1,2, \ldots, n\}$.

Exemplo 1.16.1. Considere uma amostra de 10 bolas retiradas de uma urna que contém 12 bolas vermelhas e 18 verdes. Essa amostra será retirada da seguinte maneira: retira a primeira bola, a cor é anotada e a bola é devolvida para a urna, ai então retira a segunda bola, a cor também é anotada e a bola é devolvida para a urna e assim sucessivamente. Qual é a probabilidade de se retirar 6 bola vermelhas e 4 bolas verdes?

A probabilidade de ser retirada uma bola vermelha é $p=\frac{2}{5}$, o número de bolas vermelhas da amostra deve ser $k=6$ e o número de bolas da amostra é $n=10$, então pelo teorema das probabilidas binomioais, teremos

$$
b\left(6,10, \frac{2}{5}\right)=\left(\begin{array}{c}
10 \\
6
\end{array}\right) \cdot\left(\frac{2}{5}\right)^{6} \cdot\left(\frac{3}{5}\right)^{4} \cong 0,111
$$

Exemplo 1.16.2. Um jogador de basquete acerta $80 \%$ dos lances livres que aremessa. Qual é a probabilidade de que ele acerte 3 arremessos livres em 5 arremessos?

A probabilidade de que o jogador acerte é $p=\frac{4}{5}$, o número de acertos deve ser $k=7$ e o número de arremessos é $n=5$, então pelo teorema das probabilidades binomiais, teremos

$$
b\left(3,5, \frac{4}{5}\right)=\left(\begin{array}{l}
5 \\
3
\end{array}\right) \cdot\left(\frac{4}{5}\right)^{3} \cdot\left(\frac{1}{5}\right)^{2} \cong 0,205
$$




\subsubsection{TEOREMA DAS PROBABILIDADES MULTINO- MIAIS}

Dada uma sequência finita de $n \geq 2$ eventos $A_{1}, A_{2}, \ldots, A_{n}$ de um espaço finito de probabilidade $\left(\Omega, 2^{\Omega}, P\right)$ com as propriedades

a) $A_{i} \cap A_{j}=\phi, i, j \in\{1,2, \ldots, n\}$ e $i \neq j$

b) $A_{1} \cup A_{2} \cup \ldots \cup A_{r}=\Omega$

Para cada $n \in \mathbb{N}=\{1,2,3, \ldots\}$ sejam $n_{1}, n_{2}, \ldots, n_{r} \in \mathbb{N}$ tais que $n_{1}+n_{2}+$ $\ldots+n_{r}=n$. Então a probabilidade de que, em $n$ tentativas independentes, da ocorrência do evento $A_{1}$ exatamente $n_{1}$ vezes, da ocorrência do evento $A_{2}$ exatamente $n_{2}$ vezes, assim por diante até a ocorrência do evento $A_{r}$ exatamente $n_{r}$ vezes é igual a

$$
\frac{n !}{n_{1} ! n_{2} ! \ldots n_{r} !} P\left(A_{1}\right)^{n_{1}} P\left(A_{2}\right)^{n_{2}} \ldots P\left(A_{r}\right)^{n_{r}}
$$

Demonstração. Para espaço finito de probabilidade.

Considerando no espaço produto

$$
(\Omega \times \Omega \times \ldots \times \Omega, \rho(\Omega \times \Omega \times \ldots \times \Omega), P \times P \times \ldots \times P)
$$

em que o espaço amostral $\Omega \times \Omega \times \ldots \times$ é o produto cartesiano de $\Omega n$ vezes, o evento $A_{i j}$ em que a ocorrência de $A_{i}$ acontece na tentativa número $j$ para $i=1,2, \ldots, r$ e $j=1,2, \ldots, n$, isto é, $A_{i j}$ é o produto cartesiano de $(n-1)$ cópias de $\Omega$ e do evento $A_{i}$ na j-ésima possição no produto cartesiano e para cada $\wedge=\left(\wedge_{1}, \wedge_{2}, \ldots, \wedge_{r}\right)$ partição do conjunto $\{1,2, \ldots, n\}$, seja o evento

$$
B_{\wedge}=\bigcap_{1=1}^{r} \bigcap_{j \in r_{i}} A_{i j} \subset \Omega \times \Omega \times \ldots \times \Omega
$$

cuja probabilidade é dada por

$$
\begin{aligned}
(P \times P \times \ldots \times P) B_{\wedge} & =\prod_{i=1}^{r} \prod_{j \in \wedge_{i}}(P \times P \times \ldots \times P)\left(A_{i j}\right) \\
& =\prod_{i=1}^{r} P\left(A_{i}\right)^{n_{i}}
\end{aligned}
$$

em que $n_{i}$ é o número de elementos de $\wedge_{i}$ para $i=1,2, \ldots, r$. 
A probabilidade a ser considerada é a probabilidade do evento $E$ dada por

$$
E=\bigcup_{\wedge} B_{\wedge}
$$

em que a união é tomada sobre todas as partições $\wedge=\left(\wedge_{1}, \wedge_{2}, \ldots, \wedge_{r}\right)$ do conjunto $\{1,2, \ldots, n\}$ de tal maneira que $n_{i}$ é o número de elementos de $\wedge_{i}$ para $i=1,2, \ldots, r$

$$
(P \times P \times \ldots \times P)(E)=\frac{n !}{n_{1} ! n_{2} ! \ldots n_{r} !} P\left(A_{1}\right)^{n_{1}} P\left(A_{2}\right)^{n_{2}} \ldots P\left(A_{r}\right)^{n_{r}}
$$

devido a existência de $\frac{n !}{n_{1} ! n_{2} ! \ldots n_{r} !}$ partições $\wedge$ com propriedades acima e devido ao terceiro axioma de Kolmogorov pois a união dos eventos $\bigcup_{\wedge} B_{\wedge}$ é constituída de eventos dois a dois disjuntos.

Exemplo 1.16.3. Considere uma amostra de sete bolas retiradas de uma urna que contém 12 bolas vermelhas, 16 bolas azuis e 18 verdes. Essa amostra será retirada da seguinte maneira: retira a primeira bola, a cor é anotada e a bola é devolvida para a urna, ai então retira a segunda bola, a cor também é anotada e a bola é devolvida para a urna e assim sucessivamente até a retirada da sétima bola. Determine a probabilidade de que 3 bolas vermelhas, 2 bolas azuis e 2 bolas verdes sejam sacadas.

Considere os eventos $A_{1}, A_{2}$ e $A_{3}$ de um espaço finito de probabilidade $\left(\Omega, 2^{\Omega}, P\right)$, em que

- $A_{1}$ : ocorrência de bola vermelha, com $P\left(A_{1}\right)=\frac{6}{23}$

- $A_{2}$ : ocorrência de bola azul, com $P\left(A_{2}\right)=\frac{8}{23}$

- $A_{3}$ : ocorrência de bola verde com $P\left(A_{3}\right)=\frac{9}{23}$

em sete retiradas independentes, sendo exatamente $n_{1}=3$ ocorrênias do evento $A_{1}, n_{2}=2$ ocorrências do evento $A_{2}$ e $n_{3}=2$ ocorrências do evento $A_{3}$. Então utilizando o teorema das probabilidades multinomiais, a probabilidade de que 3 bolas vermelhas, 2 bolas azuis e 2 bolas verdes sejam sacadas, é dada por

$$
\frac{7 !}{3 ! \cdot 2 ! \cdot 2 !} \cdot\left(\frac{6}{23}\right)^{3} \cdot\left(\frac{8}{23}\right)^{2} \cdot\left(\frac{9}{23}\right)^{2} \cong 0,07
$$




\subsubsection{TEOREMA DAS PROBABILIDADES BINOMIAIS NEGATIVAS}

Seja o evento $A$ em um espaço finito de probabilidade $\left(\Omega, 2^{\Omega}, P\right)$ e, para cada $i=1,2, \ldots, n$, seja o evento $A_{i}$ no espaço produto cartesiano de probabilidade

$$
\left(\Omega \times \Omega \times \ldots \times \Omega, 2^{\Omega \times} \Omega \times \ldots \times \Omega, P \times P \times \ldots \times P\right)
$$

em que o evento $A$ ocorre na tentativa de número $i$, isto é, $A_{i}$ é o produto cartesiano de $(n-1)$ cópias de $\Omega$ com o evento $A$ na i-ésima posição do produto cartesiano cuja probabilidade é igual a

$$
(P \times P \times \ldots \times P)\left(A_{i}\right)=P(A) \text { para } i=1,2, \ldots, n .
$$

Então a probabilidade do evento $A$ ocorrer pela primeira vez na tentativa de número $k, k=1,2, \ldots, n$ é dada pela probabilidade do evento $B_{k}$

$$
b_{k}=\overline{A_{1}} \cap \overline{A_{2}} \cap \ldots \cap \overline{A_{k-1}} \cap A_{k}
$$

calculado por

$$
(P \times P \times \ldots \times P)\left(B_{k}\right)=[1-P(A)]^{k-1} P(A)
$$

Dados $r \in \mathbb{N}, k \in \mathbb{N}, n \in \mathbb{N}$, com $1 \leq r \leq k \leq n$, a probabilidade da ocorrência de $A$ pela r-ésima vez na tentativa de número $k$ é igual a

$$
a(k, r, p)=\left(\begin{array}{c}
k-1 \\
r-1
\end{array}\right) p^{r}(1-p)^{k-r}
$$

$\operatorname{com} p=P(A)$.

Demonstração. Considerando no espaço produto cartesiano de probabilidade

$$
\left.\left(\Omega \times \Omega \times \ldots \times \Omega, 2^{(\Omega \times \Omega \times \ldots \times} \Omega\right), P \times P \times \ldots \times P\right)
$$

os eventos $A_{1}, A_{2}, \ldots, A_{k-1}, A_{k}$ tem a mesma probabilidade $p=P(A)$.

A ocorrência do evento $A$ pela $r$-ésima vez na tentativa de número $k$ é equivalente a ocorrência de $A_{k}$ e que os eventos $A_{1}, A_{2}, \ldots, A_{k-1}$ ocorrem exatas $(r-1)$ vezes. Seja $B$ o evento em que exatas $(r-1)$ dos eventos $A_{1}, A_{2}, \ldots, A_{k}$ ocorrem. Então

$$
\begin{gathered}
(P \times P \times \ldots \times P)(B)=b(r-1, k-1, p) \\
=\left(\begin{array}{c}
k-1 \\
r-1
\end{array}\right) p^{r-1}(1-p)^{k-r}
\end{gathered}
$$

pelo teorema das distribuições binomiais. 
Exemplo 1.16.4. Considere uma amostra de bolas retiradas de uma urna que contém 12 bolas vermelhas e 18 verdes. Essa amostra será retirada da seguinte maneira: retira a primeira bola, a cor é anotada e a bola é devolvida para a urna, ai então retira a segunda bola, a cor também é anotada e a bola é devolvida para a urna e assim sucessivamente. Qual é a probabilidade de se retirar a sexta bola vermelha na oitava retirada?

Queremos calcular a probabilidade de que a sexta bola vermelha seja retirada na oitava tentativa, logo a oitava bola retirada, obrigatoriamente deve ser vermelha e nas 7 primeiras bolas retiradas, teremos necessariamente 5 bolas vermelhas e e bolas verdes, além disso, a probabilidade de em uma retirada ocorrer bola vermelha é $p=\frac{12}{30}=\frac{2}{5}$ e a probabilidade de ocorrer uma bola verde é $1-p=\frac{3}{5}$.

Usando o teorema das probabilidades binomiais negativas, teremos:

$$
\begin{gathered}
a\left(8,6, \frac{2}{5}\right)=\left(\begin{array}{l}
8-1 \\
6-1
\end{array}\right)\left(\frac{2}{5}\right)^{6} \cdot\left(\frac{3}{5}\right)^{2} \\
=\left(\begin{array}{l}
7 \\
5
\end{array}\right) \frac{64 \cdot 9}{5^{8}} \cong 0,04
\end{gathered}
$$

Exemplo 1.16.5. Um jogador de basquete acerta $80 \%$ dos lances livres que aremessa. Qual é a probabilidade de que ele acerte seu quinto arremesso livre no sétimo arremesso?

A probabilidade de sucesso é $p=\frac{4}{5}$, o número de tentativas $k=7$ e o número de sucessos é $r=5$, então pelo teorema das probabilidades binomiais negativas, teremos

$$
a\left(7,5, \frac{4}{5}\right)=\left(\begin{array}{l}
7-1 \\
5-1
\end{array}\right) \cdot\left(\frac{4}{5}\right)^{5} \cdot\left(\frac{1}{5}\right)^{2} \cong 0,196
$$

\subsection{PROBLEMAS DE PROBABILIDADE COM URNAS}

A mesma estratégia utilizada para resolução de problemas que envolvem urnas pode ser aplicada analogamente para outros problemas por exemplo os termos bolas, cores e urna são termos que podem ser substituidos respectivamente por objetos, tipos de objetos e conjunto de objetos. Cada um dos exemplos a seguir podem ser tratados como problemas com urnas, subconjuntos de pessoas (amostras) são escolhidas dentro de uma comunidade de 
pessoas (urna) e, em uma eleição, estas pessoas votam ou no candidato A ou no candidato B ou no candidato $\mathrm{C}$ (as pessoas são consideradas as bolas da urna com cores distintas em virtude das suas preferências por determinado candidato), também para um lote de produtos manufaturados (a urna) um subconjunto de produtos (a amostra) é selecionada para uma verificação de produtos com defeitos (as bolas de uma cor representam produtos com defeito e bolas da outra cor representam produtos sem defeito). Mãos de

poquer são subconjuntos com cinco cartas retiradas de um baralho de caras (a urna) e cartas de valores diferentes ou de naipes distintos são as bolas de cores diferentes. Os valores ocorridos nos lançamentos sucessivos são amostras de números naturais 1, 2, 3, 4, 5, 6 os quais são considerados urnas com bolas de seis cores diferentes.

Exemplo 1.17.1. O experimento aleatório consiste na retirada em sequência uma a uma de duas bolas de uma urna contendo quatro bolas azuis e duas bolas brancas com a hipótese de que todas as bolas tenham as mesmas chances de serem retiradas. Para o cálculo das probabilidades de

a) ocorrência de duas bolas azuis

b) ocorrência de duas bolas brancas

c) ocorrência de pelo menos uma bola azul

d) ocorrência de duas bolas da mesma cor

Nas seguintes situações:

\section{I) BOLAS DISTINGUÍVEIS}

As quatro bolas azuis são numeradas com os dígitos 1, 2, 3 e 4 e as duas bolas brancas com os dígitos 5 e 6 .

\section{$I_{a}$ ) A retirada das duas bolas é efetuada com reposição na urna da bola retirada na primeira vez.}

O espaço finito equiprovável de probabilidade correspondente ao experimento probabilístico é $\left(\Omega, 2^{\Omega}, P\right)$, cujo espaço amostral é $\Omega$ é dado por

$$
\Omega=\{(i, j): i, j \in\{1,2,3,4,5,6\}\}
$$

e a função de probabilidade $P$ calculada nos subconjuntos unitários $\{(i, j)\} \subset \Omega$ é igual a $P(\{(i, j)\})=\frac{1}{36}$. 
A probabilidade a ser calculada no item a) é a probabilidade do evento $A$ dada por

$$
A=\{(i, j): i, j \in\{1,2,3,4\}\}
$$

que é igual a

$$
P(A)=\frac{|A|}{|\Omega|}=\frac{16}{36}
$$

enquanto que a probabilidade a ser calculada no item b) é a probabilidade do evento $B$ dada por

$$
B=\{(5,5),(5,6),(6,5),(6,6)\}
$$

que é igual a

$$
P(B)=\frac{|B|}{|\Omega|}=\frac{4}{36}
$$

e a probabilidade a ser calculada no item c) é a probabilidade do evento $C$ dada por

$$
\begin{gathered}
C=A \cup\{(1,5),(1,6),(5,1),(6,1),(2,5),(2,6),(5,2),(6,2), \\
(3,5),(3,6),(5,3),(6,3),(4,5),(4,6),(5,4),(6,4)\}
\end{gathered}
$$

que é igual a

$$
P(C)=\frac{|C|}{|\Omega|}=\frac{16+16}{36}=\frac{32}{36}
$$

Como $C=\bar{B}$, a probabilidade do evento $C$ é igual a

$$
P(C)=P(\bar{B})=1-P(B)=1-\frac{4}{36}=\frac{32}{36}
$$

e

$$
P(A \cup B)=P(A)+P(B)=\frac{16}{36}+\frac{4}{36}=\frac{20}{36}
$$

é a resposta à probabilidade requerida no item d).

$I_{b}$ ) A retirada das duas bolas é efetuada sem reposição da primeira bola retirada na urna.

O espaço finito de probabilidade correspondente ao experimento aleatório é $\left(\Omega, 2^{\Omega}, P\right)$, cujo espaço amostral $\Omega$ é dado por

$$
\Omega=\{(i, j): i, j \in\{1,2,3,4,5,6\} i \neq j\}
$$


e a função de probabilidade $P$ calculada no subconjunto unitário $\{(i, j)\} \subset$ $\Omega$ é igual a

$$
P(\{(i, j)\})=\frac{1}{30}
$$

A probabilidade pedida no item a) é a probabilidade do evento $A$

$$
A=\{(i, j): i, j \in\{1,2,3,4\} i \neq j\}
$$

que é igual a

$$
P(A)=\frac{|A|}{|\Omega|}=\frac{4 \cdot 3}{6 \cdot 5}=\frac{12}{30}
$$

enquanto que a probabilidade pedida no item b) é a probabilidade do evento $B$

$$
B=\{(5,6),(6,5)\}
$$

que é igual a

$$
P(B)=\frac{|B|}{|\Omega|}=\frac{2}{30}
$$

e a probabilidade requerida no item c) é a probabilidade do evento $C$

$$
\begin{aligned}
& C=\{\quad(1,2),(1,3),(1,4),(1,5),(1,6), \\
& (2,1),(2,3),(2,4),(2,5),(2,6), \\
& (3,1),(3,2),(3,4),(3,5),(3,6) \text {, } \\
& (4,1),(4,2),(4,3),(4,5),(4,6) \text {, } \\
& (5,1),(5,2),(5,3),(5,4) \text {, } \\
& (6,1),(6,2),(6,3),(6,4)\} \text {. }
\end{aligned}
$$

que é igual a

$$
P(C)=\frac{|C|}{|\Omega|}=\frac{28}{30}
$$

A probabilidade de $C=\bar{B}$ é calculada também por

$$
P(C)=1-P(B)=1-\frac{2}{30}=\frac{28}{30}
$$

$\mathrm{e}$

$$
P(A \cup B)=P(A)+P(B)=\frac{14}{30} .
$$

é a resposta da probabilidade do item d). 


\section{II) BOLAS INDISTINGUÍVEIS}

As quatro bolas azuis são idênticas e as duas bolas brancas são idênticas entre si, enquanto que as seis bolas não são diferenciadas a não ser pela cor.

\section{$I I_{a}$ ) A retirada das duas bolas é efetuada com reposição na urna da bola retirada na primeira vez.}

O espaço finito de probabilidade correspondente ao experimento aleatório é $\left(\Omega, 2^{\Omega}, P\right)$, cujo espaço amostral $\Omega$ é dado por

$$
\Omega=\{(0,0),(0,1),(1,0),(1,1)\}
$$

em que o dígito 0 indica a retirada de bola azul e o dígito 1 indica a retirada de bola branca: o par $(1,0)$ indica que a primeira bola retirada é branca e a segunda bola retirada é azul. A função probabilidade $P$ é definida nos subconjuntos unitários de $\Omega$ por

$$
\begin{aligned}
& P(\{(0,0)\})=\frac{4}{6} \cdot \frac{4}{6} \\
& P(\{(0,1)\})=\frac{4}{6} \cdot \frac{2}{6} \\
& P(\{(1,0)\})=\frac{2}{6} \cdot \frac{4}{6} \\
& P(\{(1,1)\})=\frac{2}{6} \cdot \frac{2}{6}
\end{aligned}
$$

A probabilidade do item a) é a probabilidade do evento $A$ dado por

$$
A=\{(0,0)\}
$$

igual a

$$
P(A)=\frac{4}{6} \cdot \frac{4}{6}
$$

enquanto que a probabilidade do item b) é igual a probabilidade do evento $B$ dada por

$$
B=\{(1,1)\}
$$

que é igual a

$$
P(B)=\frac{2}{6} \cdot \frac{2}{6}
$$

e que a probabilidade do caso c) é a probabilidade do evento $C$

$$
C=\{(0,0),(01),(1,0)\}
$$


igual a

$$
\begin{aligned}
P(C) & =P(\{(0,0)\})+P(\{(0,1)\})+P(\{(1,0)\}) \\
& =\frac{4}{6} \cdot \frac{4}{6}+\frac{4}{6} \cdot \frac{2}{6}+\frac{2}{6} \cdot \frac{4}{6}
\end{aligned}
$$

como $C=\bar{B}$, a probabilidade do evento $C$ pode ser calculada por

$$
P(C)=1-P(B)=1-\frac{2}{6} \cdot \frac{2}{6}
$$

$\mathrm{e}$

$$
P(A \cup B)=P(A)+P(B)=\frac{4}{6} \cdot \frac{4}{6}+\frac{2}{6} \cdot \frac{2}{6}
$$

é a resposta do caso d).

$\left.I I_{b}\right)$ A retirada das duas bolas é efetuada sem reposição na urna, ou seja, a bola retirada na primeira vez não volta para a urna.

O espaço finito de probabilidade corresponde ao experimento aleatório é $\left(\Omega, 2^{\Omega}, P\right)$ cujo espaço amostral $\Omega$ é dado por

$$
\Omega=\{(0,0),(0,1),(1,0),(1,1)\}
$$

e a função de probabilidade $P$ é definida nos subconjuntos unitários de $\Omega$ por

$$
\begin{aligned}
& P(\{(0,0)\})=\frac{4}{6} \cdot \frac{3}{5} \\
& P(\{(0,1)\})=\frac{4}{6} \cdot \frac{2}{5} \\
& P(\{(1,0)\})=\frac{2}{6} \cdot \frac{4}{5} \\
& P(\{(1,1)\})=\frac{2}{6} \cdot \frac{1}{5}
\end{aligned}
$$

A probabilidade do caso a) é a probabilidade do evento $A$

$$
A=\{(0,0)\}
$$

igual a

$$
P(A)=\frac{4}{6} \cdot \frac{3}{5}
$$

enquanto que a probabilidade do caso b) é a probabilidade do evento $B$

$$
B=\{(1,1)\}
$$


igual a

$$
P(B)=\frac{2}{6} \cdot \frac{1}{5}
$$

e a probabilidade do caso c) é a probabilidade do evento $C$

$$
C=\{(0,0),(0,1),(1,0)\}
$$

igual a

$$
\begin{gathered}
P(C)=P(\{(0,0)\})+P(\{(0,1)\})+P(\{(1,0)\}) \\
=\frac{4}{6} \cdot \frac{3}{5}+\frac{4}{6} \cdot \frac{2}{5}+\frac{2}{6} \cdot \frac{4}{5}
\end{gathered}
$$

cujo valor, também pode ser calculado por

$$
P(C)=1-P(B)=1-\frac{2}{6} \cdot \frac{1}{5}
$$

pois $C=\bar{B}$ e

$$
P(A \cup B)=P(A)+P(B)=\frac{4}{6} \cdot \frac{3}{5}+\frac{2}{6} \cdot \frac{1}{5}=\frac{14}{30}
$$

é a resposta do caso d).

\subsection{UM PROBLEMA COM N URNAS}

O experimento aleatório consiste na escolha de uma urna entre $N$ urnas numeradas com os dígitos $1,2, \ldots, N$ : para cada $j \in\{1,2, \ldots, N\}$, a urna $j$ tem $a_{j}$ bolas azuis e $b_{j}$ bolas brancas e na retirada de uma bola da urna escolhida com a hipótese de que todas as bolas não são diferenciadas a não ser pela cor.

A probabilidade a ser calculada é a probabilidade de ocorrência da retirada de uma bola branca. Com a hipótese de que todas as urnas tem a mesma chance de serem escolhidas e de que todas as bolas tem a mesma chance de serem extraídas. O espaço finito de probabilidade correspondente é $\left(\Omega, 2^{\Omega}, P\right)$ cujo espaço amostral $\Omega$ é dado por

$$
\Omega=\{1,2, \ldots, N\} \times\{1,0\}
$$

em que o dígito 0 indica bola azul e o dígito 1 indica bola branca e a função de probabilidade $P$ é definida nos subconjuntos unitários de $\Omega$ por

$$
P(\{(j, 0)\})=\frac{1}{N} \cdot \frac{a_{j}}{a_{j}+b_{j}}, j=1,2, \ldots, N
$$




$$
P(\{(j, 1)\})=\frac{1}{N} \cdot \frac{b_{j}}{a_{j}+b_{j}}, j=1,2, \ldots, N
$$

Como

$$
\Omega=\bigcup_{j=1}^{N} H_{j}
$$

em que para cada $j=1,2, \ldots, N, H_{j}=\{(j, 0),(j, 1)\}$, cuja probabilidade $P\left(H_{j}\right)=\frac{1}{N}$ o teorema total de Bayes garante que

$$
\begin{aligned}
P(A) & =P\left(A \cap \bigcup_{j=1}^{N} H_{j}\right)=P\left(\bigcup_{j=1}^{N}\left(A \cap H_{j}\right)\right)=P\left(A \cap H_{1}\right)+\ldots+P\left(A \cap H_{N}\right) \\
& =P\left(A \mid H_{1}\right) P\left(H_{1}\right)+P\left(A \mid H_{2}\right) P\left(H_{2}\right)+\ldots+P\left(A \mid H_{N}\right) P\left(H_{N}\right) \\
& =\frac{1}{N} \cdot\left[\frac{b_{1}}{a_{1}+b_{1}}+\frac{b_{2}}{a_{2}+b_{2}}+\ldots+\frac{b_{N}}{a_{N}+b_{N}}\right]
\end{aligned}
$$

Exemplo 1.18.1. Uma urna $A$ contém 3 bolas vermelhas e 3 bolas pretas, enquanto a urna $B$ contém 4 bolas vermelhas e 6 bolas pretas. Uma urna é escolhida ao acaso e posteriormente uma bola é retirada dessa urna, a probabilidade de que a bola retirada seja da cor vermelha é dada por

$$
\frac{1}{2} \cdot\left[\frac{3}{3+3}+\frac{4}{4+6}\right]=\frac{9}{20}
$$

Com a hipótese de que as duas urnas tem a mesma chance de serem escolhidas e com a hipótese de que todas as bolas tem a mesma chance de serem extraídas.

\subsubsection{AMOSTRAS NÃO ORDENADAS}

O experimento aleatório consiste na retirada de uma amostra não ordenada de duas bolas de uma urna contendo quatro bolas azuis e duas bolas brancas: as bolas azuis são numeradas com os dígitos 1,2,3,4 e as bolas brancas são numeradas com os dígitos 5,6 com a hipótese de que todas as bolas tem a mesma chance de fazer parte da amostra não ordenada. O objetivo é o cálculo das probabilidades de

a) ocorrência de duas bolas azuis;

b) ocorrência de duas bolas brancas;

c) ocorrência de pelo menos uma bola azul; 
d) ocorrência de duas bolas com a mesma cor.

O espaço de probabilidade correspondente é o espaço finito de probabilidade $\left(\Omega, 2^{\Omega}, P\right)$ cujo espaço amostral $\Omega$ é o conjunto de todos os $\left(\begin{array}{l}6 \\ 2\end{array}\right)$ subconjuntos de $\Omega$ com dois elementos e a função de probabilidade $P$ calculada em $\{i, j\}$ com $i, j \in\{1,2,3,4,5,6\}$ com $i \neq j$ é definida por

$$
P(\{i, j\})=\frac{1}{\left(\begin{array}{l}
6 \\
2
\end{array}\right)}=\frac{1}{15}
$$

A probabilidade do caso a) é a probabilidade do evento $A$ constituído por todos os subconjuntos de dois elementos do conjunto $\{1,2,3,4\}$

$$
P(A)=\frac{|A|}{|\Omega|}=\frac{\left(\begin{array}{l}
4 \\
2
\end{array}\right)}{\left(\begin{array}{l}
6 \\
2
\end{array}\right)}=\frac{6}{15}
$$

e a probabilidade do evento $B=\{5,6\}$

$$
P(B)=\frac{|B|}{|\Omega|}=\frac{\left(\begin{array}{l}
2 \\
2
\end{array}\right)}{\left(\begin{array}{l}
6 \\
2
\end{array}\right)}=\frac{1}{15}
$$

A probabilidade do caso c) é a probabilidade do evento $C=\bar{B}$ e

$$
P(C)=P(\bar{B})=1-P(B)=1-\frac{1}{15}=\frac{14}{15}
$$

$\mathrm{e}$

$$
P(A \cup B)=P(A)+P(B)=\frac{6}{15}+\frac{1}{15}=\frac{7}{15}
$$

é a resposta do caso d).

O experimento aleatório consiste na retirada de uma amostra não ordenada de duas bolas de uma urna contendo quatro bolas azuis e duas bolas brancas em que as bolas não são diferenciadas a não ser pela cor com a hipótese de que todas as amostras não ordenadas são equiprováveis.

O espaço de probabilidade correspondente é o espaço finito de probabilidade $\left(\Omega, 2^{\Omega}, P\right)$ cujo espaço amostral $\Omega$ é composto de todas as $\left(\begin{array}{l}3 \\ 2\end{array}\right)=3$ soluções inteiras não negativas $(x, y)$ da equação

$$
x+y=2, \operatorname{com} x=0,1,2 \text { e } y=0,1,2
$$

em que $x$ indica o número de de bolas azuis e $y$ indica o número de bolas brancas da amostra não ordenada. Assim, as probabilidades dos casos a), b), c), d) são respectivamente iguais a 


$$
\begin{aligned}
& P(A)=\frac{|A|}{|\Omega|}=\frac{1}{3} \text { em que } A=\{(2,0)\} \\
& P(B)=\frac{|B|}{|\Omega|}=\frac{1}{3} \text { em que } A=\{(0,2)\}
\end{aligned}
$$

Como $C=\bar{B}$,

$$
\begin{gathered}
P(C)=P(\bar{B})=1-P(B)=1-\frac{1}{3}=\frac{2}{3} \\
P(A \cup B)=P(A)+P(B)=\frac{2}{3}
\end{gathered}
$$

\subsection{CÁLCULO DE PROBABILIDADE DE AMOS- TRAS ORDENADAS}

\subsubsection{CASO A - Cálculo de probabilidade de amostras or- denadas com reposição de elementos de um conjunto não vazio}

Seja $U$ um conjunto não vazio com $N$ elementos e seja $V$ um subconjunto não vazio de $U$ com $a$ elementos.

O experimento aleatório consiste na escolha sequencial de $k$ elementos de $U$ com $k \leq N$, isto é, um a um os elementos de $U$ são selecionados com a hipótese de que todos elementos de $U$ tem a mesma chance de serem selecionados e com a hipótese de que um elemento de $U$ pode não ocorrer ou pode ocorrer uma ou mais vezes na sequência. Então, a probabilidade de que o primeiro elemento selecionado pertença ao subconjunto $V$ de $U$ é igual a

$$
\frac{a}{N}
$$

e para cada $j=1,2, \ldots, k$ a probabilidade de que o elemento número $j$ pertença ao subconjuto $V$ de $U$ também é

$$
\frac{a}{N}
$$

Demonstração. O modelo probabilístico $\left(\Omega, 2^{\Omega}, P\right)$ tem como espaço amostral $\Omega$, que é o conjunto de todas as $N^{k}$ sequências finitas formadas por $k$ elementos de $\Omega$, isto é, $\Omega=\left\{\left(u_{1}, u_{2}, \ldots, u_{k}\right): u_{1}, u_{2}, \ldots, u_{k} \in \Omega\right\}$ e o evento 
$A_{1} \subset \Omega$ constituído por todas as sequências em que o primeiro elemento $u_{1} \in V$ intuitivamente tem por probabilidade

$$
P\left(A_{1}\right)=\frac{a}{a+n-a}=\frac{a}{n}
$$

mas para cada $j=1,2, \ldots, k$, a probabilidade do evento $A_{j}$ constituído por todas as $a n^{k-1}$ sequências de $\Omega$ em que o j-ésimo elemento $u_{j}$ é um elemento de $V$, é igual a

$$
P\left(A_{j}\right)=\frac{\left|A_{j}\right|}{|\Omega|}=\frac{a n^{k-1}}{n^{k}}=\frac{a}{n}
$$

\subsubsection{CASO B - Cálculo de probabilidade de amostras or- denadas sem reposição de elementos de um conjunto não vazio}

Seja $U$ um conjunto não vazio com $N$ elementos e seja $V$ um subconjunto não vazio de $U$ com $a$ elementos.

O experimento aleatório consiste na escolha sequencial um a um de $k$ elementos de $U$ em que $k \leq N$, isto é um a um os elementos de $U$ são escolhidos de forma aleatória com a hipótese de que todas os elementos de $U$ tem a mesma chance de serem selecionados e com a hipótese de que um elemento ocorre no máximo uma vez na sequência . Então, a probabilidade de que o segundo elemento da sequência pertença ao subconjunto $V$ de $U$ é igua a

$$
\frac{a}{N}
$$

Para cada $j=1,2, \ldots, k$, a probabilidade de que o elemento de número $j$ da sequência pertence ao subconjunto $V$ de $U$ também é

$$
\frac{a}{N}
$$

Demonstração. para o caso $j=2$

O modelo probabilistico $\left(\Omega, 2^{\Omega}, P\right)$ tem por espaço amostral $\Omega$ o conjunto de todos as $N \cdot(N-1) \cdot(N-2) \cdot \ldots \cdot(N-k+1)$ sequências de $k$ elementos de $\Omega$ contituídas pelos elementos dois a dois distintos entre si e o evento $A_{2}$ é contituído pelas sequências de $\Omega$, em que o segundo elemento é um elemento de $V$, tem por probabilidade

$$
P\left(A_{2}\right)=\frac{a}{N}
$$


desde que $A_{2}=\left(A_{1} \cap A_{2}\right) \cup\left(\overline{A_{1}} \cap A_{2}\right)$, em que $A_{1}$ é o conjunto das sequências de $\Omega$ cujo primeiro elemento pertence a $V$, e

$$
P\left(A_{2}\right)=P\left(A_{1} \cap A_{2}\right)+\left(\overline{A_{1}} \cap A_{2}\right)
$$

pelo terceiro postulado de Kolmogorov, mas

$$
\begin{aligned}
P\left(A_{1} \cap A_{2}\right) & =\frac{\left|A_{1} \cap A_{2}\right|}{|\Omega|} \\
& =\frac{a \cdot(a-1) \cdot(N-2) \cdot \ldots \cdot(N-2+k+3)}{n \cdot(N-1) \cdot \ldots \cdot(N-k+1)} \\
& =\frac{a \cdot(a-1)}{N \cdot(N-1)}
\end{aligned}
$$

enquanto que

$$
P\left(\overline{A_{1}} \cap A_{2}\right)=\frac{\left|\overline{A_{1}} \cap A_{2}\right|}{|\Omega|}=\frac{(N-a) \cdot a}{N \cdot(N-1)}
$$

o que mostra que

$$
P\left(A_{2}\right)=\frac{a \cdot(a-1)+a \cdot(N-a)}{N \cdot(N-1)}=\frac{a}{n}
$$

\subsubsection{CASO C}

Seja $U=\left\{u_{1}, u_{2}, \ldots, u_{N}\right\}$ um conjunto não vazio com $N$ elementos.

Dados $n, k \in\{1,2,3, \ldots\}$ com $k \leq n$, a probabilidade de ocorrência de uma sequência ordenada com $n$ elementos de $U$ escolhidos de forma aleatória, em que cada elemento de $U$ pode aparecer zero, uma ou mais de uma vez na sequência e em que o elemento $u_{1} \in U$ ocorre $k$ vezes na sequência, é igual a

$$
\left(\begin{array}{l}
n \\
k
\end{array}\right) \cdot \frac{(N-1)^{n-k}}{N^{n}}
$$

com a hipótese de que todos os elementos de $U$ tem a mesma chance de serem escolhidos.

Demonstração. O experimento aleatório consiste na retirada em sequência uma a uma de $n$ bolas de uma urna com reposição na urna da bola tirada a cada passo. A urna contém $N$ bolas numeradas com os dígitos $1,2, \ldots, N$ em que a chance de retirada de uma bola é a mesma para todas as bolas. 
O espaço de probabilidade equiprovável finito correspondente ao experimento aleatório é $\left(\Omega, 2^{\Omega}, P\right)$, cujo espaço amostral $\Omega$ é o um conjunto de todas as $n^{k}$ sequências de elementos de $U$.

A probabilidade do elemento $u_{1}$ ocorrer $k$ vezes na amostra ordenada de $n$ elementos do conjunto $U$ de $N$ elementos em que $k \leq n$ é igual a

$$
\frac{\left(\begin{array}{l}
n \\
k
\end{array}\right)(N-1)^{n-k}}{N^{n}}, k=0,1,2, \ldots, n
$$

\subsubsection{CASO D}

Seja $U=\left\{u_{1}, u_{2}, \ldots, u_{N}\right\}$ um conjunto não vazio com $N$ elementos.

Dados $n, k_{1}, k_{2}, \ldots, k_{N} \in\{1,2,3, \ldots\}$ com $n=k_{1}+k_{2}+\ldots+k_{N}$ a probabilidade de ocorrência de uma sequência ordenada de $n$ elementos de $U$ escolhidos de forma aleatória em que cada elemento de $U$ pode aparecer zero, uma ou mais de uma vez na sequência e em que o elemento $u_{1}$ ocorre $k_{1}$ vezes, o elemento $u_{2}$ ocorre $k_{2}$ vezes e assim por diante, o elemento $u_{N}$ ocorre $k_{N}$ vezes na sequência, é igual a

$$
\left(\frac{n !}{k_{1} ! \cdot k_{2} ! \cdot \ldots \cdot k_{N} !}\right) \cdot \frac{1}{N^{n}}, \operatorname{com} n=k_{1}+k_{2}+\ldots+k_{N}
$$

com a hipótese de que todos os elementos de $U$ tem a mesma chance de serem escolhidos.

\subsubsection{CASO E}

Seja $U=\left\{u_{1}, u_{2}, \ldots, u_{N}\right\}$ um conjunto não vazio com $N$ elementos.

Dados $n, k \in\{1,2,3, \ldots\}$ com $k \leq n$, a probabilidade de ocorrência de uma sequência ordenada com $n$ elementos de $U$ escolhidos de forma aleatória, em que cada elemento de $U$ pode aparecer zero, uma ou mais de uma vez na sequência e em que os primeiros $k$ elementos $u_{1}, u_{2}, \ldots, u_{k}$ de $U$ obrigatotiamente ocorrem na sequência, é igual a

$$
\frac{1}{N^{n}} \sum_{j=0}^{k}(-1)^{j}\left(\begin{array}{l}
k \\
j
\end{array}\right)(N-j)^{n}
$$

com a hipótese de que todos os elementos de $U$ tem a mesma chance de serem escolhidos. 


\subsubsection{CASO F}

Seja $U=\left\{u_{1}, u_{2}, \ldots, u_{N}\right\}$ um conjunto não vazio com $N$ elementos.

Dados $n, k, m \in\{1,2,3, \ldots\}$ com $0 \leq m \leq k \leq n$, a probabilidade de ocorrência de uma sequência ordenada com $n$ elementos de $U$ escolhidos de forma aleatória, em que cada elemento de $U$ pode aparecer zero, uma ou mais de uma vez na sequência e em que o número exato $m$ dos $k$ elementos $u_{1}, u_{2}, \ldots, u_{k}$ aparece na sequência, é igual a

$$
\begin{gathered}
\frac{1}{N^{n}} \sum_{j=m}^{N}(-1)^{j-m}\left(\begin{array}{c}
k \\
j
\end{array}\right)\left(\begin{array}{c}
j \\
m
\end{array}\right)(N-j)^{n} \\
=\frac{1}{N^{n}}\left(\begin{array}{c}
N \\
m
\end{array}\right) \sum_{j=0}^{N-m}(-1)^{j}\left(\begin{array}{c}
k-m \\
j
\end{array}\right)(N-j-m)^{n}
\end{gathered}
$$

com a hipótese de que todos os elementos de $U$ tem a mesma chance de serem escolhidos.

\subsection{CÁLCULO DE PROBABILIDADE DE AMOS- TRAS NÃO ORDENADAS}

\subsubsection{CASO A}

Seja $U=\left\{u_{1}, u_{2}, \ldots, u_{N}\right\}$ um conjunto não vazio com $N$ elementos.

Dados $n \in\{1,2,3, \ldots, N\}$, a probabilidade de ocorrência de um subconjunto com $n$ elementos de $U$ escolhidos de forma aleatória, em que o elemento $u_{1}$ de $U$

i) não ocorre no subconjunto é igual a

$$
\frac{\left(\begin{array}{c}
N-1 \\
n
\end{array}\right)}{\left(\begin{array}{c}
N \\
n
\end{array}\right)}
$$

ii) ocorre no subconjunto é igual a

$$
\frac{\left(\begin{array}{l}
N-1 \\
n-1
\end{array}\right)}{\left(\begin{array}{l}
N \\
n
\end{array}\right)}
$$

com a hipótese de que todos os elementos de $U$ tem a mesma chance de serem escolhidos. 


\subsubsection{CASO B}

Seja $U=\left\{u_{1}, u_{2}, \ldots, u_{N}\right\}$ um conjunto não vazio com $N$ elementos.

Dados $n \in\{1,2,3, \ldots, N\}$ e $k_{1}, k_{2}, \ldots, k_{N} \in\{0,1\}$, a probabilidade de ocorrência de um subconjunto com $n$ elementos de $U$ escolhidos de forma aleatória, em que o elemento $u_{1}$ ocorre $k_{1}$ vezes, o elemento $u_{2}$ ocorre $k_{2}$ vezes e assim por diante, o elemento $u_{N}$ ocorre $k_{N}$ vezes, é igual a

$$
\frac{1}{\left(\begin{array}{l}
N \\
n
\end{array}\right)}
$$

com a hipótese de que todos os elementos de $U$ tem a mesma chance de serem escolhidos.

\subsubsection{CASO C}

Seja $U=\left\{u_{1}, u_{2}, \ldots, u_{N}\right\}$ um conjunto não vazio com $N$ elementos.

Dados $n, k, m \in\{1,2,3, \ldots, N\}$ com $m \leq k \leq n$, a probabilidade de ocorrência de um subconjunto com $n$ elementos de $U$ escolhidos de forma aleatória, em que cada um dos elementos $u_{1}, u_{2}, \ldots, u_{m}$ pertence ao subconjunto é igual a

$$
\begin{gathered}
\frac{1}{(N)_{n}} \sum_{j=0}^{m}(-1)^{j}\left(\begin{array}{c}
m \\
j
\end{array}\right)(N-j)_{n}= \\
=\frac{\left(\begin{array}{l}
N-m \\
n-m
\end{array}\right)}{\left(\begin{array}{l}
N \\
n
\end{array}\right)}
\end{gathered}
$$

com a hipótese de que todos os elementos de $U$ tem a mesma chance de serem escolhidos.

\subsubsection{CASO D}

Seja $U=\left\{u_{1}, u_{2}, \ldots, u_{N}\right\}$ um conjunto não vazio com $N$ elementos.

Dados $n, k, m \in\{1,2,3, \ldots, N\}$ com $m \leq k \leq n$, a probabilidade de ocorrência de um subconjunto com $n$ elementos de $U$ escolhidos de forma aleatória, em que o número exato $m$ de elementos $u_{1}, u_{2}, \ldots, u_{k}$ ocorra em um subconjunto, é igual a

$$
\frac{1}{(n)_{n}} \sum_{j=m}^{N}(-1)^{j}\left(\begin{array}{c}
k \\
j
\end{array}\right)\left(\begin{array}{c}
j \\
m
\end{array}\right)(N-j)_{n}=
$$




$$
=\frac{\left(\begin{array}{c}
k \\
m
\end{array}\right)\left(\begin{array}{c}
N-k \\
n-k+m
\end{array}\right)}{\left(\begin{array}{c}
N \\
n
\end{array}\right)}
$$

com a hipótese de que todos os elementos de $U$ tem a mesma chance de serem escolhidos.

\subsection{PROBLEMAS DE OCUPAÇÃO EM URNAS COM BOLAS IDÊNTICAS}

\subsubsection{CASO A}

A probabilidade da urna número um conter exatas $k$ bolas idênticas na distribuição aleatória de $n$ bolas idênticas em $N$ urnas numeradas com os dígitos $1,2, \ldots, N$ é igual a

$$
\frac{\left(\begin{array}{c}
N+n-k-2 \\
n-k
\end{array}\right)}{\left(\begin{array}{c}
N+n-1 \\
n
\end{array}\right)}
$$

desde que $n, N \in\{1,2,3, \ldots\}$ e $k \in\{0,1,2, \ldots, n\}$ com a hipótese de que a chance de uma urna conter uma bola é a mesma para todas as urnas e todas as bolas.

\subsubsection{CASO B}

Dados $k_{1}, k_{2}, \ldots, k_{N} \in\{0,1,2, \ldots, n\}$ com $\sum_{j=1}^{N} k_{j}=n$ a probabilidade de que para cada $j=1,2, \ldots, N$ a urna número $j$ contém $k_{j}$ bolas idênticas em $N$ urnas numeradas com os dígitos $1,2, \ldots, N$ é igual a

$$
\frac{1}{\left(\begin{array}{c}
N+n-1 \\
n
\end{array}\right)}
$$

desde que $n, N \in\{1,2,3, \ldots\}$ com a hipótese de que a chance de uma urna conter uma bola é a mesma para todas as urnas e todas as bolas.

\subsubsection{CASO C}

A probabilidade de que as primeiras $M$ urnas numeradas de 1 a $M$ não fiquem vazias na distribuição aleatória de $n$ bolas idênticas em $N$ urnas numeradas com os dígitos $1,2, \ldots, M, \ldots, N$ com $M \in\{1,2, \ldots, N\}$ e $n \geq M$ é igual a

$$
\frac{\left(\begin{array}{c}
N-M+n-1 \\
n-M
\end{array}\right)}{\left(\begin{array}{c}
N+n-1 \\
n
\end{array}\right)}
$$


desde que $n, N \in\{1,2,3, \ldots\}$ e $M \in\{0,1,2, \ldots, N\}$ com a hipótese de que a chance de uma urna conter uma bola é a mesma para todas as urnas e todas as bolas.

Demonstração. O modelo probabilístico é o espaço finito equiprovável de probabilidade $\left(\Omega, 2^{\Omega}, P\right)$, cujo espaço amostral $\Omega$ é o conjunto de todas as sequências ordenadas de $N$ elementos $\left(z_{1}, z_{2}, \ldots, z_{N}\right)$ em que, para cada $j=$ $1,2, \ldots, N, z_{j}$ indica o número de bolas contida na urna $j$ e o número de elementos de $|\Omega|$ do espaço amostral $\Omega$ é o número de soluções inteiras não negativas da equação

$$
z_{1}+z_{2}+\ldots+z_{N}=n
$$

com $z_{1}, z_{2}, \ldots, z_{N} \in\{0,1,2, \ldots, n\}$ que é igual a

$$
|\Omega|=\left(\begin{array}{c}
N+n-1 \\
n
\end{array}\right)
$$

O evento $A$ é o subconjunto de $\Omega$ constituído pela totalidade das sequências ordenadas de $N$ elementos

$$
\left(z_{1}, z_{2}, \ldots, z_{M}, \ldots, z_{N}\right)
$$

em que $z_{1} \geq 1, z_{2} \geq 1, \ldots, z_{M} \geq 1$ e o número de elementos de $|A|$ do evento $A$ é o número de soluções inteiras não negativas da equação

$$
\begin{gathered}
z_{1}+z_{2}+\ldots+z_{M}+\ldots+z_{N}=n \\
z_{1}, z_{2}, \ldots, z_{M} \in\{1,2, \ldots, n\} \\
z_{M+1}, z_{M+2}, \ldots, z_{N} \in\{0,1,2, \ldots, n\}
\end{gathered}
$$

que é igual a

$$
|A|=\left(\begin{array}{c}
N-M+n-1 \\
n-M
\end{array}\right)
$$

A probabilidade a ser calculada é

$$
P(A)=\frac{|A|}{|\Omega|}=\frac{\left(\begin{array}{c}
N-M+n-1 \\
n-M
\end{array}\right)}{\left(\begin{array}{c}
N+n-1 \\
n
\end{array}\right)}
$$

com a hipótese adicional de que $n \geq M$. 


\subsubsection{CASO D}

A probabilidade de que exatas $P$ das primeiras $M$ urnas numeradas de 1 a $M$ permaneçam vazias na distribuição aleatória de $n$ bolas idênticas em $N$ urnas numeradas com os dígitos $1,2, \ldots, N$ é igual a

$$
\frac{\left(\begin{array}{c}
M \\
P
\end{array}\right)\left(\begin{array}{c}
N-M+n-1 \\
N-P-1
\end{array}\right)}{\left(\begin{array}{c}
N+n-1 \\
n
\end{array}\right)}
$$

desde que $n, N \in\{1,2,3, \ldots\}, P \in\{0,1,2, \ldots, M\}$ e $M \in\{0,1,2, \ldots, N\}$ com a hipótese de uma urna conter uma bola é a mesma para todas as urnas e todas as bolas.

\subsection{PROBLEMAS DE OCUPAÇÃO EM URNAS COM BOLAS IDÊNTICAS OU COM BOLAS NUMERADAS}

\subsubsection{CASO A}

A probabilidade da urna número um conter uma única bola na distribuição aleatória de $n$ bolas idênticas ou na distribuição aleatória de $n$ bolas numeradas em $N$ urnas numeradas com os dígitos $1,2, \ldots, N$ é igual a

$$
\frac{\left(\begin{array}{l}
N-1 \\
n-1
\end{array}\right)}{\left(\begin{array}{l}
N \\
n
\end{array}\right)}
$$

desde que $n, N \in\{1,2,3, \ldots\}$ e desde que a distribuição aleatória está sujeita ao princípio da exclusão: cada urna contém no máximo uma bola, com a hipótese de que a chance de uma urna conter uma bola é a mesma para todas as urnas e todas as bolas.

\subsubsection{CASO B}

A probabilidade da urna número um ficar vazia na distribuição aleatória de $n$ bolas idênticas ou na distribuição aleatória de $n$ bolas numeradas em $N$ urnas numeradas com os dígitos $1,2, \ldots, N$ é igual a

$$
\frac{\left(\begin{array}{c}
N-1 \\
n
\end{array}\right)}{\left(\begin{array}{c}
N \\
n
\end{array}\right)}
$$

desde que $n, N \in\{1,2,3, \ldots\}$ e desde que a distribuição aleatória está sujeita ao princípio da exclusão: cada urna contém no máximo uma bola, com a 
hipótese de que a chance de uma urna conter uma bola é a mesma para todas as urnas e todas as bolas.

\subsubsection{CASO C}

A probabilidade de que para cada $j=1,2, \ldots, N$ a urna número $j$ contem $k_{j}$ bolas em que $k_{1}+k_{2}+\ldots+k_{N}=n$ e $k_{1}, k_{2}, \ldots, k_{N} \in\{0,1\}$ na distribuição aleatória de $n$ bolas idênticas ou na distribuição aleatória de $n$ bolas numeradas em $N$ urnas numeradas com os dígitos $1,2, \ldots, N$ é igual a

$$
\frac{1}{\left(\begin{array}{l}
N \\
n
\end{array}\right)}
$$

desde que $n, N \in\{1,2,3, \ldots\}$ com a hipótese de que a chance de uma urna conter uma bola é a mesma para todas as urnas e todas as bolas.

\subsubsection{CASO D}

A probabilidade de que as primeiras $M$ urnas das $N$ urnas numeradas com $n$ dígitos $1,2, \ldots, N$ contenham uma única bola na distribuição aleatória de $n$ bolas idênticas ou na distribuição aleatória de $n$ bolas numeradas em $N$ urnas numeradas com os dígitos $1,2, \ldots, N$ é igual a

$$
\begin{gathered}
\frac{1}{(N)_{n}} \sum_{j=0}^{M}(-1)^{j}\left(\begin{array}{c}
M \\
j
\end{array}\right)(N-j)_{n}= \\
=\frac{\left(\begin{array}{c}
N-M \\
n-N
\end{array}\right)}{\left(\begin{array}{l}
N \\
n
\end{array}\right)}
\end{gathered}
$$

desde que $n, N \in\{1,2,3, \ldots\}$ e $M \in\{0,1,2, \ldots, N\}$ e desde que a distribuição aleatória está sujeita ao princípio de exclusão: cada urna contém no máximo uma bola, com a hipótese de que a chance de uma urna conter uma bola é a mesma para todas as urnas e todas as bolas.

\subsubsection{CASO E}

A probabilidade de que exatas $P$ das primeiras $M$ urnas numeradas entre as $N$ urnas numeradas com os dígitos $1,2, \ldots, N$ permanecem vazias na distribuição aleatória de $n$ bolas idênticas ou na distribuição aleatória de $n$ bolas numeradas em $N$ urnas numeradas é igual a

$$
\frac{1}{(N)_{n}} \sum_{j=P}^{M}(-1)^{j}\left(\begin{array}{c}
M \\
j
\end{array}\right)\left(\begin{array}{c}
j \\
P
\end{array}\right)(N-j)_{n}=
$$




$$
=\frac{\left(\begin{array}{c}
M \\
P
\end{array}\right)\left(\begin{array}{c}
N-M \\
n-M+P
\end{array}\right)}{\left(\begin{array}{l}
N \\
n
\end{array}\right)}
$$

desde que $n, N \in\{1,2,3, \ldots\}, P \in\{0,1,2, \ldots, M\}$ e $M \in\{0,1,2, \ldots, N\}$ e desde que a distribuição aleatória está sujeita ao princípio de exclusão:(com a hipótese de que a chance de uma urna conter uma bola é a mesma para todas as urnas e todas as bolas).

\subsection{PROBLEMAS DE OCUPAÇÃO EM URNAS COM BOLAS NUMERADAS}

\subsubsection{CASO A}

Dados $n, N \in\{1,2,3, \ldots\}$ e $k \in\{0,1,2, \ldots, n\}$ a probabilidade de ocorrência de uma distribuição aleatória de $n$ bolas numeradas de 1 a $n$ em $N$ urnas numeradas de 1 a $N$ em que a urna número um contém $k$ bolas é igual a

$$
\frac{\left(\begin{array}{l}
n \\
k
\end{array}\right)(N-1)^{n-k}}{N^{n}}
$$

com a hipótese de que a chance de uma urna conter uma bola é a mesma para todas as urnas e todas as bolas.

\subsubsection{CASO B}

Dados $n, N \in\{1,2,3, \ldots\}$ e $k_{1}, k_{2}, \ldots, k_{N} \in\{0,1,2, \ldots, n\}$ a probabilidade de ocorrência de uma distribuição aleatória de $n$ bolas numeradas de 1 a $n$ em $N$ urnas numeradas de 1 a $N$ em que a urna número um contém $k_{1}$ bolas, a urna número dois comtém $k_{2}$ bolas e assim por diante a urna número $N$ contém $k_{N}$ bolas é igual a

$$
\left(\frac{1}{N^{n}}\right) \cdot\left(\frac{n !}{k_{1} ! \cdot k_{2} ! \cdot \ldots \cdot k_{N} !}\right)
$$

com a hipótese de que a chance de uma urna conter uma bola é a mesma para todas as urnas e todas as bolas.

\subsubsection{CASO C}

Dados $n, N \in\{1,2,3, \ldots\}$ e $M \in\{0,1,2, \ldots, N\}$ a probabilidade de ocorrência de uma distribuição aleatória de $n$ bolas numeradas de 1 a $n$ em $N$ 
urnas numeradas de 1 a $N$ em que as primeiras $M$ urnas numeradas de 1 a $M$ contém no mímino uma bola é igual a

$$
\frac{1}{N^{n}} \sum_{j=0}^{M}(-1)^{j}\left(\begin{array}{c}
M \\
j
\end{array}\right)(N-j)^{n}
$$

com a hipótese de que a chance de uma urna conter uma bola é a mesma para todas as urnas e todas as bolas.

\subsubsection{CASO D}

Dados $n, N \in\{1,2,3, \ldots\}, M \in\{0,1,2, \ldots, N\}$ e $m \in\{0,1,2, \ldots, M\}$ a probabilidade de ocorrência de uma distribuição aleatória de $n$ bolas numeradas de 1 a $n$ em $N$ urnas numeradas de 1 a $N$ em que o número exato $m$ das $M$ primeiras urnas numeradas de 1 a $M$ ficam vazias é igual a

$$
\frac{1}{N^{n}}\left(\begin{array}{l}
M \\
m
\end{array}\right) \sum_{j=0}^{N-m}(-1)^{j}\left(\begin{array}{c}
M-m \\
j
\end{array}\right)(N-m-j)^{n}
$$

com a hipótese de que a chance de uma urna conter uma bola é a mesma para todas as urnas e todas as bolas.

Demonstração. O modelo probabilístico é o espaço de probabilidade finito equiprovável $\left(\Omega, 2^{\Omega}, P\right)$ cujo espaço amostral $\Omega$ é o conjunto de todas as sequências ordenadas de $n$ elementos $\left(z_{1}, z_{2}, \ldots, z_{n}\right)$ em que, para cada $j=$ $1,2, \ldots, n$ o elemento de número $j$ indica o número da urna que contém a bola número $j$.

Os eventos $A_{1}, A_{2}, \ldots, A_{N}$ são definidos por: para cada $k=1,2, \ldots, N$ o evento $A_{k} \subset \Omega$ é dado por

$$
A_{k}=\left\{\left(z_{1}, z_{2}, \ldots, z_{n}\right) \in \Omega: z_{1} \neq k, z_{2} \neq k, \ldots, z_{n} \neq k\right\}
$$

cuja probabilidade

$$
P\left(A_{k}\right)=\frac{(N-1)^{n}}{N^{n}}=\left(1-\frac{1}{N}\right)^{n}
$$

e a probabilidade

$$
P\left(A_{i} \cap A_{j}\right)=\frac{(N-2)^{n}}{N^{n}}=\left(1-\frac{2}{N}\right)^{n} i, j \in\{1,2, \ldots, N\}, \text { com } i \leq j
$$


além disso,

$P\left(A_{i} \cap A_{j} \cap A_{k}\right)=\frac{(N-3)^{n}}{N^{n}}=\left(1-\frac{3}{N}\right)^{n} i, j, k \in\{1,2, \ldots, N\}$, com $i \leq j \leq k$

o que mostra que os números $S_{k}^{(n)}$ do teorema de WARING são calculados como

$$
S_{k}^{(n)}=\left(\begin{array}{c}
N \\
k
\end{array}\right)\left(1-\frac{k}{N}\right)^{n} k=1,2, \ldots, N
$$

A probabilidade a ser calculada é a probabilidade de que exatos $m$ dos $N$ eventos $A_{1}, A_{2}, \ldots, A_{N}$ ocorrem, cujo valor é dado pelo teorema de Waring

$$
\begin{gathered}
\sum_{j=m}^{N}(-1)^{j-m}\left(\begin{array}{c}
j \\
m
\end{array}\right)\left(\begin{array}{c}
N \\
j
\end{array}\right)\left(1-\frac{j}{N}\right)^{n} \\
\left(\begin{array}{c}
N \\
m
\end{array}\right) \frac{1}{N^{n}} \sum_{j=0}^{N-m}(-1)^{j}\left(\begin{array}{c}
N-m \\
j
\end{array}\right)(N-m-j)^{n} .
\end{gathered}
$$

Uma aplicação do problema de ocupação de urnas é a questão de brindes em embalagens de produtos industrializados: se os brindes são rotulados com cartões numerados de 1 a $N$ de tal maneira que a chance de encontrar um cartão em uma embalagem é a mesma para todos os $N$ cartões. Na aquisição de $n$ embalagens, a probabilidade de não ocorrência de exatos $m$ dos cartões é então calculada como o problema de ocupação de urnas numeradas por bolas numeradas.

\subsection{LOTERIAS}

A loteria consiste na retirada de uma amostra não ordenada de $m$ bolas numeradas de uma urna contendo $n$ bolas numeradas com os dígitos $1,2,3, \ldots, n$, em que a chance de retirada de uma bola é a mesma e igual para todas as bolas da urna, com $m, n \in \mathbb{N}=\{1,2, \ldots\}$ e $m \leq n$.

O experimento aleatório da retirada de uma amostra não ordenada de $k$ números com $k \in\{1,2, \ldots, m\}$ em que todos as $n$ bolas numeradas tem chances iguais de serem sorteados, tem como modelo probabilístico o espaço finito equiprovável $(\Omega, \rho(\Omega), P)$ cujo espaço amostral $\Omega$ é o conjuto constituído de todas as $\left(\begin{array}{l}n \\ k\end{array}\right)$ subconjuntos com $k$ elementos do conjunto $\{1,2, \ldots, n\}$. 
A probabilidade de ocorrência de $k$ números previamente fixados é $\frac{\left(\begin{array}{l}k \\ k\end{array}\right)}{\left(\begin{array}{l}n \\ k\end{array}\right)}$.

A probabilidade de que desses $k$ números ocorram sem que nenhum deles sejam consecutivos, considerando 1 e $n$ consecutivos é dada por $\frac{\frac{n}{n-k} \cdot\left(\begin{array}{c}n-p \\ p\end{array}\right)}{\left(\begin{array}{l}n \\ p\end{array}\right)}$

Exemplo 1.24.1. Uma urna contém $2 n+1$ bolas numeradas de 1 a $2 n+1$, três dessas bolas são sorteadas aleatoriamente de uma só vez, sabendo que todas as bolas possuem a mesma chance de serem sorteadas, a probabilidade de que os números das três bolas sorteadas estejam em progressão aritmética (P.A) é dada por

$$
\frac{3 n}{4 n^{2}-1}
$$

O experimento aleatório consiste na retirada de uma amostra não ordenada de três bolas de uma urna que contém $2 n+1$ bolas numeradas de 1 até $2 n+1$, em que todas as bolas possuem a mesma chance de serem selecionadas.

O espaço de probabilidade correspondente é o espaço finito de probabilidade $\left(\Omega, 2^{\Omega}, P\right)$ cujo espaço amostral $\Omega$ é constituído pela totalidade dos $\left(\begin{array}{c}2 n+1 \\ 3\end{array}\right)$ subconjuntos de 3 elementos.

Para que as bolas sorteadas contenham números que estejam em P.A., os subconjuntos com 3 elementos têm a seguinte forma $\{x, x+r, x+2 r\}$ em que

$$
\begin{gathered}
r \geq 1,1 \leq x \leq 2 n-1 \text { e } x+2 r \leq 2 n+1 \\
1 \leq r \leq \frac{2 n+1-x}{2}
\end{gathered}
$$

para $x=1$,

$$
1 \leq r \leq \frac{2 n+1-1}{2} \text { ou } 1 \leq r \leq n
$$

para $x=2$,

$$
1 \leq r \leq \frac{2 n+1-2}{2}=n-\frac{1}{2}, \text { como r é inteiro ou } 1 \leq r \leq n-1
$$

para $x=3$,

$$
1 \leq r \leq \frac{2 n+1-3}{2} \text { ou } 1 \leq r \leq n-1
$$

Para $x=4$ e para $x=5$, implica $1 \leq r \leq n-2$ e assim por diante, até $x=2 n-2$ e $x=2 n-1$ implica $r=1$.

A probabilidade pedida é 


$$
\begin{aligned}
& \frac{n+2(n-1)+2(n-2)+\ldots+2(1)}{\left(\begin{array}{c}
2 n+1 \\
3
\end{array}\right)}= \\
& =\frac{n+2(n-1+n-2+\ldots+2+1)}{\frac{(2 n+1)(2 n)(2 n-1)}{3 !}} \\
& =\frac{n+2\left(\frac{(n-1)(1+n-1)}{2}\right)}{\frac{(2 n+1)(n)(2 n-1)}{3}} \\
& =\frac{n+(n-1) n}{\frac{(2 n+1)(n)(2 n-1)}{3}} \\
& =\frac{n^{2}}{n\left(4 n^{2}-1\right)} \\
& =\frac{3 n}{4 n^{2}-1}
\end{aligned}
$$

Exemplo 1.24.2. A Quina é uma loteria da Caixa Econômica Federal em que 5 números são sorteados entre os 80 disponíveis $(\Omega=\{1,2,3, \ldots, 80\})$, sabendo que todas os números possuem a mesma chance de serem sorteados, a probabilidade de que os 5 números sorteados formem uma progressão geomética (P.G.) é

$$
\frac{5}{\left(\begin{array}{c}
80 \\
5
\end{array}\right)}
$$

O experimento aleatório consiste na retirada de uma amostra não ordenada de 5 números de um conjunto que contém 80 números de 1 até 80 , em que todas os números possuem a mesma chance de serem selecionadas.

O espaço de probabilidade correspondente é o espaço finito de probabilidade $\left(\Omega, 2^{\Omega}, P\right)$ cujo espaço amostral $\Omega$ é constituído pela totalidade dos $\left(\begin{array}{c}80 \\ 5\end{array}\right)$ subconjuntos de 5 elementos.

Para que os 5 números sorteados formem uma P.G, os subconjuntos com 5 elementos têm a seguinte forma $\left\{x, x q, x q^{2}, x q^{3}, x q^{4}\right\}$, em que $x \in$ $\{1,2,3,4,5\}$ e $q=2$, pois para $q=1$ a P.G. ficaria constante e isso não seria possível, pois os números sorteados são distintos e para $q>2$ o maior elemento do subconjunto com 5 elementos ficaria maior que $80\left(x q^{4}>80\right)$

Como $q=2$ e $x \in\{1,2,3,4,5\}$, os possíveis resultados são 
$\{1,2,4,8,16\}$, para $x=1$;

$\{2,4,8,16,32\}$, para $x=2$;

$\{3,6,12,24,48\}$, para $x=3$;

$\{4,8,16,32,64\}$, para $x=4$;

$\{5,10,20,40,80\}$, para $x=5$.

A probabilidade procurada é $\frac{5}{\left(\begin{array}{c}80 \\ 5\end{array}\right)}$.

\subsubsection{MEGA-SENA}

A Mega-Sena é uma loteria, que paga o prêmio ao apostador que acertar os seis números sorteados em um conjunto de sessenta $(\Omega=\{1,2, \ldots, 60\})$. Também são pagos prêmios a quem acerta cinco números (quina) e quatro números (quadra). É permitido que o apostador faça a marcação de seis a quinze números dos sessenta possíveis em um mesmo cartão.

O experimento aleatório da retirada de uma amostra não ordenada de 6 números em que todos as 60 bolas numeradas tem chances iguais de serem sorteados, tem como modelo probabilístico o espaço finito equiprovável $\left(\Omega, 2^{\Omega}, P\right)$ cujo espaço amostral $\Omega$ é o conjuto constituído de todas as $\left(\begin{array}{c}60 \\ 6\end{array}\right)$ subconjuntos com 6 elementos do conjunto $\{1,2, \ldots, 60\}$.

a) A probabilidade de um apostador acertar a sena

i) com uma aposta simples (escolher 6 números do cartão):

$$
\frac{\left(\begin{array}{l}
6 \\
6
\end{array}\right)}{\left(\begin{array}{c}
60 \\
6
\end{array}\right)}=\frac{1}{50.063 .860} \cong 0,000002 \%
$$

ii) com uma aposta de sete números no cartão:

$$
\frac{\left(\begin{array}{l}
7 \\
6
\end{array}\right)}{\left(\begin{array}{c}
60 \\
6
\end{array}\right)}=\frac{7}{50.063 .860} \cong 0,000014 \%
$$

iii) com uma aposta de oito números no cartão:

$$
\frac{\left(\begin{array}{l}
8 \\
6
\end{array}\right)}{\left(\begin{array}{c}
60 \\
6
\end{array}\right)}=\frac{28}{50.063 .860} \cong 0,000056 \%
$$


iv) com aposta máxima de 15 números

$$
\frac{\left(\begin{array}{c}
15 \\
6
\end{array}\right)}{\left(\begin{array}{c}
60 \\
6
\end{array}\right)}=\frac{5005}{50.063 .860} \cong 0,01 \%
$$

b) A probabilidade do apostador acertar a quina

i) com uma aposta simples:

$$
\frac{\left(\begin{array}{c}
6 \\
5
\end{array}\right)\left(\begin{array}{c}
54 \\
1
\end{array}\right)}{\left(\begin{array}{c}
60 \\
6
\end{array}\right)}=\frac{324}{50.063 .860} \cong 0,00071 \%
$$

ii) com uma aposta de sete números no cartão:

$$
\frac{\left(\begin{array}{c}
7 \\
5
\end{array}\right)\left(\begin{array}{c}
53 \\
1
\end{array}\right)}{\left(\begin{array}{c}
60 \\
6
\end{array}\right)}=\frac{1113}{50.063 .860} \cong 0,00022 \%
$$

iii) com uma aposta de oito números no cartão:

$$
\frac{\left(\begin{array}{c}
8 \\
5
\end{array}\right)\left(\begin{array}{c}
52 \\
1
\end{array}\right)}{\left(\begin{array}{c}
60 \\
6
\end{array}\right)}=\frac{2912}{50.063 .860} \cong 0,0013 \%
$$

iv) com aposta máxima de 15 números

$$
\frac{\left(\begin{array}{c}
15 \\
5
\end{array}\right)\left(\begin{array}{c}
45 \\
1
\end{array}\right)}{\left(\begin{array}{c}
60 \\
6
\end{array}\right)}=\frac{135135}{50.063 .860} \cong 0,27 \%
$$

c) A probabilidade do apostador acertar a quadra é:

i) com uma aposta simples:

$$
\frac{\left(\begin{array}{c}
6 \\
4
\end{array}\right)\left(\begin{array}{c}
54 \\
2
\end{array}\right)}{\left(\begin{array}{c}
60 \\
6
\end{array}\right)}=\frac{21465}{50.063 .860} \cong 0,043 \%
$$

ii) com uma aposta de sete números no cartão:

$$
\frac{\left(\begin{array}{l}
7 \\
4
\end{array}\right)\left(\begin{array}{c}
53 \\
2
\end{array}\right)}{\left(\begin{array}{c}
60 \\
6
\end{array}\right)}=\frac{48230}{50.063 .860} \cong 0,096 \%
$$


iii) com uma aposta de oito números no cartão:

$$
\frac{\left(\begin{array}{l}
8 \\
4
\end{array}\right)\left(\begin{array}{c}
52 \\
2
\end{array}\right)}{\left(\begin{array}{c}
60 \\
6
\end{array}\right)}=\frac{92820}{50.063 .860} \cong 0,19 \%
$$

iv) com aposta máxima de 15 números

$$
\frac{\left(\begin{array}{c}
15 \\
4
\end{array}\right)\left(\begin{array}{c}
45 \\
2
\end{array}\right)}{\left(\begin{array}{c}
60 \\
6
\end{array}\right)}=\frac{135135}{50.063 .860} \cong 2,7 \%
$$




\section{Referências Bibliográficas}

[1] Magalhães, M.N. Probabilidade e Variáveis Aleatórias. 3. ed. São Paulo: Edusp, 2011.

[2] ROSS, S. Probabilidade: Um curso moderno com aplicações. 8 ed. Porto Alegre: Bookman, 2010.

[3] LIPSCHUTZ, S. Probabilidade. 4 ed. rev. São Paulo: Makron Books, 1993.

[4] WOODROOFE, M. Probability with applications, 1975.

[5] MORGADO, A.C. et al. Análise Cominatória e Probabilidade. 9 ed. Rio de Janeiro: SBM, 1991.

[6] FELLER, W.Introdução à teoria das probabilidades e suas aplicações. São Paulo: Edgard Blucher Ltda, 1976.

[7] HAZZAN, S. Matemática Elementar. São Paulo: Atual, 1977.

[8] MEYER, P.L.Probabilidade Aplicações á Estatística. 2 ed. Rio de Janeiro: Livros Técnicos e Científicos, 2009.

[9] LIMA, E.L. et al. A Matemática do Ensino Médio. v 4 Rio de Janeiro: SBM, 2010.

[10] PARZEN, E. Modern Probability Theory and Its Applications. Witey Classes Library Edition, 1992. 